Friedrich-Schiller-Universität Jena

Fakultät für Sozial- und Verhaltenswissenschaften

Institut für Soziologie

Lehrstuhl für allgemeine und theoretische Soziologie

\title{
LIFELOGGING AUF INSTAGRAM
}

\section{Digitale Selbstvermessung und -darstellung zwischen disruptiver Technologie und kulturellem Wandel}

\author{
Masterarbeit zur Erlangung des akademischen Grades \\ Master of Arts (M.A.) \\ Vorgelegt von Magdalena Riedl \\ Matrikelnummer: 180416 \\ Note: 1,0 \\ geboren am 21.06.1995 in Garmisch-Partenkirchen
}

Erstgutachterin: Dr. Diana Lindner

Zweitgutachter: Dr. Jörg Oberthür

Jena, 20.10.2020 


\begin{abstract}
Vor dem Hintergrund sozialer Beschleunigung, Datafizierung und Ökonomisierung lässt sich in der Moderne eine Loslösung traditioneller Bindungen und ein Wandeln moderner Kerninstitutionen beobachten. Für die Akteure bedeuten diese Dynamiken auf der einen Seite gesteigerte Handlungsmöglichkeiten und -spielräume aber auf der anderen Seite auch der Wunsch nach Ordnung und Kontingenzreduktion. In einem auf immaterielle Ressourcen ausgerichteten Kapitalismus werden dabei verschiedene Aspekte des Körpers zunehmend relevant für ökonomische Wertschöpfungsketten. Stefan Selke beschreibt in diesem Zusammenhang mit dem Begriff des „Lifelogging“ die digitale Erfassung des Lebens in Echtzeit und einer dadurch ausgelösten Verschiebung des sozialen Blicks auf den Menschen. Als ausschlaggebend für diese Entwicklung wird die zunehmende Quantifizierung betrachtet. Auch das Projekt „Das vermessene Leben“ beschäftigt sich mit den ambivalenten Folgen einer in hohem Maße auf quantitative Steigerung ausgerichteten Optimierungslogik, wie sie im Zuge des digitalen Wandels an Bedeutung gewonnen hat. Dabei ist noch nicht geklärt, worin der kategoriale Unterschied qualitativer und quantitativer Selbstthematisierung besteht und worin die vermeintliche Besonderheit quantitativer Selbstvermessung liegt. An dieser Stelle knüpft die Arbeit an, indem sie den Erkenntnissen aus der Forschung zu quantitativer Selbstvermessung eine empirische Untersuchung qualitativer Selbstthematisierung gegenübergestellt und durch eine Analyse der digitalen Selbstdarstellung auf Instagram zeigt, dass nicht die Quantifizierung, sondern vielmehr der auf Dauer gestellte (öffentliche) Vergleich und die Bewertung der Subjekte entscheidend ist. Die vorgenommene zweiteilige Untersuchung forscht nach Unterschieden und Gemeinsamkeiten quantitativer und qualitativer Selbstthematisierung und stellt die in der Literatur konstatierte „Herrschaft der Zahl" in Frage.
\end{abstract}




\section{Inhaltsverzeichnis}

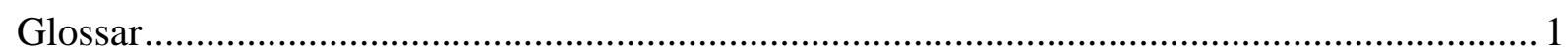

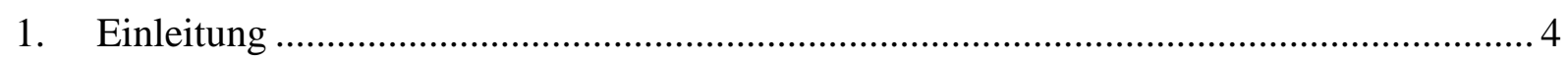

2. Dynamische Stabilisierung und die Modernität des Vergleichs ........................................ 9

2.1. Dynamische Stabilisierung im Kontext der Digitalisierung ........................................ 9

2.2. Vergleichen und Bewerten in der Moderne .............................................................. 13

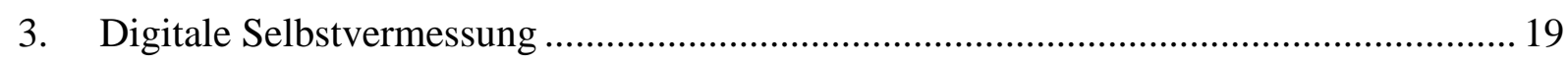

3.1. Bedeutungsproduktion durch digitale Selbstvermessung ........................................ 19

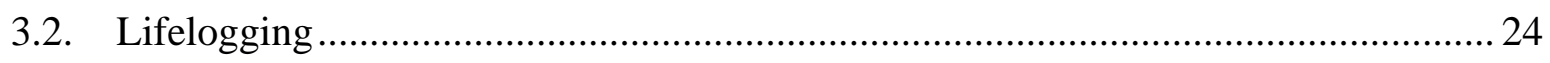

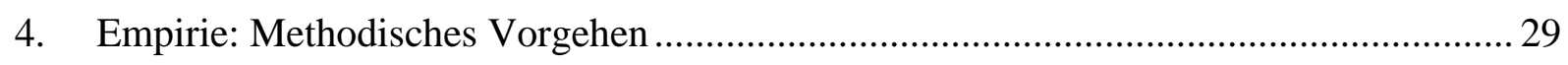

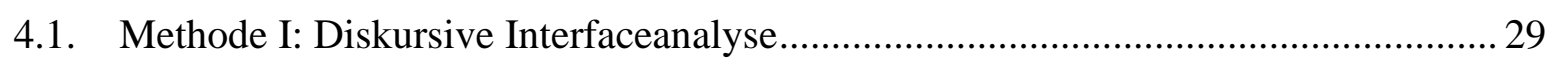

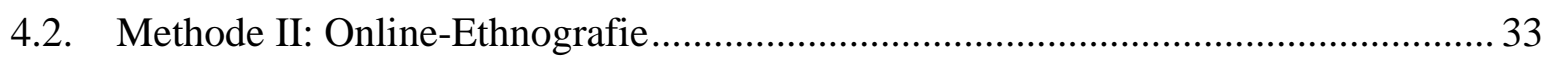

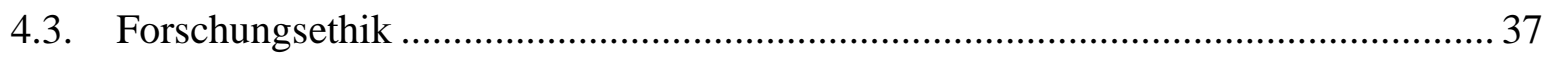

5. Digitale Selbstdarstellung auf Instagram ……………………..................................... 40

5.1. Beschreibung des Untersuchungsgegenstandes ....................................................... 40

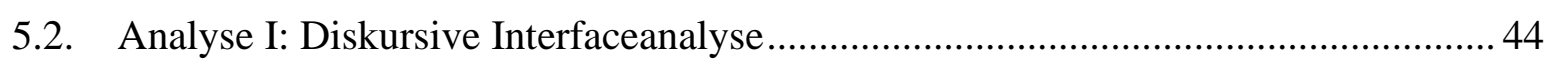

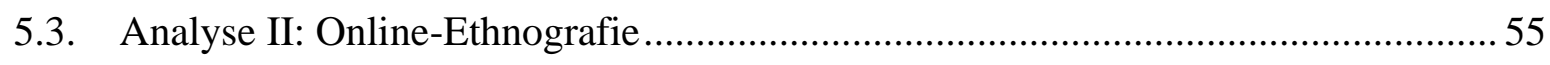

5.4. Vergleichen und Bewerten auf Instagram................................................................ 76

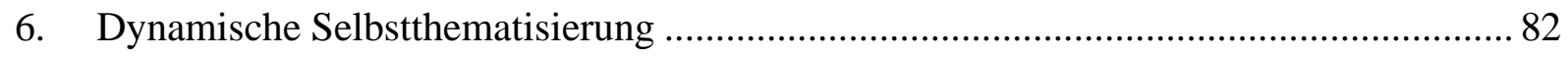

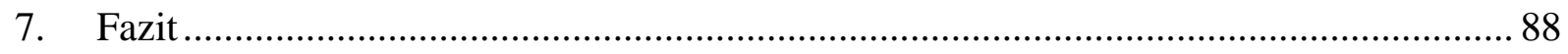

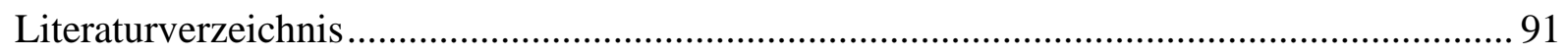

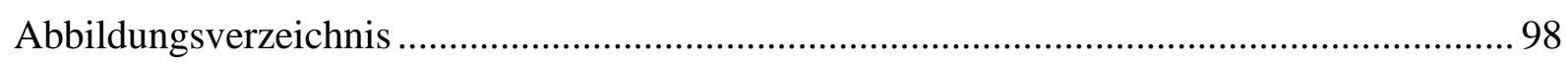

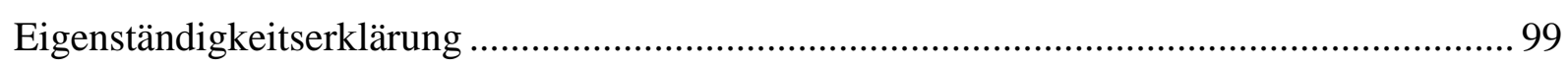




\section{Glossar}

Zur besseren Verständlichkeit von Begriffen, die vor allem im empirischen Teil der Arbeit relevant werden, ist hier ein Glossar eingefügt. Zudem sind die erklärten Begriffe bei der ersten Nennung im Fließtext unterstrichen.

Bio Kurzbeschreibung des Accounts. In der Profilansicht unterhalb der Followeranzahl und rechts neben dem Profilbild angesiedelt.

Boomerang Kurze Videosequenzen, die sich wie ein Bumerang vor und zurück bewegen. Meist bestehen die Videos aus nur 10 Einzelaufnahmen, die wiederholt abgespielt werden. Es handelt sich dabei also um sehr kurze Videosequenzen, die wiederholt abwechselnd vorwärts und rückwärts abgespielt werden.

Content Ist die englische Bezeichnung für Inhalt. Er umfasst auf einer Website und in sozialen Medien alle Bestandteile, die Informationen und Bedeutung tragen. Neben den Texten gehören beispielsweise Bilder, Grafiken, Audiodateien, Videos zum Content.

Community Mit einer (Online-)Community wird eine mehr oder weniger organisierte Gruppe von Menschen bezeichnet, die im Internet miteinander kommunizieren und im virtuellen Raum miteinander interagieren. Durch das Folgen anderer Profile entsteht ein abgrenzbares soziales Netzwerk von Nutzer*innen mit von ihnen erzeugten Inhalten.

DM Ist eine private Nachricht an einen User, die nicht in der Timeline auftaucht, sondern in einem speziellen, nicht-öffentlichen Postfach landet, und ist die Abkürzung für Direct Message.

Emoji (oder Ein Smiley ist die grafische Darstellung eines Gesichtsausdruckes, die oft Smiley) verwendet wird, um eine bestimmte Emotion wiederzugeben oder sie zu verdeutlichen. Der Begriff wird oft synonym zu Emoji verwendet. In Instagram kann über die Smartphone-Tastatur eine Vielzahl solcher Symbole aufgerufen werden.

\section{Engagement-} rate

Die Engagementrate misst die Interaktion auf Plattformen wie Instagram, oder Facebook. Sie wird aus der durchschnittlichen Summe von Likes, Kommentaren und Shares pro Tag geteilt durch die Gesamtzahl der Follower berechnet. 
Feed

GIF

Influencer

NikeFuels

Timeline

Regler/ EmojiSlider
Im Feed finden sich nacheinander aufgelistet, die verschiedenen neuen Posts. Auf dem Startbildschirm der App kann man durch die - nach Relevanz sortierten - neuen Bilder und Videos abonnierter Profile scrollen. In der Suchfunktion sieht man im sogenannten Entdecker-Feed neuen Content nicht abonnierter Profile (Als relevant werden Posts von Profilen eingestuft, mit denen man in Form von Likes, Kommentaren o.ä. interagiert oder nach denen man gezielt sucht).

Ein GIF steht für Graphics Interchange Format ist ein spezielles Grafikformat, ähnlich wie ein JPG. Eine GIF-Datei ist häufig animiert. Meistens wiederholt sich die Animation nach wenigen Sekunden. Oft werden darin Ausschnitte aus Serien, Filmen oder von berühmten Personen abgebildet.

Als Influencer werden seit den 2000er Jahren Personen bezeichnet, die aufgrund ihrer starken Präsenz und ihres hohen Ansehens in sozialen Netzwerken als Träger für Werbung und Vermarktung infrage kommen.

Bei dem sogenannten NikeFuel handelt es sich um eine von der Marke Nike entwickelte „Währung“, die verspricht, sämtliche sportliche „Leistungen" in Punkte umzurechnen und so verschiedene Sportarten untereinander vergleichbar zu machen. https://www.fitforfun.de/sport/fitnessstudio/nike-fuel-das-training-der-zukunft_aid_12617.html (zuletzt aufgerufen am 04.09.2020).

Mit der Timeline wird die in der Regel chronologische Auflistung von Posts bezeichnet. Timeline und Feed wird in dieser Arbeit synonym verwendet.

Ist eine simple Variante für mehr Interaktion in den Stories und dient als standardisiertes Bewertungstool. Eingefügt in die Story kann der oder die Betrachter*in den Regler vollständig oder teilweise nach rechts wischen. Voreingestellt ist der Emoji mit Herzaugen, theoretisch kann aber jedes Emoji eingestellt werden und so die Bewertung verschiedener Gefühlszustände abgefragt werden.

\section{|Ask a question...}

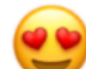

Abbildung 1: Emoji-Slider 
Story und Instagram Stories ähneln der bekannten Snapchat-Funktion, auf welcher Story-Repost eingestellte Fotos und Videos nach 24 Stunden automatisch verschwinden. Innerhalb der Stories können neben Bildern und Videos auch Boomerangs oder Textelemente enthalten sein. Für Instagram-Stories gibt es dabei keine direkte Kommentar- oder Likefunktion, dafür allerdings die Möglichkeit, eine DM zu versenden, oder durch eine Quick Reaction (siehe. S. 49 in dieser Arbeit) zu reagieren.

Wird man in einer Story markiert, das heißt, das eigene Profil mit der geposteten Story verknüpft, hat man die Möglichkeit, diese fremde Story in der eigenen Story zu posten. Bei diesem Repost können der ursprünglichen Story noch weitere Gestaltungselemente wie Text, oder Bilder hinzugefügt werden. Diese Funktion ermöglicht damit eine öffentlich sichtbare Kommunikation zwischen Profilen. 


\section{Einleitung}

Was haben Selfies auf sozialen Netzwerken mit Fitness-Apps gemeinsam? Auf den ersten Blick vermutlich nicht viel - das eine ein fotografischer, im besten Fall ästhetischer, Schnapsschuss aus dem Alltag, das andere eine wissenschaftlich anmutende, zahlenförmige Aufzeichnung der sportlichen Leistung. Bei weiteren Überlegungen jedoch erschließt sich beides als eine Möglichkeit, das eigene Selbst zum Thema zu machen und für andere sichtbar abzubilden. Während das Selfie eine qualitative Möglichkeit bietet, das Selbst darzustellen, dient die Fitness-App zur quantitativen Sichtbarmachung desselben. Außerdem bedarf es in beiden Fällen technischer Geräte, ohne die das Selfie oder das Tracken der Laufstrecke nicht möglich wären. Die technische und eigenständige Erfassung des Selbst setzt dabei eine Entwicklung fort, die Michel Foucault schon Mitte der 1970er Jahre beschrieben hat. In „Überwachen und Strafen“ verhandelt er die immer feinteiligere Erfassung und Nutzbarmachung des Körpers. Die Disziplinierung des Menschen als neues Machtinstrument ist dabei die zentrale These und zeigt, wie sich anfangs im Militär, aber auch in Schulen und dann in immer mehr Bereichen die Inkorporation von Zwängen ausdehnt, die die Subjekte einer zunehmenden Disziplin unterwerfen. Muss diese zunächst noch von außen überwacht werden, verinnerlichen die Subjekte in der ,panoptischen“ Gesellschaft das, was Foucault einen ,,potentiellen Überwacher“ nennt (Foucault 1993).

Die aktuell reichlich diskutierte These lautet, dass die meist technisch vermittelte und detaillierte Erfassung des Körpers eine immer präzisere Disziplinierung und Optimierung ermöglicht. Im Zentrum dieser Überlegungen stehen dabei überwiegend quantitative Formen der Selbstvermessung. So beschäftigen sich beispielsweise Lisa Wiedemann oder Uwe Vormbusch und Karolin Kappler mit Selbstvermessung im Kontext der von Foucault beschriebene Disziplinargesellschaft (Vormbusch \& Kappler 2016; Wiedemann 2016). Auch Stefan Selke beschäftigt sich mit der metrischen Protokollierung des eigenen Lebens. Er geht dabei davon aus, dass die Verbreitung von Lifelogging, also die Übersetzung des Lebens in Daten, einhergeht mit neuen sozialen Kategorisierungen, die unter anderem mit einer verstärkten horizontalen Kontrolle verbunden sind. Im Anschluss daran fragt diese Arbeit danach wie Subjektivierung durch digitale Technologien funktioniert, welche Normen und Werte durch die Technik an die Menschen herangetragen werden, aber auch wie sich die Subjektivierungsweise gestaltet und welches Widerständigkeitspotenzial sich identifizieren lässt.

Den sozialtheoretischen Hintergrund bilden dabei verschiedene Theorien der Moderne, von Rosas Theorie sozialer Beschleunigung (Rosa 2016a) über die Verflüssigung der Moderne 
(Bauman \& Jakubzik 2007) und verschiedene Erschöpfungsdiagnosen (Neckel 2005). Zusammenfassend stehen Prozesse der Beschleunigung, Individualisierung, Vernetzung, Ökonomisierung und Datafizierung und dabei immer auch Disziplinierung im Foucualt'schen Sinne im Fokus. Im Zuge dieser übergreifenden Dynamiken lässt sich eine Loslösung aus traditionellen Bindungen ebenso beobachten wie ein Wandel der drei modernen Kerninstitutionen Arbeitsmarkt, Sozialstaat und Familie. Die bisher scheinbar festgefügte Institutionenstruktur westlicher Industriegesellschaften weist immer mehr Reibungspunkte und Diffusionspotenzial auf: So kommt es zu einem Verschwimmen bislang klarer Grenzziehungen zwischen den drei Institutionen und auch die an sie gekoppelten Logiken verändern sich. ${ }^{1}$ Für die Akteure bedeutet dies auf der einen Seite gesteigerte Handlungsmöglichkeiten und -spielräume, aber auf der anderen Seite auch den Wunsch nach Ordnung und Kontingenzreduktion. In diesem Kontext lässt sich der bereits beschriebene Trend einordnen, dass sich Menschen mithilfe technischer Geräte selbst vermessen. Verschiedene Aspekte, wie der Herzrhythmus, die Schrittzahl, Schlafphasen aber auch Kalorienzufuhr oder die eigenen Gefühle, werden dabei in meist quantitative Daten übersetzt und so zugänglich für Kontroll- und Optimierungsansprüche. Diese Entwicklung, die beispielsweise auch von der sogenannten „Quantified Self“-Bewegung getragen wird, steht im Mittelpunkt der Forschung von Vormbusch und Selke, verliert dabei aber aus dem Blick, dass auch abseits quantitativer Daten immer mehr Facetten des menschlichen Lebens in (qualitative) Daten übersetzt werden, Selfies auf sozialen Netzwerken sind dafür nur ein Beispiel.

An dieser Stelle möchte die vorliegende Arbeit ansetzen und Stefan Selkes Überlegungen zu Lifelogging und der digitalen Selbstvermessung systematisch durch die digitale Selbstdarstellung auf Instagram erweitern und dabei die von Selke implizit konstatierte Besonderheit der Zahl kritisch hinterfragen. Auch wenn Stefan Selke in seiner Definition zu Lifelogging die Übersetzung des Lebens in Daten allgemein beschreibt, fokussiert er sich in seinen theoretischen Überlegungen auf Selbstvermessungstechnologien, die das Selbst in lediglich numerische Objekte übersetzen. Die Arbeit setzt am heuristischen Begriff des Lifelogging an und nimmt auch qualitative Formen digitaler Selbstdarstellung ernst. Mit dieser offenen Perspektive ist es möglich, nach einem kategorialen Unterschied qualitativer und quantitativer Wirklichkeitskonstruktion durch Technologie zu forschen.

\footnotetext{
${ }^{1}$ Klaus Dörre spricht beispielsweise in diesem Zusammenhang von einer zunehmenden Prekarisierung der Arbeit und einer Erosion der Normalarbeiterverhältnisse (Dörre 2010). An anderer Stelle wird von einer Subjektivierung der Arbeit gesprochen, die immer mehr Aspekte des privaten Selbst in den Bereich der Erwerbsarbeit transformiert (Kleemann 2012). Spiegelbildlich dazu konstatiert Ulrich Bröckling die Ökonomisierung des Sozialen (Bröckling 2013).
} 


\section{Forschungsinteresse und Aufbau der Arbeit}

Ausgangspunkt für diese Überlegungen war der Expert*innen-Workshop im Projekt „Das ver-

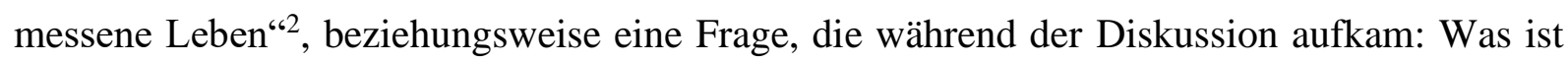
der kategoriale Unterschied, der qualitative und quantitative Wirklichkeitskonstruktion voneinander abgrenzt? Diese Frage entstand am Rande der Fallbetrachtung eines Interviews, das im Rahmen des Projekts durchgeführt wurde. Gemeinsam mit Steffen Krüger, Uwe Vormbusch und Birgit Blättel-Mink als Expert*innen und den verschiedenen wissenschaftlichen Mitarbeiter*innen wurden im Februar 2020 Expert*inneninterviews aus dem Jenaer Teilprojekt in Bezug auf Quantifizierungsprozesse hin befragt, wobei Steffen Krüger sich an einer Stelle die oben genannte Frage stellte. Betrachtet man Forschungen rund um das vermessene Leben, scheint außer Frage zu stehen, dass wir uns in einem Prozess erneuter Rationalisierung und Quantifizierung befinden. Dabei stehen verschiedene Subjektformierungen im Mittelpunkt, die aus dem Subjekt ein formbares, vermessbares und kalkulierbares machen (Krämer, Franz \& Klinge 2019). Immer mehr Bereiche der subjektiven Welt werden dabei für die quantitative Verdatung zugänglich gemacht und schon lange werden nicht mehr lediglich Schrittzahlen erfasst, sondern auch der Schlaf, das Essverhalten, hormonelle Schwankungen und Gefühle. Das Subjekt folgt damit den beschriebenen Dynamiken der Beschleunigung und den Forderungen eines Kapitalismus, der infolge neoliberaler Anpassungen auf die Eigeninitiative und Eigenverantwortung der Akteure setzt (Schaupp 2016; Stark 2016). Für diese „Erfolgsgeschichte“ ist nicht nur eine immer feinere Mikrophysik der Macht entscheidend, sondern eben auch die Verlagerung von Kontrolle und Disziplin in die Subjekte selbst. Wieso sich die Praktiken der Selbstvermesser*innen, aber auch die Selbstdarstellung auf Instagram und anderen Plattformen, sogar als noch effizientere Kontrollinstrumente als das von Jeremy Bentham entworfene Panopticon erweisen können, wird sich später zeigen (Kapitel 6).

Durch neue Formen der Selbstthematisierung verändert sich die Sorge um sich selbst. Was die Arbeiten von Jörg Strübing, Uwe Vormbusch oder Stefan Selke bei der Untersuchung dieses Sachverhalts allerdings nicht leisten, ist eine trennscharfe Herausarbeitung der quantitativen Eigenheiten solcher Praktiken (Duttweiler et al. 2016; Selke 2016a; Vormbusch 2012). Zudem

\footnotetext{
${ }^{2}$ Das transdisziplinäre Projekt „,Das vermessene Leben. Produktive und kontraproduktive Folgen der Quantifizierung in der digital optimierenden Gesellschaft“ wird geleitet von Vera King (Sigmund-Freud-Institut \& Goethe Univ., Projektsprecherin;), Benigna Gerisch (International Psychoanalytic Univ. Berlin) und Hartmut Rosa (Univ. Jena \& Max-Weber-Kolleg Erfurt), gefördert von der VolkswagenStiftung in der Förderlinie „Schlüsselthemen für Wissenschaft und Gesellschaft“", Projektbeginn in 2/2018; wissenschaftliche Mitarbeiter*innen des Projekts an der IPU Berlin, an Goethe-Univ. \& SFI Frankfurt sowie der Univ. Jena sind: Charlotte Findeis, Diana Lindner, Benedikt Salfeld, Micha Schlichting, Julia Schreiber, Maike Stenger, Stella Voigt.
} 
gruppieren sich die Vorreiter der „Quantifed Self“-Bewegung ${ }^{3}$ noch um einen relativ kleinen Kreis an Menschen, bei denen vor allem gesundheitliche Prävention und Optimierung oder die Erfassung der Gefühle im Vordergrund stehen. Zwar hält vor allem die Aufzeichnung sportlicher Leistungen in Form von verschiedenen Smartphone-Apps wie Runtastic, Fitbit oder Freeletics Einzug in den Alltag von immer mehr Menschen, festen Bestandteil bilden diese Praktiken allerdings noch nicht in einer breiten Masse der Bevölkerung. ${ }^{4}$

Viel weiter verbreitet und tiefer im Alltag verankert sind dagegen Social Media Apps wie Instagram, oder TikTok die vor allem von jüngeren Nutzer*innen frequentiert werden. So nutzen 98 \% der 14-29-Jährigen WhatsApp und Instagram zählt nach Facebook zu den beliebtesten Apps (59 \% der 14-29-Jährigen). ${ }^{5}$ Auf Instagram können Menschen vor allem Fotos und Videos, aber auch schriftlichen Content mit Followern teilen, mithilfe von Hashtags einer größeren Öffentlichkeit zugänglich machen oder nur temporär sichtbar mit einem kleinen Kreis an „engen Freunden“ teilen. Dabei weist die Selbstdarstellung auf Instagram trotz ihres Fokus auf Bilder und Videos - so die These - hohe Ähnlichkeiten zu Selbstvermessungspraktiken auf und zieht ähnliche Folgen für die Subjekte nach sich. Bei der Erforschung des sich selbst vermessenden Subjekts schließt sich zwangsläufig die Frage nach seiner Darstellung in der sozialen Welt an. Diese Frage wird allerdings von den bisherigen Arbeiten nicht oder nur unzureichend behandelt. Das Forschungsinteresse dieser Arbeit richtet sich deshalb explizit auf eine vergleichende Betrachtung qualitativer und quantitativer Formen der Selbstthematisierung und möchte über die Erforschung von Nutzer*innen auf Instagram eine kontrastierende Betrachtung ermöglichen. Der Analyse unterliegt die Annahme, dass sowohl digitale Selbstdarstellung als auch Selbstvermessung Orte des Vergleichens und Bewertens bilden. Im Zentrum der empirischen Analyse stehen demnach Vergleichs- und Bewertungspraktiken auf Instagram, um in der Synthese eine gemeinsame Betrachtung von quantitativer und qualitativer Selbstbeschreibung zu ermöglichen.

Die Forschungsfrage der Arbeit lässt sich also folgendermaßen formulieren: Worin unterscheiden sich qualitative und quantitative Formen der digitalen Selbstthematisierung und wie ist diese Differenzierung zu bewerten?

\footnotetext{
${ }^{3}$ https://quantifiedself.com/ (zuletzt aufgerufen am 04.09.2020).

${ }^{4}$ Laut einer Online-Umfrage nutzt derzeit rund ein Viertel der Menschen in Deutschland Fitnesstracker https://www.splendid-research.com/de/statistiken/item/studie-einstellung-deutsche-tracking.html (zuletzt aufgerufen am 15.09.2020), während fast die Hälfte der Deutschen zu den aktiven Social-Media-Nutzenden zählt https://www.dwif.de/news-events/zahl-der-woche/kpi/item/social-media-nutzung-tourismus.html (zuletzt aufgerufen am 15.09.2020).

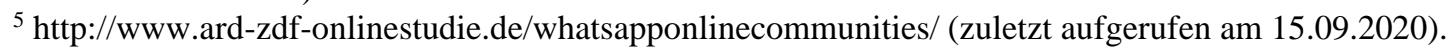


Es wird geprüft, ob sich Effekte, die auf die Quantifizierung zurückgeführt werden, vielmehr aus der Ausdehnung des Vergleichshorizonts und einer Veränderung von Vergleichspraktiken ergeben. Eine zentrale These, die dabei erarbeitet wird, ist, dass die Effekte und Folgen, die sich aus der Selbstvermessung (und -darstellung) ergeben, nicht allein auf ihren quantitativen Charakter allein zurückzuführen sind. Als Alternative wird vorgeschlagen, in der Analyse den Vergleich bzw. die Vergleichbarmachung als relevante Dimension stärker zu berücksichtigen.

Im Anschluss an bestehende Arbeiten zu digitaler Selbstvermessung im Allgemeinen und Lifelogging im Speziellen steht im Zentrum der Arbeit eine empirische Untersuchung der Selbstdarstellung auf Instagram. In einem ersten Schritt werden in der App verankerte Normen und Werte mithilfe einer diskursiven Interfaceanalyse herausgearbeitet. Damit wird nach der Subjektformierung auf Instagram gefragt, oder, mit anderen Worten, danach, welches Bild eines/r ,perfekten“ Nutzers oder Nutzerin in das Interface eingebettet ist und welche Handlungsoptionen den Subjekten vom Interface der App nahegelegt werden. Die konkrete Subjektivierungsweise wird in einer zweiten Teiluntersuchung mithilfe eines ethnografischen Ansatzes untersucht. Durch Beobachtungen und Interaktion mit Instagram-Userinnen wird dabei nach konkreten Handlungspraktiken geforscht.

Die Arbeit gliedert sich dabei in vier Teile: Im folgenden Kapitel schließt sich zunächst eine genauere Betrachtung des sozialtheoretischen Hintergrunds der Arbeit und die Beschreibung grundlegender Dynamiken der westlichen Moderne an. Dabei wird Hartmut Rosas Konzept der dynamischen Stabilisierung als wichtiger Motor der Moderne skizziert, um daraus die Folgen des kulturellen Wandels für die Subjekte verständlich zu machen. Daraufhin wird eine Zunahme an Vergleichs- und Bewertungspraktiken als Reaktion auf die Beschleunigung und Kontingenzsteigerung vorgestellt, welche in der späteren Analyse eine zentrale Bedeutung zukommt (Kapitel 2). Im zweiten Teil wird ein Überblick über die bestehende Literatur zu digitaler Selbstvermessung gegeben und Stefan Selkes Konzept des Lifelogging vorgestellt (Kapitel 3). Danach wird im dritten Teil die eigene empirische Untersuchung vorgestellt (Kapitel 4 und 5). Dieser Abschnitt gliedert sich in die Vorstellung der Methode, forschungsethische Überlegungen, die Vorstellung der Ergebnisse aus beiden Teiluntersuchungen sowie einer abschlieBenden Interpretation. Im letzten Teil werden in Kapitel 6 Überlegungen zu quantitativer und qualitativer Selbstthematisierung zusammengeführt und die Forschungsfrage in Kapitel 7 beantwortet. 


\section{Dynamische Stabilisierung und die Modernität des Vergleichs}

Beschleunigung, Individualisierung, Vernetzung, Ökonomisierung und Datafizierung - das alles sind Prozesse, die der Moderne zugeschrieben werden. Die existierende Literatur und Theoriebildung ist dabei vielfältig und fokussiert jeweils andere Aspekte der Moderne. Diese Arbeit bezieht sich vor allem auf die Theorie sozialer Beschleunigung von Hartmut Rosa, da diese in der Lage ist, diese vielfältigen Begriffe unter dem Modus der Dynamisierung zu beschreiben (Rosa 2016a).

\subsection{Dynamische Stabilisierung im Kontext der Digitalisierung}

Insgesamt lässt sich, wie bereits eingangs erwähnt, eine Loslösung aus traditionellen Bindungen ebenso beobachten wie ein Wandel der modernen Kerninstitutionen, welche für die Moderne charakteristisch sind. In all diesen Institutionen erkennt Rosa eine Dynamisierung materieller, geistiger und sozialer Prozesse. Kennzeichen der Moderne ist aber nicht dieser Prozess für sich genommen, sondern die Tatsache, dass die Dynamisierung selbst zum strukturellen Zwang geworden ist. Das System erhält sich in und durch die als Steigerung zu verstehende Bewegung. Zeitlich, sachlich und sozial ist die Moderne in nahezu allen Bereichen auf systematisches Wachstum angewiesen. Rosa entwirft für dieses Phänomen den Begriff der dynamischen Stabilisierung, der eben diese paradox anmutende Stabilisierung durch Wachstum begrifflich fassbar macht (vgl. Rosa 2016b, 676). ${ }^{6}$ Die Moderne zeichnet sich demnach dadurch aus, dass für die Reproduktion des Bestehenden eine systematische Steigerung in den Bereichen (ökonomisches) Wachstum, Beschleunigung und Innovationsrate strukturell notwendig ist. Empirisch

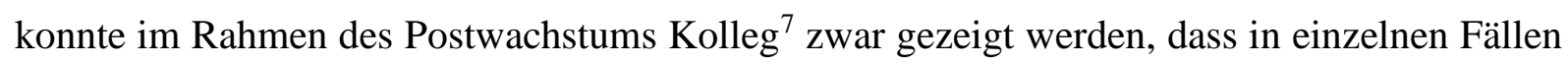
auch die Stagnation oder der Rückgang von Wachstum sich nicht notwendigerweise zu systemrelevanten Krisen ausweiten muss (Bsp. Wirtschaft in Japan, Griechenland), die grundlegende Bedeutung der Stabilisierung durch Wachstum bleibt aber dennoch bestehen (wenn auch nicht als alleiniges Strukturprinzip der Moderne). Hartmut Rosa deutet diese empirischen Prozesse als Offenbarung von Desynchronisationsprozessen. Daran geknüpft ist seine Beobachtung, dass sich nicht alle gesellschaftlichen Sphären als gleichermaßen beschleunigungsfähig, bzw. -willig erweisen. Die daraus entstehenden Desynchronisationsprozesse und -krisen lassen sich nach Rosa als temporalspezifische Überforderung ,langsamer“ Systeme beschreiben (vgl. Dörre \& Rosa 2015).

\footnotetext{
${ }^{6}$ Wahlverwandt zum Begriff der dynamischen Stabilisierung ist der von Paul Virilio geprägte Begriff des rasenden Stillstands (siehe Virilio 2015).

${ }^{7}$ Zur Homepage des Kollegs: http://www.kolleg-postwachstum.de/ (zuletzt aufgerufen 08.10.2020).
} 
Für die Arbeit besonders spannend in Rosas Theorie ist die Annahme, der Steigerungsimperativ sei nicht nur auf Makroebene bedeutsam, sondern reiche bis in die alltägliche Lebensführung der Menschen. Dies zeigt sich vor allem in Normen und Werten, besonders deutlich aber in der biografischen Verortung (vgl. Rosa 2016b, 684). Sinnbildlich dafür steht Ulrich Bröcklings Idealtyp des Unternehmerischen Selbst (Bröckling 2013), bei dem das Subjekt ${ }^{8}$ die Zwänge des Marktes zunehmend verinnerlicht und lernt, sein Leben als erfolgreiches Projekt zu gestalten. Dabei bildet der Idealtyp des Unternehmerischen Selbst kein Werkzeug zur Beschreibung der Wirklichkeit, sondern ein Instrument, um sie zu verändern (vgl. ebd., 48). ${ }^{9}$ Das Konzept setzt damit auf der Ebene der Subjektformierung an und trifft Aussagen über idealtypische Eigenschaften, welche an die Subjekte herangetragen werden. Von ihnen wird eine erweiterte Selbstorganisation und -kontrolle sowie ein Zwang zur Ökonomisierung und Kapitalisierung der alltäglichen Lebensführung gefordert, wobei dieser Ökonomisierungsdruck alle Bereiche des Alltags erfasst. Folgt man den Überlegungen Bröcklings, prägen die Maxime unternehmerischen Handelns das Verhältnis der Individuen zu sich selbst und zu anderen und diffundieren in die unterschiedlichsten Bereiche des Sozialen (vgl. ebd., 50). Sehr passend erscheint an dieser Stelle die Metapher des Marktplatzes des Lebens, auf dem Exzellenz und Wettbewerb als handlungsleitende Werte ökonomischen Erfolg und Selbstverwirklichung versprechen. Die Idee, das Leben als Unternehmen zu gestalten, impliziert dabei, dass man durch das ganze Leben hinweg beschäftigt bleibt, wenn auch nur in diesem einen ,eigenen“ Unternehmen (vgl. ebd.).

Damit verbunden ist die Vorstellung einer ständigen Geschäftigkeit, wenn es darum geht, eine angemessene Rendite aus der Reproduktion, Rekonstruktion und Erhaltung des eigenen $\mathrm{Hu}$ mankapitals zu erzeugen. Das entspricht der Sorge um sich, wie sie Foucault bereits in seinen Governmentality Studies beschreibt (vgl. Gordon 2009). Das Leitbild des Unternehmerischen Selbst wird internalisiert und die Mikrophysik der Macht wird damit zunehmend feiner. So sind nichtmehr nur das Militär, die Schule oder die Arbeit Schauplatz der von Foucault so treffend

\footnotetext{
${ }^{8}$ In dieser Arbeit wird das Subjekt im Sinne Foucaults als produktives verstanden, das immer in Machtverhältnisse eingebettet ist. Dabei ist dem Subjekt in dieser Denkrichtung eine zweifache Bedeutung immanent: Ein Subjekt zu sein heißt, jemandem unterworfen zu sein, aber auch, durch Selbsterkenntnis der eigenen Identität verhaftet sein. Foucault untersucht in diesem Kontext Machtformen, die aus einem Individuum ein Subjekt machen, indem sie das Individuum in Kategorien unterteilen und ihm eine spezifische Individualität aufprägen. Das moderne Subjekt findet sich dabei einer gleichermaßen totalisierenden wie individualisierenden Form der Macht unterworfen, deren neues Ziel die Selbstverwirklichung ist (Foucault 1994).

${ }^{9}$ Mit der Rahmung des Unternehmerischen Selbst als Idealtyp wird eine zwingende Differenz zwischen theoretischem Konzept und Realität deutlich, da der Idealtyp nach Weber ein Instrument zur Beschreibung der Wirklichkeit darstellt, das vor allem das Ziel verfolgt zugrunde liegenden Strukturen und Merkmale herauszuarbeiten, bleibt er als Deskription unvollständig (vgl. Weber 2018) Wird also hier von einem Werkzeug zur Beschreibung der Wirklichkeit gesprochen, knüpft das an die durch Weber geprägte Definition eines Idealtyps an.
} 
beschriebenen Disziplinierung, sondern auch das Privatleben, das Essverhalten oder zwischenmenschliche Beziehungen (vgl. Foucault 1993, 173ff.; Wiedemann 2016). Aus der zunehmend an Bedeutung gewinnenden Steigerungslogik der Moderne leitet Rosa den Wettbewerb als typischen Interaktionsmodus ab. Wie das Beispiel des Unternehmerischen Selbst zeigt, verändert sich im Zuge der Beschleunigung auch der Status einer Person weg von einem statischen Konzept, als etwas, das man zugewiesen bekommt und danach oftmals ein Leben lang für sich beanspruchen kann, hin zu einer dynamischen Vorstellung der eigenen sozialen Position. Für die Individuen bedeutet das einen zunehmenden Kampf um die Werterhaltung der Person (vgl. Rosa 2006).

Diese Überlegungen machen die immanente Steigerungslogik und expansive Tendenz der westlichen Moderne deutlich und zeichnen darüber hinaus ein Bild der Modernisierung als Rationalisierung, in dem nahezu alle Bereiche des Lebens Effizienz- und Leistungskriterien unterworfen werden können. Die systematische Berechen- und Beherrschbarkeit ist ein zentrales Argument in Rosas Zeitdiagnose und verweist auf das allumfassende Ziel der Profit- und Nutzenmaximierung. Der Einzug dieses rationalen Kalküls in die subjektive und soziale Welt, wie sie Vormbusch et.al beschreiben, wird den Inhalt des nächsten Kapitels bilden (Vormbusch 2019). Nicht nur Rosa, auch andere kritische Soziolog*innen betonen die Unterwerfung der Subjekte unter die Bedingungen des Kapitalismus, ebenso wie unter gesellschaftliche Institutionen, Verhältnisse und Strukturen. Am Ende dieser Entwicklung steht für einige das vollständige Diktat der Markt- und Warenlogik über alle gesellschaftlichen und natürlichen Bereiche. Der Quantifizierung und neuen disruptiven Technologien kommt dabei ein zentraler Stellenwert zu, da sie die umfassende Kommodifizierung und die Übersetzung des Lebens in eine fiktive Ware ${ }^{10}$ erst ermöglichen.

Voraussetzung für diese Ökonomisierung des Lebens sind demnach neue technologische Entwicklungen und die Ausbreitung informationeller Technologien. Die weitreichenden technologischen Durchbrüche der letzten Jahre erleben laut Manuel Castells eine exponentielle Expansion aufgrund der Digitalisierung. Diese ermöglicht es, durch digitale Sprache eine Schnittstelle zwischen technologischen Bereichen zu schaffen, in denen Informationen gespeichert, weiterverarbeitet und weitergeleitet werden (vgl. Castells 2017, 34). Aufgrund der Durchgängigkeit

\footnotetext{
${ }^{10}$ Zum Begriff der fiktiven Ware siehe Polanyi (2017) In „The Great Transformation“ zeigt Plonanyi schon sehr früh, wie sich Prozesse der Kommodifizierung und Auflösung von Institutionen bereits schon früher historisch bedeutsam waren und zeigt darüber hinaus die wichtige Rolle, die technische Neuerungen dabei spielen. Die Kommodifizierung der subjektiven und sozialen Welt gestaltet sich allerdings als historisch neues Phänomen, dass erst durch die Entwicklung von Informationstechnologie in diesem Ausmaß möglich wird.
} 
und der Fähigkeit, Wissen aktiv aus Informationen zu generieren, spricht Castells sogar von der informationstechnologischen Revolution, um auf die durch Technologien der Informationsverarbeitung und Kommunikation hervorgerufenen Veränderungen aufmerksam zu machen. Anders als frühere bahnbrechende Erfindungen kommt der Weiterentwicklung und Verbreitung von Wissen nicht mehr nur eine zentrale Rolle zu, sondern nun wird dieses Wissen verwendet, um neues Wissen zu erzeugen und Geräte zur Informationsverarbeitung und Kommunikation zu schaffen (vgl. ebd., 36). Dabei komme es ,zu einer kumulativen Rückkopplungsspirale zwischen Innovationen und ihrem Einsatz" (ebd.) und schließlich erscheinen Computer, Kommunikationssysteme und Ähnliches als Erweiterung des menschlichen Geistes, da eine enge Verbindung zwischen sozialen Prozessen und den Produktivkräften (respektive der Fähigkeit, Güter und Dienstleistungen zu produzieren und zu verteilen) besteht und so den menschlichen Verstand selbst zu einer unmittelbaren Produktivkraft werden lässt. Ein zweiter entscheidender Faktor bildet dabei die weltweite Verbreitung neuer Technologien in einer relativ kurzen Zeit. Während vorhergehende Transformationen sich meist nur selektiv oder mit deutlicher zeitlicher Verzögerung ausbreiteten, haben sich neue Informationstechnologien innerhalb von weniger als zwei Jahrzehnten weltweit verbreitet und bisher deutet alles auf eine weitere Beschleunigung ihrer Entwicklung und Nutzung hin (vgl. ebd., 38).

Die materielle Grundlage der sogenannten Netzwerkgesellschaft bildet bei Castells mehrere Dimensionen und verdeutlicht wichtige Aspekte, die bei der Betrachtung von Selbstvermessungspraktiken nicht aus dem Blick geraten dürfen. Zum einen wird Information selbst zum entscheidenden Rohstoff der Ökonomie. Informationen bilden eine Antithese zur bisherigen Logik der Verknappung ${ }^{11}$ und werden nicht im herkömmlichen Sinne verbraucht, sondern durch die Verarbeitung von Daten zu Wissen und zu neuen Businessmodellen der informationellen Ökonomie (Castells \& Kößler 2003). Während ein Teil dieser Daten dazu genutzt wird, Produkte und Dienstleistungen zu verbessern, wird der andere Teil zum proprietären Verhaltensüberschuss erklärt (Zuboff 2018). Auf künstliche Intelligenz gestützte Algorithmen berechnen aus diesem Überschuss Vorhersageprodukte, die zukünftige Handlungen und Bedürfnisse entwerfen. Indem menschliche Erfahrung so zu einem immateriellen Rohstoff transformiert wird, entsteht ein Interesse der Wirtschaft daran, dass Menschen möglichst viele Aspekte ihres

\footnotetext{
${ }^{11}$ Andrew Abbott kritisiert an anderer Stelle die oft angenommene Prämisse, das Prinzip der Knappheit als fundamentales Organisationsprinzip der Moderne anzunehmen, wie beispielsweise in der Rational Choice Theorie. Er hält dieser Sichtweise die These entgegen, dass sich in modernen Gesellschaften häufig das gegenteilige Problem, nämlich das des Überflusses beobachten lasse (siehe Abbott 2007; Heintz 2019). Für die Argumentation der Arbeit ist nicht entscheidend, welche grundlegende Dynamik der Moderne letztendlich zugeschrieben wird, entscheidend ist nur die hier aufgeführte Beobachtung, dass sich Daten nicht als Ressourcen im klassischen Sinne - d.h. als knappe Güter - charakterisieren lassen.
} 
Lebens in Daten übersetzen. Umso mehr Daten verfügbar sind, umso besser können zukünftiges Verhalten und damit zukünftige Märkte errechnet werden. „So erpicht wie zahllose Unternehmen darauf sind, auf unser zukünftiges Verhalten zu wetten, haben Überwachungskapitalisten es mittels dieser Operationen zu immensen Wohlstand gebracht“ (ebd., 22). Die Veredlung von Information profitiert dabei von Vernetzung und die neue Konnektivität, sowohl zwischen Individuen als auch zwischen digitalen Systemen, ermöglicht neue kreative Lösungen, die anstatt der Arbeitszeit zur neuen Ware der Arbeiter*innen werden (vgl. Castells 2017).

Diesen Annahmen folgend geht die Arbeit davon aus, dass die Verbreitung informationeller Technologie einen wesentlichen Bestandteil verschiedener Dynamisierungsprozesse bildet, die in diesem Kapitel vorgestellt wurden. Der dynamischen Stabilisierung auf der Mikroebene und der damit verbundenen, an kapitalistische Logiken geknüpften Steigerungs- und Optimierungsimperative kommt dabei ein wichtiger Stellenwert zu.

\subsection{Vergleichen und Bewerten in der Moderne}

Bei der Übersetzung menschlicher Erfahrungen in Daten spielen, wie sich im Folgenden zeigen wird, Vergleichs- und Bewertungspraktiken eine große Rolle. Da in der späteren Analyse der Fokus maßgeblich auf verschiedene Formen von Vergleichen gelegt wird, die sowohl bei der digitalen Selbstvermessung als auch Selbstdarstellung wichtige Funktionen erfüllen, dient dieses Kapital zur begrifflichen und theoretischen Klärung dieser Phänomene. In Bielefeld widmet sich seit einigen Jahren ein Sonderforschungsbereich Vergleichen, allerdings überwiegend aus einer historischen Perspektive. ${ }^{12}$ Spannend für diese Arbeiten sind unter anderem die Arbeiten von Beuscart, Mellet oder Frisch, die sich mit Bewertungspraktiken auf Online-Plattformen wie AirBnB beschäftigt haben (Beuscart \& Mellet 2017; Frisch 2019). Ebenfalls einen Fokus auf digitale Bewertungspraktiken legen Kropf \& Laser in ihrem 2019 erschienenen Sammelband. Darüber hinaus sind die Arbeiten von Bettina Heintz hervorzuheben, die sich in verschiedenen Aufsätzen mit Vergleichen als soziales Phänomen eigener Klasse auseinandersetzt und sich auch ausführlich mit den Vorteilen, die quantitative Vergleiche zu haben scheinen, beschäftigt (Heintz 2007, 2010, 2016, 2019).

Bereits Nietzsche beschrieb seinerzeit das „Zeitalter der Vergleichung“ (Nietzsche 2005) und vor dem bereits behandelten Hintergrund scheint das durchaus plausibel, denn die skizzierte Auflösung traditioneller Ordnungsstrukturen fordert neue Mittel sozialer Positionierung ein.

${ }^{12}$ Zur Website des Sonderforschungsbereichs: https://www.uni-bielefeld.de/sfb1288/ (zuletzt aufgerufen am 20.08.2020), siehe auch (Arlinghaus, Erhart \& Gumpert 2020; Becker et al. 2015; Davy et al. 2019). 
Auch im Kontext der Globalisierung und Digitalisierung hängt das internationale Agieren von Märkten davon ab, dass die verschiedenen Akteure untereinander vergleichbar sind, um sich wechselseitig beobachten und anpassen zu können (vgl. Heintz 2016; Vormbusch 2004). Trotz ihrer alltäglichen Relevanz wird Vergleichen allerdings in der bisherigen Theoriebildung meist nur eine untergeordnete Rolle zugewiesen, weshalb es zunächst notwendig erscheint zu klären, wodurch sich Vergleiche auszeichnen.

\section{Was und wofür Vergleiche?}

Grundlegend benötigen Vergleiche zwei Elemente: eine Gleichheitsunterstellung und eine Differenzbeobachtung. Vergleiche haben die Funktion, Sachverhalte anhand einer dritten Größe auf ihre Unterschiede oder Gemeinsamkeiten hin zu beobachten und in Beziehung zueinander zu setzen. Um diesbezüglich eine Aussage treffen zu können, braucht es über ein Vergleichskriterium hinaus noch ein Verfahren, durch das sich die potenziellen Differenzen ermitteln lassen (vgl. Heintz 2016, 307). Dadurch, dass der Vergleich einen gemeinsamen Sinnzusammenhang zwischen zwei Entitäten erzeugt, dient er simultan als Instrument und Technik zur Unterscheidung und Relationierung. Die Kommensurabilität ist dabei keine Eigenschaft, die in den Dingen selbst verankert ist, sondern entsteht vor dem Hintergrund sozialer und kultureller Praktiken. Besonders wirksam können Vergleiche dadurch werden, dass sie öffentlich kommuniziert werden und dadurch für Dritte sichtbar sind. Die Ansprüche an Wachstum und Perfektionierung führen zusätzlich eine zeitliche Perspektive ein, die einen Selbstvergleich möglich macht. Die Verbreitung von Selbstvermessungstechnologien zur Sichtbarmachung des persönlichen (Leistungs-)Fortschritts sind dabei nur ein Beispiel für die Aktualität und Bedeutung von Vergleichen.

Die bereits behandelte Auflösung traditioneller Bindungen und die daraus erwachsende Gleichheitssemantik begründet die Modernität des Vergleichs. Durch die Auflösung (zumindest zu Teilen) vormoderner Denkstrukturen wird „,das Wesen der Dinge“ Gegenstand aktiver Aushandlungsprozesse. Dabei führe der „Sog der Relativierung“ zu einer Reflexionsspirale der Vergleiche (ebd., 311). Seit dem Beginn des 18. Jahrhunderts lasse sich so eine Ausdehnung des Vergleichs, sei es auf räumlicher, zeitlicher, sozialer oder sachlicher Ebene, beobachten. Eng damit verknüpft ist eine neue Unsicherheit über den Wert und Uwe Vormbusch konstatiert in diesem Zusammenhang eine Krise der objektiven Bestimmbarkeit. Wenn der Wert bestimmter Objekte hinterfragbar wird, konkurrieren unterschiedliche Bewertungspraktiken miteinander und müssen zum Gegenstand reflexiver Aushandlungen werden. „Bewertungen treten also nicht nur am Aktienmarkt im Sinne der Bewältigung kognitiver Unsicherheiten auf. Stattdessen 
reflektieren sie die Vielfalt der Lebenspraktiken sowie moralische Unsicherheit moderner Gesellschaften - und sind das Medium, in dem diese Unsicherheiten verhandelt werden." (Vormbusch 2019, 27) Damit wird die Alltagspraxis des Vergleichens und Bewertens und die Rechtfertigung zugrundeliegender Maßstäbe zum allgegenwärtigen Handlungsproblem für die Subjekte und alles und jeder kann auf seinen Wert hin befragt werden, da immer auch Alternativen denkbar sind (vgl. ebd.). Die Attraktivität der Vergleiche liegt also in ihrer Fähigkeit, Unterschiede sichtbar zu machen und dadurch dem Generalverdacht der Kontingenz ${ }^{13}$ zu entkommen. Vergleiche dienen somit als niederschwellige Ordnungsgeneratoren, die nur dann auf Probleme stoßen, wenn Dinge eine gewisse Ambiguität aufweisen und sich einer eindeutigen Zuordnung entziehen (vgl. Heintz 2016, 314).

Zusammenfassend kann gesagt werden: Vergleiche sind ein konstitutives Moment sozialer Ordnung - wie sonst könnten sich Menschen in ihrer sozialen Wirklichkeit orientieren, zurechtfinden und in Bezug zu anderen Menschen betrachten?

\section{Der Reiz quantitativer Vergleiche}

Eine Voraussetzung, um sich soziologisch mit Vergleichen zu befassen, ist die Betrachtung desselben als kommunikatives Phänomen und nicht als kognitiver Akt. Daraus kann dann abgeleitet werden, dass ein Vergleich erst sozial anschlussfähig ist, wenn er kommuniziert wird Möchte man in einem nächsten Schritt quantitative Vergleiche von anderen abgrenzen, muss darüber hinaus davon ausgegangen werden, dass einzelne Kommunikationsformen eine Eigenlogik besitzen, dass es also einen signifikanten Unterschied macht, wie etwas kommuniziert wird. Ausgehend von diesen Grundannahmen kann dann die These formuliert werden, dass quantitative Vergleiche (in einer technokratisch-wissenschaftsbasierten Gesellschaftsform) eher Anschluss finden als sprachlich formulierte (vgl. Heintz 2010, 163). Quantifizierung erhört die Überzeugungskraft von Aussagen, weshalb für ihre Falsifizierung oder Relativierung meist alternative Zahlen erwartet werden (vgl. Zillien, Fröhlich \& Dötsch 2015, 81). Genau lässt sich die These der Anschlussfähigkeit von numerischen Aussagen anhand von vier Dimensionen begründen. Die erste Dimension beinhaltet Erzeugungsregeln, also Regeln und zulässige Kombinationen verschiedener Zeichen, die nicht überall gleich sind, sondern sich darin unterscheiden, wie explizit, selektiv und normiert sie sind. Während also zum Beispiel in der Sprache

\footnotetext{
${ }^{13}$ Die Annahme, dass in der Moderne prinzipiell alles hinterfragbar wird und somit hier von einem Generalverdacht der Kontingenz gesprochen werden kann, begründet sich im Übergang von einer stratifikatorischen hin zu einer funktionellen Differenzierung und der damit verbundenen Infragestellung festgefügter Ordnungsstrukturen. „Die Ordnung der Dinge“ (Foucault 1966) lässt sich nicht länger in ihnen selbst verorten, sondern ist Gegenstand fortlaufender Aushandlungs-, Stabilisations- und Naturalisierungsprozesse (vgl. Heintz 2007, 2016).
} 
bestimmte grammatikalische Regeln relativ explizit vorgeben, wie man Wörter aneinanderreihen darf, sind in der modernen Kunst weitaus mehr Kombinationsmöglichkeiten zulässig und ungewöhnliche Zusammenstellungen meistens sogar erwünscht. Rechenverfahren, mathematische Operationen und Ziffern sind sehr stark und eindeutig festgelegt, was zwar ihre Variation einschränkt, sie dabei aber auch sehr selbstexplikativ werden lässt. Sie brauchen wenig Kommunikation über sich selbst, da die Erzeugungsregeln die Kontingenz in weiten Bereichen blockiert und somit auch (und das ist das für diese Arbeit entscheidende) Zustimmung wahrscheinlicher macht. Plakativ formuliert ist also eine Darstellung der eigenen Fitness in Form von Zahlen und Kurven anschlussfähiger als die Darstellung desselben als expressives Kunstwerk oder als Musikstück. Eine Falsifizierung von Zahlen fällt meist sehr schwer, da das Verfahren ihrer Herstellung und ihre Ressourcen entweder nicht bekannt sind oder nicht widerlegt werden können (vgl. Heintz 2010, 172). Daneben steht nicht zur Diskussion, was eine Zahl ist, entscheidend ist nur, nach welchen Regeln mit ihr umgegangen wird. Mathematische Operationen sind also in hohem Grad selbstreferenziell, da sich Zahlen meist nur noch auf andere Zahlen berufen, ohne einen „realen“ Bezugspunkt zu haben. „Die geringe Indexikalität quantitativer Darstellungen, ihre ,disembeddedness', erleichtert ihre Anschlussfähigkeit in kulturell heterogenen Kontexten“ (ebd., 173), was die Verständigung oftmals erleichtern kann. Im Kontrast dazu sind verbale Äußerungen stark an den soziokulturellen Hintergrund gebunden und können sich je nach Region und Schicht stark voneinander unterscheiden. Schlussendlich werden Kommunikationsmedien in ihrer externalen und internalen Zurechnung unterschieden. Entweder wird mit der Ausdrucksweise suggeriert, dass es sich um einen „objektiven“ Sachverhalt handelt, der erlebt wird, oder, dass eine Zurechnung auf subjektive Präferenzen, Interessen und Motive naheliegend ist. Verbale oder nonverbale Äußerungen sind durch ihren persönlichen Charakter hinterfragbar und potenzielle Zweifel an der Aussage können zwar reduziert werden, sie präsentieren sich aber nie als logisch zwingend.

Zusammenfassend wird deutlich, wie der geringe Interpretationsspielraum und die universale Deutbarkeit Zahlen einen „Vorteil““ gegenüber narrativen oder nonverbalen Äußerungen verschaffen können (vgl. ebd., 174). Darüber hinaus produzieren Quantifizierungsprozesse Sichtbarkeit und führen oftmals erst zur sozialen Konstruktion von Realität. Selbstvermessungspraktiken machen Dinge auf eine objektivierende Weise sichtbar, die ähnlich zum Accounting in der Wirtschaft einen Aufschwung erfährt (vgl. Vormbusch 2004, 36). ${ }^{14}$

\footnotetext{
${ }^{14}$ Die Accountingforschung, die vor allem im angelsächsischen Raum anzusiedeln ist, beschäftigt sich unter anderem mit der Selbstbeschreibung kapitalistischer Gesellschaften in Form von kalkulativen Konstrukten. Die Zahlen spielen dabei eine zentrale Rolle und konstruieren sowohl auf Organisations- als auch Subjektebene soziale
} 


\section{Soziokalkulation}

Die Verbreitung des gesellschaftlichen Zahlengebrauchs und die allgemeine Unsicherheit über den Wert führen zu einer neuen Institutionalisierung von Bewertungen. Die neu entstehenden systematischen Formen der Bewertung, wie beispielsweise Hochschulrankings, PISA-Studien oder Restaurantbewertungsplattformen, wirken dabei auf die Subjekte und ihre Lebenswelt zurück und entfalten subjektivierende Machtwirkung, indem vielfältige Bewertungspraktiken zunehmend in den Alltag integriert werden (Frisch 2019; vgl. Vormbusch 2019, 29). Die Quantifizierung dehnt sich aus und hält auch Einzug in die subjektive und soziale Welt, wenn Freunde auf sozialen Medien in quantifizierten Listen dargestellt werden und der Körper zunehmend in Zahlen übersetzt wird. In diesem Licht erscheinen evozierende Accountingprozesse nicht als neutrale Sach-, sondern im Sinne Foucaults als Regierungstechnologie, in deren Mittelpunkt die Führung des Menschen steht. Durch innovative Praktiken wird bisher Inkommensurables für Bewertungen zugänglich gemacht und zum legitimen Gegenstand von Kalkulationen (vgl. ebd., 33). Der Begriff der Soziokalkulation verdeutlicht die Ausdehnung ökonomischer Kalkulationspraktiken und beschreibt eine soziale Praxis, deren gezieltes Erkenntnisobjekt Phänomene der sozialen und subjektiven Welt bilden. Empirisch ist daran die Annahme eines ökonomischen Strukturwandels geknüpft, der von einem Bedeutungsgewinn immaterieller Ressourcen und einer Zunahme normativer Unsicherheit ausgeht. Dabei geht Vormbusch davon aus, dass die Wahrnehmung einer wachsenden Unsicherheit zwar durchaus einen Bezugspunkt für soziale Suchprozesse darstellt, diese aber nicht zwangsläufig erfolgreich sein müssen (vgl. ebd., $34)$.

Selbstvermessungspraktiken weiten den Bewertungshorizont aus, indem nicht mehr nur die immaterielle Arbeit in formalen Organisationen Zielscheibe derselben ist, sondern jetzt auch die alltägliche Lebensführung und die verborgenen Qualitäten der Subjekte und ihrer Körper relevant werden. Die Selbstvermessung geht nach Vormbusch auf eine für die Moderne konstitutive Kultur des Messens und allgegenwärtigen Vergleichens zurück und steht auch vor dem Hintergrund des „neuen Geistes des Kapitalismus“ (Boltanski, Chiapello \& Schultheis 2006) sowie dem Imperativ der Selbstverwirklichung, Autonomiesteigerung und Vernetzung. Die Auseinandersetzung mit sich selbst ist damit Teil des kulturellen Kapitals und der „Erfolgskultur“ der Gegenwart (Vormbusch 2019, 36f.). Wie im folgenden Kapitel deutlich werden wird, wird

Wirklichkeit. Diese Forschungsrichtung legt dabei eher einen Fokus auf die Mesoebene und betrachtet beispielsweise Phänomene auf dem Finanzmarkt oder in Verwaltungen. Allerdings sind die Ergebnisse aus Überlegungen zur Quantifizierung in der Wirtschaft auch für diese Arbeit interessant, wieso an dieser Stelle darauf Bezug genommen wird. Näheres über die Accountingforschung in Vormbusch 2004. 
bei Untersuchungen zu Self-Tracking häufig der Fokus auf die Quantifizierung gelegt und die Bedeutung der Vergleiche treten in den Hintergrund. Teil der Arbeit ist es, diese vorgenommene Gewichtung kritisch zu beleuchten und durch die Untersuchungen qualitativer Selbstdarstellung auf Instagram einer Prüfung zu unterziehen. 


\section{Digitale Selbstvermessung}

Die bestehende Literatur zur digitalen Selbstvermessung bzw. zum Lifelogging beschränkt sich bisher auf quantitative Formen der Selbstvermessung und -thematisierung. Oft stehen dabei Risiken und Gefahren im Mittelpunkt, die sich aus der Übersetzung des Lebens in quantitative Daten ergeben. Beispielhaft für eine solche kritische Betrachtung mit oft dystopischem Ausblick sind Aufsätze, die in den emergierenden Selbstvermessungspraktiken eine Verabsolutierung neoliberaler Narrative sehen, denen es durch Self-Tracking-Devices gelingt, nunmehr alle Bereiche des menschlichen Lebens unter die Logiken von Markt, Wettbewerb und Konkurrenz zu unterwerfen (vgl. Selke 2016a). Da bisher eher wenige Studien einen empirischen Fokus legen und viele der Arbeiten zu digitaler Selbstvermessung einen theoretisierenden Schwerpunkt haben, werden im Folgenden anstelle einer klassischen Aufarbeitung des Forschungsstandes theoretische Erkenntnisse zu Self-Tracking zusammengefasst und dann in einem zweiten Abschnitt auf das Konzept von Stefan Selke zugespitzt werden.

\subsection{Bedeutungsproduktion durch digitale Selbstvermessung}

Selbstvermessungstechnologien eröffnen neue Möglichkeiten der Selbstoptimierung und Effizienzsteigerung in nahezu allen Bereichen des Lebens, sei es Sport, den eigenen Gefühlen, dem Schlaf oder dem Sozialleben. Die „Quantifed Self“-Bewegung stellt aus dieser Perspektive eine Reaktion auf fortlaufend steigende Leistungsanforderungen und ständiges Wachstum dar, wobei die technisch unterstütze Selbstvermessung dabei helfen soll, die Grenzen des eigenen Körpers auszuweiten, um weiterhin den Wachstumsimperativen der Moderne standzuhalten. Ganz im Sinne neoliberaler Denkweisen funktioniert die Selbstvermessung auf der individuellen Ebene und klammert gesellschaftliche Problemlagen aus (vgl. Stark 2016, 288). Die Gesamtfunktion des „Quantified Selfs“ bestehe darin, dass „der Mensch [...] in ein Ordnungs- und Leistungssystem von Überwachungsanwendungen, technischen Geräten und datengestützter Ergebnisauswertung eingebunden [wird]“ (ebd., 299). Die Tatsache, dass die Menschen dabei keiner Außenlenkung ausgesetzt sind, sondern von innen heraus den Leistungsimperativen folgen ist, laut Stark, nur ein weiteres Indiz für die Durchsetzungsfähigkeit der neoliberalen Leistungsgesellschaft (vgl. ebd., 293). 


\begin{abstract}
„Die ,bonbonbunte“ Optik von Fitbit.com kollidiert bei genauer Betrachtung mit der technokratischen Unerbittlichkeit, eines selbstauferlegten Zwangs zur Leistungserbringung. Es spiegelt sich an dieser Stelle auch der Trend zur sogenannten Gamification wider. Es handelt sich dabei um die Übernahme spielerischer Elemente in alle möglichen Lebensbereiche. Symptome für diese Entwicklung sind etwa exzessive Ranglistenaufstellungen oder virtuelle Medaillen und 'Abzeichen', also virtuelle Belohnungen, die allesamt leistungssteigernd und zugleich verharmlosend wirken sollen.“ (ebd., 297)
\end{abstract}

Die Deutung Selbstvermessungstechnologien als Mittel zur Selbstoptimierung bildet einen weitestgehenden Konsens, wenngleich ähnliche Arbeiten sich etwas differenzierter mit der Bedeutungsproduktion durch Self-Tracking beschäftigen. So geht auch das Projekt „Das vermessene Leben“" von einer auf quantitative Steigerung ausgerichteten Optimierungslogik aus, die sich unter anderem bei Selbstvermesser*innen finden lässt. In den Blick gerät das Subjekt dabei als zunehmend vermess- und kalkulierbares, das sich neuen Bewertungs- und Vergleichsordnungen gegenübersieht (Vormbusch \& Kappler 2016). Neben der Quantifizierung und Objektivierung des Selbst durch Selbstvermessung nehmen Zillien et.al. einen Prozess reflexiver Selbstverwissenschaftlichung wahr und beobachten eine zunehmende Expertisierung beispielsweise innerhalb des Diät-Trackings (Heyen 2019; Zillien, Kofahl \& Fröhlich 2016).

Mit Bedeutungsproduktion durch digitale Selbstvermessungspraktiken beschäftigt sich Stefanie Duttweiler. Für sie besteht die Besonderheit quantitativer Selbstvermessung in der Komplexitätsreduktion des Lebens. Im Anschluss an Bettina Heintz betont sie die Fähigkeit der Zahlen, Objektivität zu signalisieren und dadurch zu einer Kontingenzreduktion beizutragen. Allerdings verbleibt sie, ähnlich wie Heintz, nicht bei dieser Erkenntnis, sondern zeigt, dass dieser Objektivierungsprozess nicht von der Bedeutungsgebung der selbsterhobenen Zahlen und Daten zu trennen ist. Daten und Zahlen stehen niemals für sich und müssen subjektiv bedeutend gemacht werden. Praktiken der Selbstvermessung sind dadurch immer Praktiken der Bedeutungsgebung, denn schon beim Vermessungsprozess selbst findet durch die Selektion der erhobenen Parameter oder der Messzeitpunkte eine gezielte Auswahl statt. Anhand qualitativer Interviews mit Sportstudierenden stellt sie so die These auf, dass sich Self-Tracking als wechselseitige Bedeutungsproduktion von Daten und Subjekten gestaltet (Duttweiler 2018, 252-54). Bereits die Produktion der Daten innerhalb des Self-Tracking ist voraussetzungsvoll und setzt einen gewissen „Willen zum Wissen“ voraus (Foucault 1991). Es müssen gewisse Messparameter, ein geeignetes Gerät und passende Situationen, die sich zu einer Erhebung eignen, aus- 
gesucht werden. Unter den von ihr befragten Personen zeigt sich ein langer Prozess der Etablierung von Routinen sowie Auswahl und Anpassungsprozesse, aber auch Entscheidungen darüber, mit wem man die erhobenen Daten teilt und wie weit man die Messpraktiken in den Alltag integriert (vgl. Duttweiler 2018, 255). Je nach Motivation unterscheidet sich die Art und Weise, wie vermessen wird. Als Motive arbeitet Duttweiler den Wunsch nach individuellen und sozialen Vergleichen, den Wunsch nach einer Leistungssteigerung und die Effizienzsteigerung des Trainings heraus und betont, wie sehr für die meisten Befragten das Messen mit der Praxis verknüpft ist. Zahlen fungieren dadurch zum einen als Medium der alltäglichen Selbstthematisierung, zum anderen werden die Zahlen und Kalkulationen zur Selbsterforschung genutzt (vgl. Noji \& Vormbusch 2018).

Darüber hinaus schaffen es Zahlen, Bewegungsaktivitäten in sportliche Leistungen zu verwandeln, indem sie ihnen Wert verleihen (vgl. Duttweiler et al. 2016, 222). Umgekehrt wird auch nur vermessen, was als sportliche Leistung definiert wird: So wird die Fahrradstrecke zur Universität nicht als Sport wahrgenommen, während die Laufstrecke, mit dem Ziel eine Leistung zu erbringen, eher als Sport bezeichnet und damit auch aufzeichnungsrelevant wird (vgl. Duttweiler 2018, 257). Fitnesspraktiken werden somit zu Erfolgspraktiken der Körper- und Selbstermächtigung. Dadurch ist es wichtig, dass sich „Fit-Sein“ zeigt, um daraus soziale Anerkennung und Distinktion generieren zu können (vgl. Duttweiler et al. 2016, 222ff.). Die Sichtbarmachung sportlicher Leistungen und der dadurch mögliche Vergleich mit anderen spielt also eine zentrale Rolle bei der Selbstvermessung. Neben Leistung und Vergleich sind auch normalisierende Diskurse wichtig, wenn für einen der Befragten der Körper wichtiger Bestandteil seiner beruflichen Identität und zur Visitenkarte wird (vgl. Duttweiler 2018, 259).

Auch der Glaube an sie ist den Zahlen nicht immanent, sondern ist Bestandteil von Aushandlungsprozessen und subjektiver Bedeutungsgebung.

„Wie die Wissenschafts- und Technikforschung an vielen Beispielen herausgearbeitet hat, ist die unterstellte Objektivität und die mit ihr verbundene Vertrauenswürdigkeit von Zahlen und wissenschaftlichen Daten Effekt einer komplexen, historisch gewachsenen, sozialen Praxis der Datenerhebung und -interpretation.“ (ebd., 260)

Das Vertrauen in die Zahl ist demnach auch nicht selbstverständlich und spiegelt sich beispielsweise in zahlreichen Foren und Bewertungskommentaren zu Fitnesstrackern. Den gewonnenen Daten wird nicht, wie manche Arbeiten vermuten lassen würden, blind vertraut, vielmehr wird die vermeintliche Objektivität in den Nutzungspraktiken problematisiert und teilweise auch nur 
bedingt angestrebt. Stefanie Duttweiler kristallisiert in diesem Zusammenhang zwei Umgangsweisen heraus: Zum einen wird versucht, durch Korrektive und Überprüfung der Daten sich der Objektivität anzunähern, zum anderen wird hingenommen, dass es sich bei den Zahlen um Näherungswerte handelt, die kein exaktes Abbild der Realität liefern können. Je nach Situation kann darüber hinaus die Zahl unterschiedlich bewertet oder ihre Bedeutung relativiert werden (vgl. ebd., 263).

Im Gegensatz zur Datenerhebung wird die Interpretation der Fitnessdaten weniger problematisiert und es fällt den Befragten leicht, den erhobenen Daten eine Bedeutung zuzuschreiben. Verschiedene Kennzeilen (wie beispielsweise die NikeFuels) helfen dabei, die Vergleichbarkeit zu erhöhen. ${ }^{15}$ Die Listen, Kurven und Diagramme der Fitness-Apps legen zusätzlich eine bestimmte Interpretation der Daten nahe, auch Symboliken wie Ampeln, Pfeile oder Thermometer rahmen die Interpretationsmöglichkeiten der Daten. Die explizit normative Darstellung der Messdaten ordnet die Menschen und ihre Leistungen in Bewertungssysteme ein, wodurch die dargestellten Daten immer auch vermittelt durch gesellschaftliche Normen und Werte angezeigt werden (vgl. ebd., 265). Werden die Daten, wie bei den NikeFuels, zu übergeordneten Kennzahlen verarbeitet, entstehen Black Boxes, die die Komplexität und Kontingenz ihrer Herstellung ausblenden. Oftmals werden diese Kennzahlen ergänzend in bildliche Symbole übersetzt und gehen damit über die rein quantitative Vermessung hinaus. Das zeigt, dass die vermessene Leistung weder rein automatisch noch rein zahlenbasiert funktioniert, sondern meist ergänzungsbedürftig ist. Vor allem, wenn die Vermessung von Gefühlen oder Stimmungen relevant wird, sind die technischen Geräte auf die subjektive und manuelle Eingabe der Nutzenden angewiesen.

Die in der Praxis kaum hinterfragte Evidenz der Zahlen entsteht demnach zum einen durch den Verweis auf (vermeintliche) Wissenschaftlichkeit und zum anderen durch die vermeintlich unmittelbare Repräsentation der Wirklichkeit durch Bilder und Grafiken. „Durch den Rückgriff auf bekannte Steuerungs- und Bewertungssysteme werden damit nicht zuletzt auch die normativen Implikationen unmittelbar evident und kaum hinterfragbar“" (ebd., 266).

Darüber hinaus lässt sich eine unmittelbare emotionale Bewertung innerhalb des Rahmens der eingeschriebenen normativen Bewertung beobachten. Dass den Daten nie neutral entgegenge-

\footnotetext{
${ }^{15}$ Espenland und Stevens arbeiten in diesem Zusammenhang heraus, dass Vergleichbarkeit eine spezifische Art der Beziehung zwischen Objekten erzeugt und dazu neigt, Differenzen in Quantitäten zu übersetzen. „In doing so, it unites objects by encompassing them under a shared cognitive system“ (Espeland \& Stevens 2008).
} 
sehen wird, zeigt die Übersetzung der Zahlen in Narrationen, die eng verknüpft sind mit Diskursen um Schönheitsideale oder einem guten Leben. ${ }^{16}$ Spannend, auch in Bezug auf den empirischen Teil der Arbeit, ist die Bedeutsamkeit, die Daten alltäglichen Handlungen verleihen können. Durch das Vermessen gewinnen Praktiken im Alltag eine neue Bedeutung, sie ,zählen“ gewissermaßen. Das Erheben von Daten erzeugt nicht nur deskriptive Fakten, sondern verändert performativ den Wert derselben (vgl. ebd., 269). Neben dem „Update“, das die Handlungen durch ihre Vermessung erhalten, können die Daten auch Antworten auf Identitätsfragen liefern. Dadurch können sie dabei helfen, die eigene soziale Position zu ermitteln und bilden somit auch eine Ressource für sozialer Anerkennung. Durch Self-Tracking wird der Körper einmal mehr zum Instrument und Objekt der Selbstdisziplinierung. Die Sichtbarmachung der Leistung und ihr Festhalten für spätere Zeitpunkte wird in diesem Kontext attraktiv und bedeutsam, die zeitliche Dimension macht darüber hinaus auch Fortschritte und Wandel sicht- und greifbar. Daten geben also einen neuen Rahmen für Praktiken und Handlungsweisen, vermeintlich objektive Antworten auf Identitätsfragen und dokumentieren die Sorge um sich (vgl. ebd., 272).

Bei der Selbstvermessung spielen leib- und subjektgebundene Kompetenzen des Unternehmerischen Selbst eine große Rolle, nicht aus dem Blick geraten dürfen dabei aber auch das politische Interesse der Erzeugung einer immensen Menge an personenbezogenen Daten, die teilweise ungehindert im Netzt geteilt werden.

\begin{abstract}
„Bevor aber Selbstvermesser Aspekte ihres Selbst und ihres Tun einen Wert zuweisen können, müssen Taxonomien etabliert werden und eine gewisse Dauerhaftigkeit erlangen. Bewertungsordnungen beschränken sich nicht nur auf kognitive Operationen, sondern sind kollektiv geteilte Vorstellungen des Wünschenswerten und Guten und damit auch nicht vollständig von moralischen Gesichtspunkten zu trennen.“(Vormbusch 2019, 38)
\end{abstract}

\footnotetext{
${ }^{16}$ Auch andere Studien zeigen, dass zum Beispiel Daten über das eigene Körpergewicht niemals neutral begegnet wird, sondern sie immer mit einer emotionalen Wertung versehen und zwischen „gut" und ,schlecht" verortet werden. Mindestens genauso bedeutend wie der eigene Fortschritt ist für die Befragten der Vergleich mit anderen, da er die eigene Position anzeigt (vgl. Duttweiler 2018, 268). Das verdeutlicht auch folgendes Zitat: „Und auch das eigene Körpergewicht oder der Körperfettanteil sind keine neutralen Daten, sondern sozial bedeutsam, enthalten sie doch immer auch eine Bewertung über Normalität und Abweichung und somit über die Positionierung in der Gesellschaft (vgl. Barlösius 2014; Zitiert nach Duttweiler). In dem Moment, in dem man auf der Waage steht, wird diese Position unerbittlich angezeigt“" (Duttweiler 2018, 269).
} 


\subsection{Lifelogging}

Da Stefan Selkes Konzept des Lifelogging zentral für die vorliegende Arbeit ist, wird sein Beitrag zur theoretischen Beschreibung von digitaler Selbstvermessung in diesem Kapitel gesondert vorgestellt. Selke legt ebenfalls einen Fokus auf Leistungs- und Optimierungsaspekte und behandelt in seiner Analyse sowohl Potenziale als auch Pathologien. Lifelogging wird bei ihm definiert als „die Summe aller Versuche, menschliches Leben in Echtzeit zu erfassen, in dem heterogene Verhaltens- und Datenspuren digital aufgezeichnet, in einem Speicher abgelegt und zum späteren Wiederaufruf und zur Visualisierung vorrätig gehalten werden“" (Selke 2019, 324). Für ihn zentral ist darüber hinaus die Suche nach Ordnung und Struktur, aber auch die Vorstellung des Menschen als strukturell fehlerhaft. Während Selbstthematisierung an sich kein neues Phänomen ist, identifiziert Selke als neues Moment der Selbstvermessung die Dynamisierung und Vernetzung sowie die Ökonomisierung der Nutzung. Damit schließt er an die bereits vorgestellte Theorie der Beschleunigung an. Ausgangspunkt seiner Überlegungen bilden neben Rosas Theorie die Verflüssigung der Moderne wie auch verschiedene Erschöpfungsdiagnosen. ${ }^{17}$

Spannend an Stefan Selkes Herangehensweise ist der Einbezug der Technik in seine Überlegungen. So würden Lifelogging-Technologien als Teil der digitalen Transformation mit bestehenden Wertevorstellungen brechen, etwa durch die Auflösung der Privatsphäre oder dem stärkeren Fokus auf die Eigenverantwortung der Menschen (vgl. ebd., 325). Dabei beschreibt er Lifelogging-Technologien als emerging technologies, die sich ubiquitär verbreiten und die darin verarbeiteten digitale Speichermedien zunehmend leistungsfähiger, dabei aber auch preisgünstiger werden. Die körpernahen Aufzeichnungsgeräte dringen in Folge dessen zunehmend in den Körper ein. Die Funktionalität von Lifelogging-Technologien kann entlang einer Prozesskette verstanden werden und macht deren Heterogenität deutlich. Von der Datenerfassung über die Speicherung und Datenanreichung (content processing) sowie die Möglichkeit des späteren Wiederabrufs der Daten verläuft die Kette bis hin zu Visualisierung (beispielsweise auf dem Smartphone). ${ }^{18}$ Wirksam wird die Technologie laut Selke in einem sozialen und einem kognitiven Rahmen, was zeigt, dass er mit seinem Konzept versucht, eine kulturalistische mit einer technizistischen Sichtweise zu vereinen. So sind neben der Annahme, dass die Geräte und

\footnotetext{
${ }^{17}$ Zum theoretischen Hintergrund Selkes siehe unter anderem: Bauman \& Jakubzik 2007; Neckel 2005; Rosa 2016a.

${ }^{18}$ Stefan Selke skizziert an dieser Stelle verschiedene Unterscheidungen, die anhand dieser Prozesskette getroffen werden können: Der Aufwand der Datenerfassung und die Datenmenge unterscheide Lifelogging-Technologien ebenso wie der Modus der Relevanzsetzung (aktiv/passiv, deterministisch/probabilistisch) oder auch der Grad der Tauschbeziehung (individuell/vernetzt) (vgl. Selke 2019, 326).
} 
die selbst erzeugten Daten eine objektive Messbarkeit ermöglichen, die Einbettung der Technologie in Konventionen und typische Gebrauchsweisen grundlegender Bestandteil des Lifeloggings (vgl. ebd., 325f.). Durch die Verbreitung verschiedenster Fitness-Apps und Wearables hat Lifelogging Einzug in den Alltag vieler Menschen gehalten. Nichtsdestotrotz steht die Verwendung von Selbstvermessungstechnologien und -anwendungen noch weit hinter Social-Media-Anwendungen zurück. ${ }^{19}$

Der Technik wird in dieser Arbeit auch immer eine soziale Dimension zugesprochen, sie ist selbst weder gut noch schlecht, aber auch nicht neutral. Anschließend an Castells könnte man ergänzen: Die Technik bildet eine Macht, die das Leben und den Verstand durchzieht. „Aber ihre tatsächlichen Entwicklungen im Bereich bewussten sozialen Handelns und die komplexe Matrix der Interaktion zwischen den technologischen Mächten, die unsere Spezies entfesselt hat, und der Spezies selber sind Gegenstände der Forschung und nicht des Schicksals“"(Castells 2017, 87f.). In der Praxis ergeben sich demnach für Stefan Selke verschiedene Potenziale, aber auch Pathologien der Technik, wobei die negativen Aspekte der Technik für ihn deutlich überwiegen.

Bereits angeklungen ist die Möglichkeit, durch Self-Tracking den eigenen Körper zu ver-objektivieren und durch den vermeintlich neutralen Blick auf den Körper die eigene Leistung zu verbessern. Objektive Daten können dabei helfen, den Körper (und den Geist) gezielt zu formen und zu optimieren. Die Optimierung bildet ein zentrales Versprechen des Lifelogging und erklärt das Leben zu einem permanenten Optimierungsprojekt, das einhergeht mit einer gesteigerten Selbsterkenntnis, -beobachtung und -veränderung. Oftmals - vor allem im Gesundheitsbereich - wird die Möglichkeit eines demokratischen Wissenstransfers durch selbsterzeugte Daten betont. ${ }^{20}$ Lifelogging führe zu neuen Aushandlungen der Produktion und Konsumption digitaler Daten und Verfechter sehen die Chance zu einer größeren Teilhabe und Mitbestimmungsrechten.

Der gesteigerten Teilhabe und Demokratisierung, die durch Lifelogging entstehen kann, steht allerdings eine konkrete Ökonomie und eine Konkurrenzmentalität gegenüber. Wenn alle Bereiche des Lebens mess- und damit kalkulierbar werden, wird der Mensch zu einer „Lebendbewerbung“. Lifelogging bildet ein Medium zur alltäglichen Selbstthematisierung und -erforschung. Mithilfe verschiedener Messwerte wird versucht, das vermeintlich authentische Ich zur

\footnotetext{
${ }^{19} \mathrm{https} / / / \mathrm{de}$. statista.com/prognosen/793227/umfrage-in-deutschland-zum-besitz-von-wearables (zuletzt aufgerufen am 04.09.2020).

${ }^{20} \mathrm{Zu}$ personalisierter Medizin und zur kritischen Betrachtung des Lifelogging im Gesundheitssektor siehe beispielsweise (Heyen, Dickel \& Brüninghaus 2019; Prainsack 2020; Selke 2016b).
} 
Darstellung zu bringen und durch die so generierte Sichtbarkeit an sich selbst zu arbeiten, oder sich im Vergleich zu anderen zu messen. Durch die Selbstthematisierung in Form von Zahlen und Kalkulationen wird Lifelogging zu einer Institution der dynamischen Stabilisierung (vgl. Noji \& Vormbusch 2018, 28) und macht die Menschen zu Objekten, die nach ökonomischen Kriterien behandelt werden können. Durch die fortlaufende Einschreibung verschiedener Aspekte in den Körper, verorten sich die Subjekte immer wieder selbst, wodurch Wettbewerbsund Konkurrenzlogiken sich ausdehnen. Durch die mittels Zahlen erzeugte Objektivitätsfiktion und scheinbar universelle Vergleichbarkeit erhält die Gegenwart eine gewisse Solidität und die so kalkulativ gerahmten Subjekte richten sich zunehmend entlang von Steigerungs- und Optimierungsimperativen aus.

Neben der Transformation der Menschen in kommensurable Güter (Vormbusch 2012, 218) führt die Übersetzung des Lebens in (quantitative) Daten zu einer Verschiebung der Sichtweise auf den Menschen und seinen Körper. Laut Stefan Selke wird der soziale Blick auf den Menschen zunehmend rationalisiert und diese quantitative Sichtweise ist anfällig für Kategorienfehler und Komplexitätsverluste. Da sich in der Logik des Lifelogging nur das verbessern lässt, was auch messbar ist, entwickelt sich ein alternativloser Glaube an Scores und Rankings (vgl. Selke 2019, 329). Die Kombination aus (öffentlichem) Vergleich und Quantifizierungsprozessen erwirkt zum einen eine Kontingenzreduktion und schafft somit einen Orientierungsrahmen für die Subjektivierungsweise der Menschen, zum anderen erhöhen sich aber auch Perfektionsbestrebungen und können Abweichungssensibilität und eine erhöhte Fehlerintoleranz zur Folge haben. Die Allgegenwart von Vermessungsmethoden beschreibt Stefan Selke als neue Form der Biosozialität, die auf der Kontrolle durch Selbstregulierung und rationaler Diskriminierung ${ }^{21}$ beruht.

Auch wenn Stefan Selke versucht, sowohl Potenziale als auch Pathologien der disruptiven Lifelogging Technologien zu identifizieren, überwiegen bei ihm doch deutlich negative Facetten von Selbstvermessungspraktiken. Auf lange Sicht diagnostiziert er die Durchsetzung eines defizitorientierten Organisationsprinzips des Sozialen und fürchtet einen Machtzuwachs der Technologien, die durch die Bewertung über Mittelwerte und Kurven die dominanten Entscheidungsgeber werden könnten. Wenn sich die Normen von Selbstbeobachtungen zu einer permanenten Selbstüberwachung wandeln und die horizontale Kontrolle durch sichtbare Vernetzung

\footnotetext{
${ }^{21}$ Detaillierter zum Begriff der rationalen Diskriminierung: (Selke 2016b, 2020) Aber auch https://stefan-selke.de/ (zuletzt aufgerufen am 20.08.2020).
} 
der Daten allumfassend wird, steht für Stefan Selke die „Ära der Entscheidungsmaschinen“‘22 bevor, der es mit digitalem Verbraucherschutz und digitaler Medienbildung entgegenzuwirken gilt.

\section{Zwischenfazit}

Bisher konnten die dynamische Stabilisierung als Kennzeichen der Moderne herausgearbeitet werden und Folgen für die Subjekte abgeleitet werden. Beschleunigung, Flexibilisierung und Ökonomisierung lassen alte Bindung aufbrechen und seither feste institutionelle Grenzen verschwimmen zusehends. Immer mehr Bereiche des Lebens ordnen sich nach kapitalistischen Prinzipien und eine auf quantitative Steigerung ausgerichtete Optimierungslogik breitet sich auch in die subjektive und soziale Welt aus, die sich einem rationalen Kalkül bis dahin noch entziehen konnte.

Die dominante Subjektivierungsform der Moderne scheint das des Unternehmerischen Selbst, versinnbildlicht es doch eben diese Dynamiken. Der Mensch wird dazu aufgefordert, sich selbst zu erforschen, mithilfe dieses Wissens ständig zu wachsen, sich weiterzuentwickeln und sich selbst zur Lebendbewerbung zu formen. Nicht zuletzt Selbstvermessungspraktiken zeigen, wie fein die Mikrophysik der Macht geworden ist und neoliberale Narrative schreiben ihr Programm immer weiter in den menschlichen Körper ein. Bei den Subjekten lösen die steigenden Anforderungen einen Wunsch nach Ordnung, Struktur und Kontingenzreduktion hervor.

Dadurch entsteht laut Uwe Vormbusch ein „numerisch hervorgebrachtes, dargestelltes und kontrolliertes Subjekt"، dessen numerische Darstellung und Kontrolle durch Big Data und die Verbreitung informationeller Technologien noch zunimmt (Noji \& Vormbusch 2018, 31). Wie Stefanie Duttweiler zeigt, handelt es sich bei der Selbstvermessung um einen wechselseitigen Prozess der Bedeutungsgebung, bei dem die Daten und die Subjekte sich wechselseitig beeinflussen: ,[D]ie Daten werden in Form gebracht und mit Bedeutung versehen - und sie bringen die Einzelnen ,in Form', indem sie ihren Körperzuständen, ihren Alltagspraktiken oder ihren Zukunftsentwürfen Bedeutung verleihen“ (Duttweiler 2018, 273).

Zwei Dimensionen kristallisieren sich also bis hierhin heraus: Zum einen die Zunahme von Vergleichen und Bewertungen und zum anderen die Quantifizierung, die sich vor allem durch

\footnotetext{
${ }^{22}$ Möchte man sich näher mit Entscheidungsprozessen beschäftigen, an denen Algorithmen beteiligt sind, kann folgende Differenzierung hilfreich sein, auf die auch die deutsche Datenethikkommission zurückgreift: Demnach können Entscheidungen algorithmusbasiert, -getrieben, oder -determiniert sein und abgeleitet aus dieser Unterscheidung können sich rechtliche und ethische Fragestellungen anschließen, die hier allerdings unerwähnt bleiben müssen. Siehe: htps://datenethikkommission.de/gutachten/ (zuletzt aufgerufen am 03.09.2020).
} 
disruptive Technologien zusehends verbreitet. Bisherige Studien zu Selbstvermessung legen ihren Fokus dabei stark auf die zweite Dimension und behandeln vor allem die damit verbundenen Risiken. Zahlen wurden als Medium zur alltäglichen Selbstthematisierung gerahmt, die dadurch gleichzeitig ein Mittel zur Selbsterforschung bereitstellen und durch ihren scheinbar objektiven Charakter wichtige Ordnungsgeneratoren sind, die begleitet werden durch ein Gefühl der Sicherheit und Kontingenzreduktion.

Auch wenn die hier eingeführte Definition des Lifelogging theoretisch offen ist für qualitative Formen der Selbstthematisierung durch Daten, richtet Stefan Selke den Blick einseitig auf Selbstvermessungspraktiken in Form von Zahlen. Nimmt man Übersetzung des Lebens in Daten allerdings ernst, kann auch die aktive Social-Media-Nutzung unter den Begriff des Lifeloggings gefasst werden. Eine Analyse der digitalen Selbstdarstellung kann helfen, den heuristischen Begriff des Lifelogging einer kritischen Betrachtung zu unterziehen. Der Fokus wird damit weg von der Quantifizierung hin zu den digitalen Vergleichs- und Bewertungspraktiken verschoben. Zum Einstieg in den empirischen Teil der Arbeit wird zunächst der Forschungsgegenstand vorgestellt und der Forschungsstand aufgearbeitet. 


\section{Empirie: Methodisches Vorgehen}

Im folgenden Kapitel wird der methodische Aufbau der eigenen empirischen Untersuchung vorgestellt, woran sich eine Reflexion forschungsethischer Aspekte anschließt. Daran anknüpfend werden im Kapitel 5 die Ergebnisse dargelegt. Zuletzt findet eine Synthese und Interpretation der Ergebnisse aus beiden Teiluntersuchungen statt.

Beide Teiluntersuchungen teilen dabei die Auffassung, dass „Menschen auf der Grundlage ihrer Deutungen der sozialen Wirklichkeit handeln und diese Wirklichkeit nach bestimmten sozialen Regeln immer wieder neu interaktiv herstellen“ (Rosenthal 2015, 39). Als interpretative Methoden folgen sie den Prinzipien der Kommunikation und Offenheit, im Zentrum stehen also Ordnungssysteme des Alltags und die Strukturierung des Forschungsgegenstanden wird nicht aus der Theorie sondern den Subjekten heraus gebildet (vgl. ebd., 39f.). Im Anschluss an Alfred Schütz wird davon ausgegangen, dass die empirische Realität bereits von den in ihr lebenden Menschen gedeutet vorliegt und das Ordnung laufend reproduziert wird (vgl. Schütz 1971, 5ff.). Vor allem in der zweiten Teiluntersuchung stehen die alltäglichen Handlungen der Menschen im Mittelpunkt, wobei davon ausgegangen wird, dass sich soziale Interaktionen als Wechselspiel zwischen vorgegebenen Regeln und Normen und dem neu situativ Hergestellten gestalten (vgl. Goffman 1976).

\subsection{Methode I: Diskursive Interfaceanalyse}

In der ersten Teiluntersuchung wird eine diskursive Interfaceanalyse nach Mel Stanfill durchgeführt. Diese erlaubt es, durch die Untersuchung von Funktionalitäten, Menü-Optionen und dem Seiten-Layout eingebettete Machtstrukturen von Apps und anderer Software offenzulegen und damit die Subjektformierung zu zeigen, die auf Instagram an die Nutzenden herangetragen wird. Neue Medien werden als produktive Macht begriffen und zeigen, wie das Interface beides beinhaltet: die Reflexion und die Verfestigung sozialer Logiken. Indem man bei der Analyse dem foucaultschen Konzept produktiver Macht folgt, kann das Interface und die darin enthaltenden Annahmen als normativer bzw. ,richtiger“ Weg der Nutzung verstanden werden, ohne dabei davon auszugehen, dass diese Normen die Nutzung vollständig determinieren. Vielmehr geben sie gewissermaßen den Weg des geringsten Widerstandes vor, dem die Akteure folgen können - oder auch nicht (Stanfill 2015). Die zugrundeliegende Prämisse besteht also in der Annahme, dass durch die Nahelegung und höhere Wahrscheinlichkeit einer bestimmten Verwendungsweise die tatsächliche Nutzung beeinflusst und in gewissem Maße beschränkt wird 
(vgl. ebd., 1060). Das beinhaltet die Auffassung, dass gewisse Praktiken gewissermaßen gefördert, während andere eher zurïckgewiesen werden (vgl. Foucault 1990, 138).

Dem Prinzip der Diskursanalyse folgend wird nicht nach einer (vermeintlichen) Intention des Webdesigners geforscht, sondern vielmehr wird versucht, sich den Strukturen zu nähern, die dem Interface immanent sind und zur Produktion von Normativität beitragen (vgl. Stanfill 2015, 1061). Diskurse werden hier als Praktiken verstanden, die systematisch die Objekte formen, von denen sie sprechen, und damit einen Ort bilden, an dem Gedanken und Handlungen vor dem Hintergrund eines bestimmten Sets an Wissen und Annahmen darüber, was richtig und falsch ist, strukturiert werden (Foucault 1982, 49). Im Anschluss daran schreibt Stanfill:

„The underlying logic animating a design produces a ,correct‘ use even if different people produced various features - even if they were produced in apparent isolation from each other - and this happens in the context of the factors like consumer capitalism and intellectual property maximalism“ (Stanfill 2015, 1062).

Der foucaultschen Denkrichtung folgend muss also gefragt werden, wieso ein bestimmter Text, ein gewisses Design oder Layout erscheint und kein anderes (vgl. Foucault 1982, 27). Welche normativen Vorstellungen sind in das Design eingebaut und welche Konsequenzen ergeben sich daraus?

Die Affordanz ${ }^{23}$ bildet zur Beantwortung dieser Fragen einen wichtigen Zugang, um normative Ansprüche über die Funktion und die angemessene Verwendung ebenso zu ergründen wie das Bild eines „guten“ Nutzers oder einer „guten“ Nutzerin. Anstatt davon auszugehen, dass die Besucher*innen einer Website wissen, was sie möchten, und die Website versucht, diesen konkreten Wünschen gerecht zu werden, geht die diskursive Interfaceanalyse davon aus, dass gefragt werden muss, welche Wünsche der Nutzung überhaupt verfügbar oder auswählbar sind.

\footnotetext{
${ }^{23}$ Mit der Affordanz wird allgemein der Angebotscharakter von Artefakten beschrieben. So legt ein Sessel nahe, auf ihm zu sitzen, und eine Schere, mit ihr zu schneiden. Die Affordanz ist dabei aber nicht deterministisch zu verstehen, da zwar eine gewisse Aufforderung bzw. ein gewisses Angebot gemacht wird, das Artefakt aber immer auch anders verwendet werden kann, beispielsweise, wenn eine Schere zum Herausdrehen einer Schraube benutzt wird. Eines von vielen Beispielen für Geräte, die anders genutzt wurden als ursprünglich intendiert, ist die „Girl's Pager Revolution“: Als der Pager vor allem als Tool für Unternehmen eingeführt wurde, war das Gerät lediglich dazu gedacht, um Rückrufe anzufordern. Allerdings entwickelte sich vor allem unter jungen Japanerinnen der Pager zu einem komplexen Kommunikationsmedium. Erst später reagierten die Unternehmen auf diese Entwicklung, indem sie Textnachrichtendienste stärker in ihre Produkte integrierten (Fujimoto 2005; Katz \& Aakhus 2006). Obwohl die Pager also nicht dazu gedacht waren, längere Nachrichten zu verschicken, und dementsprechend die Oberfläche nicht wirklich dazu geeignet war, entstand ein Trend, der sogar einherging mit der Entwicklung einer eigenen Pager-Sprache, die vor allem junge Frauen zur Kommunikation über den Pager verwendeten.
} 
„Discursive interface analysis goes beyond function, examining affordances broadly - the features, but also what is foregrounded, how it is explained, and how technically possible uses become more or less normative through productive constraint.“ (Stanfill 2015, 1062) Die Strukturen der Website spiegeln wider, welche Annahmen der zukünftigen Nutzung bei der Programmierung berücksichtigt wurden. Damit werden diese zum normativen Anspruch, wie Nutzer*innen sich in der späteren Realität verhalten sollen. In der Designphase wird so das Bild eines perfekten Nutzers bzw. einer perfekten Nutzerin implizit mitgeschaffen, zumindest als Idealtyp. Man darf dabei nicht müde werden zu betonen, dass dieser Ansatz nicht aus einer technik-deterministischen Perspektive argumentiert: Die Affordanz setzt dem Benutzer oder der Benutzerin gewisse Grenzen, die den Möglichkeitsraum abstecken. Die Nutzungsoberfläche macht gewisse Handlungen wahrscheinlicher und möglicher als andere, wie sich der Mensch aber in der konkreten Situation verhält, ist weder garantiert noch vollständig vorherbestimmt.

\section{Funktionale, kognitive, sensorische Affordanz}

Für die anschließende Analyse ist es sinnvoll, sich dem Konzept der Affordanz bzw. des Angebotscharakters noch eingehender zu widmen. Nach Hartson (2003) können vier verschiedene Affordanztypen unterschieden werden: die kognitive, physikalische, sensorische und die funktionale Affordanz. Da in dieser Arbeit das virtuelle Interface betrachtet wird, spielt die physikalische Affordanz nur eine untergeordnete Rolle in der Analyse und wird an dieser Stelle nicht näher erläutert.

Als ersten Schritt kann man sich also die simple Frage stellen, welche Funktion eine Seite oder App hat: Was kann man damit tun? Was kann man sehen und selbst auf der Seite produzieren? Kann man etwas downloaden oder speichern? Die funktionale Affordanz produziert Normen, indem sie etwas erlaubt oder nicht erlaubt. Nutzer*innen wird dadurch nahegelegt, sich auf eine bestimmte Art und Weise zu verhalten(vgl. Stanfill 2015, 1063). Darunter fallen alle Handlungsmöglichkeiten, die die App bietet, ob man beispielsweise Bilder erstellen kann, Text eingeben, ein Spiel spielen oder ein Kreuzworträtsel lösen kann.

Die kognitive Affordanz hilft dem oder der Nutzer*in sich für eine Handlung zu entscheiden. Über Menüoptionen und verschiedene Features oder Benutzerfreundlichkeit und darüber, wie bestimmte Aspekte mehr oder weniger auffällig gestaltet sind, wird die Informationsverarbeitung erleichtert. Die diskursive Natur wird hier deutlicher, da dadurch die Nutzung eng ver- 
knüpft ist mit der sozialen Bedeutungsproduktion. Die kognitive Affordanz verweist auf Labeling, Namensgebung, Selbstbeschreibung und Slogans. Eine Seite als „offiziell“ zu bezeichnen, schafft zum Beispiel den Eindruck von Legitimität, während die Bezeichnung „Fan Page“ die Bedeutung verschiebt, auch wenn der Inhalt möglicherweise ähnlich ist. Während auf der offiziellen Website von Instagram der Slogan erscheint „Näher an den Menschen und Dingen die du liebst [Hervorhebung im Original; M.R.]“24, erscheint auf der TikTok-Website „Make your Day - Real People. Real Videos ${ }^{\text {“25 }}$. Beide Social-Media-Seiten sind relativ ähnlich aufgebaut, schon der Slogan macht aber einen anderen Fokus deutlich und adressiert eine bestimmte Personengruppe als Nutzer*innen. Wie ein Menü oder eine Überschrift gestaltet und bezeichnet wird, ist bedeutsam als Definition darüber, was der oder die Nutzer*in durch die Auswahl dessen tut (vgl. ebd., 1063f.). Ein weiteres Beispiel sind die Optionen, die bei der Einrichtung des Profils zur Verfügung stehen: Muss man das eigene Geschlecht angeben? Gibt es dafür genau zwei Auswahlmöglichkeiten?

Den dritten Typ bildet die sensorische Affordanz und kommt einer ästhetischen Analyse des Interface wahrscheinlich am nächsten. Bewegt sich viel oder ist die App/Seite eher ruhig gestaltet? Gibt es ein einheitliches Farbschema? Ist das Design aufgeregt oder ruhig und organisiert? Denn es macht durchaus einen Unterschied, ob auf einer Seite viele Werbeanzeigen mit Farbe, Bewegung und Geräuschen versuchen, Aufmerksamkeit zu generieren, oder ob sie eher als ruhig und klar beschrieben werden kann. Während das eine einen sehr kommerzialisierten Eindruck hinterlässt, erwirkt das zweite eher den Eindruck von Verlässlichkeit und Professionalität. Dadurch werden Annahmen über Inhalt und Zielgruppe einer Seite noch einmal verstärkt (vgl. ebd., 1064). Es macht beispielsweise einen großen Unterschied, ob man die Website der Bild oder der Süddeutschen oder Frankfurter Allgemeinen Zeitung aufruft. ${ }^{26}$ Zur sensorischen Affordanz zählt darüber hinaus, wie eine Seite angeordnet ist und wo welche Inhalte platziert sind. Dinge, die links oben erscheinen, erhalten zum Beispiel (für westlich sozialisierte Betrachter*innen) mehr Aufmerksamkeit als etwas, das rechts unten erscheint. Außerdem erreichen Inhalte, die auf der Startansicht der App oder Seite erscheinen, mehr Aufmerksamkeit als Inhalte, die erst durch Scrollen erscheinen. Stanfill verweist darauf, dass diese Art der Affordanz vielleicht auf den ersten Blick weniger diskursiv aufgeladen erscheint, die Tatsache aber, dass etwas hervorsticht oder in den Vordergrund gerückt wird, Wertigkeit und Bedeutung in gewisse Aspekte legt, und in der Hand der Webdesigner liegt (vgl. ebd., 1064).

\footnotetext{
${ }^{24}$ https://about.instagram.com/ (zuletzt aufgerufen am 17.06.2020).

${ }^{25} \mathrm{https}: / /$ www.tiktok.com/de/ (zuletzt aufgerufen am 17.06.2020).

${ }^{26} \mathrm{https} / / /$ www.bild.de/, https://nl.faz.net/ und https://www.sueddeutsche.de/ (zuletzt aufgerufen am 17.06.2020),
} 
Zusammengefasst werden also über die Betrachtung des Angebotscharakters, der in das Interface von Instagram eingebettet ist, Rückschlüsse auf normative Ansprüche und ein gewünschtes Verhalten möglich. Diese Teilanalyse zielt demnach auf die Rekonstruktion der Subjektformierung auf Instagram ab. Wie gestaltet sich ein ,gutes“ Subjekt auf Instagram, welche Handlungsweisen werden den Akteuren nahegelegt und welche Normen lassen sich daraus ableiten?

\subsection{Methode II: Online-Ethnografie}

Die zweite Teiluntersuchung begibt sich auf die Ebene der Subjektivierungsweise. Vor dem Hintergrund der eingehenden Erforschung des Interface, stellt sich dich Frage, wie die Menschen sich tatsächlich auf Instagram bewegen: Wie handeln sie innerhalb der Möglichkeiten, die durch die App vorstrukturiert werden? Wie werden Potenziale ausgeschöpft oder wie werden die normativen Vorgaben bewusst oder unbewusst umgangen? Um diese Fragen zu beantworten, gibt eine ethnografische Vorgehensweise die richtigen Werkzeuge an die Hand.

Der empirische Teil der Arbeit orientiert sich an den Richtlinien der interpretativen Sozialforschung und wurde als exploratives Design konzipiert. Hubert Knoblauch sieht in der Ethnografie ein großes Potenzial und hebt im Anschluss an die Kritik der Konversationsanalyse die Vorteile eines offenen Forschungsvorgehens hervor: „Denn angesichts einer sich rasant wandelnden Gesellschaft können Untersuchungen ihrer Struktur keineswegs mit vorgefertigten Mustern verfahren“ (vgl. knoblauch 2002, 134). Darüber hinaus ist es möglich, durch die Beobachtungen auch Handlungspraktiken aufzudecken, die nicht „sagbar“, also nicht in einem Interview erfragbar, wären. Es wird davon ausgegangen, dass die Instagramnutzung zu einem großen Teil auf routinierten Handlungen und Entscheidungen beruht, die schwer verbalisierbar sind, weshalb der ethnografische Zugang hier mehr Möglichkeiten der Untersuchung von Lifeloggingpraktiken auf Instagram bietet. Das Forschungsdesign folgt also dem Prinzip der Offenheit gegenüber dem Untersuchungsgegenstand, um zugrundeliegende Sinnstrukturen zu rekonstruieren. Deshalb werden keine vorgelagerten Hypothesen durch die Daten getestet, sondern in die Analyse an der empirischen Wirklichkeit ausgerichtet (vgl. Kleemann, Krähnke \& Matuschek 2009, 19).

Nun ist bekannt, dass die Ethnografie im traditionellen Sinne sich sehr ausführlich mit Gemeinschaften auseinandersetzt. Durch den Eintritt des Forschers oder der Forscherin in diese Gemeinschaft und die aktive Teilnahme am Leben derselben bieten sich Einblicke, die tiefer gehen als bloße Befragung oder Beobachtung von außen. Auf den ersten Blick erscheint es deshalb befremdlich, eine ethnografische Herangehensweise für die Untersuchung online stattfindender Interaktionen zu wählen. Allerdings wurde der Begriff der Netnografie bereits in den 1990er 
Jahren von Kozinets geprägt. Durch die Verbreitung des Internets und in seiner grenzüberschreitenden Eigenschaft war es naheliegend, sich die Vielfalt des Mediums und der darin enthaltenen Daten für die Forschung (damals vor allem der Marketing- und Konsumforschung) nutzbar zu machen (vgl. Kozinets 1998). Schon früh beschäftigten sich Forschungsarbeiten mit internetvermittelter Identitätsbildung oder Konsumkultur (vgl. Beckmann \& Langer 2009, 221). Abseits klassischer Konsumforschung haben sich auch Soziolog*innen bereits online ins Feld gewagt. Die Arbeiten versuchen dabei meist, ausgehend von einer klassischen ethnografischen Fragestellung, auch die Kommunikation und Interaktion der untersuchten Gemeinschaft über das Internet miteinzubeziehen, oder beschäftigen sich mit Online-Communities zum Beispiel auf WorldofWarcraft oder anderen Online-Spielen (Boellstorff et al. 2012). Die Vorgehensweise in dieser Arbeit unterscheidet sich insoweit von bisherigen netnografischen Studien, das erstens keine abgeschlossene Community als Untersuchungsgegenstand gewählt wurde und zum zweiten in der ausschließlichen Beobachtung virtuell stattfindender Interaktionen. Es wäre sicherlich spannend gewesen, die Untersuchung auch auf „face-to-face“-Situationen auszuweiten und aktiver Teil einer gewissen Peer-Group zu werden. Durch die Ausbreitung des CoronaVirus und den Lockdown konnte eine solche Vorgehensweise leider nicht umgesetzt werden. Die Anfänge der Arbeit liegen im März 2020 also in einer Zeit der Kontaktbeschränkung und des Social-Distancing. Desto mehr kann jedoch die momentane Lage als Chance gesehen werden, sich ganz auf die virtuellen Geschehnisse einzulassen. Wie bereits skizziert verbringen Menschen immer mehr Zeit online und es ist absehbar, dass dieser Trend sich in den nächsten Jahren fortsetzen wird. Die Fokussierung auf Handlungsweisen innerhalb Instagrams wird also nicht als Beschränkung, sondern vielmehr als Möglichkeit gesehen, die dort stattfindenden Interaktionen, Praktiken und Kommunikationsvorgänge als vollwertige Situationen zu betrachten und nicht wie bisher oft hinter ,face-to-face“-Situationen zurückzustellen. ${ }^{27}$

\section{Zugang}

Der Zugang zum Feld erfolgte zunächst durch die eigene Nutzung. Durch existierende Forschungsliteratur zum Thema, gezielte und ungezielte Hashtagsuche und intensives Browsen im

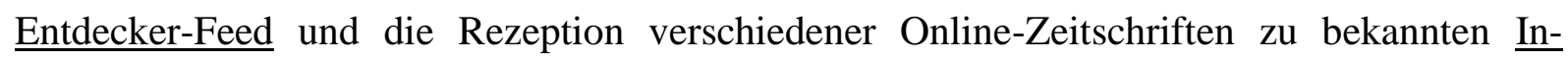
fluencer*innen wurde ein großer Pool an abonnierten Profilen geschaffen und darüber hinaus versucht, das eigene Netzwerk auszuweiten. Die eigene Nutzung und Einfühlung in die App wird als wichtiger Bestandteil für das Verständnis der später erhobenen Daten betrachtet (vgl. Krüger \& Spilde 2019). Vor Beginn der Arbeit wurde die App von der Autorin zwar bereits

\footnotetext{
${ }^{27}$ Siehe dazu auch Knorr-Cetina 2012a, 2012b.
} 
genutzt, war allerdings nicht aktiv im Sinne von eigenen Posts oder Interaktionen mit anderen Profilen, weswegen eine tiefere Auseinandersetzung nötig war. Da viele der so in den Blick genommenen Profile fast ausschließlich öffentlich waren, war der Zugang zunächst sehr niederschwellig möglich. Durch einen einfachen Klick können die Profile abonniert werden, um über neue Inhalte informiert zu werden und diese in der Timeline angezeigt zu bekommen. Die bei der Recherche gefundenen Profile wurden dabei zwar vermeintlich zufällig entdeckt, beruhen allerdings zwangsläufig auf Vorschlägen, die die App aufgrund früherer Suchanfragen vorschlägt, ein gewisser Bias kann daher nicht ausgeschlossen werden. Damit kann zwar kein repräsentatives Bild gezeichnet, dennoch aber eine möglichst große Bandbreite in den Daten widergespiegelt werden. In einem nächsten Schritt wurden einzelne Profile ausgewählt und teilweise über die angegebene E-Mail-Adresse, meist aber über die DM-Funktion angeschrieben und nach Interesse an Teilnahme der Untersuchung gefragt. Außerdem wurden die potenziellen Untersuchten über die Art der Datenerhebung aufgeklärt. Das Anschreiben der einzelnen Personen wurde darüber hinaus als Chance betrachtet, um in direkten Austausch zu treten und Möglichkeiten und Raum für Interviews oder Nachfragen zu schaffen.

Die Auswahl der Probanden wurde als theoretisches Sampling konzipiert und dabei versucht sowohl minimal als auch maximal kontrastierende Fälle auszuwählen (vgl. Rosenthal 2015). Da die Bandbreite an Nutzern und Nutzerinnen auf Instagram sehr weit gefächert ist, kann der Anspruch nicht sein, alle möglichen Nutzertypen zu identifizieren und im Datenmaterial abzubilden. Das Aufnehmen möglichst unterschiedlicher Profile ermöglicht es aber, vielfältige Facetten zu zeigen und der Diversität der Nutzenden trotz des kleinen Datenkorpus gerecht zu werden. Im Anschluss an die Forschung zur Selbstvermessung, die sich oft im Kontext um den Körper bewegt, wurde vor allem nach Menschen gesucht, die sich ebenfalls mit diesem Thema auseinandersetzen. Deshalb wurden zum einen Fitness- und Lifestyle-Blogger*innen mit einer möglichst hohen Reichweite angeschrieben und zum anderen nach Menschen gesucht, die dem gängigen (Schönheits-)Ideal der schlanken, fitten, gesunden und attraktiven Frau in irgendeiner Form nicht entsprechen. Ursprünglich wurde angestrebt, auch mindestens einen männlichen Nutzer in den Daten zu repräsentieren, leider kamen aber trotz zahlreicher Anfragen keine positive Rückmeldung. Auch über das Schneeballsystem konnte kein Mann für die Untersuchung gewonnen werden. Die Tatsache wird aber nicht als Schwäche bewertet, da zum einen Frauen die dominante Nutzergruppe auf Instagram darstellen und zum anderen ist es besonders spannend, Körperpraktiken weiblicher Nutzerinnen in den Blick zu nehmen, da ihre Körper in unserer Gesellschaft stärker durch die Brille von Schönheit und Formung betrachtet werden. Es 
ist wichtig, diesen Aspekt in der Arbeit anzuerkennen und bei der Auswertung zu berücksichtigen. Ein letztes Auswahlkriterium stellte die Aktivität der Profile dar, aufgrund des onlineethnografischen Zugangs können nur aktiv generierte Inhalte und öffentlich sichtbare Interaktionen der Nutzer*innen erfasst werden, weswegen bei der Probandenauswahl darauf geachtet wurde, dass die Profile regelmäßige Content produzieren.

\section{Datenerhebung}

Während der Feldphase wurden die Daten zum einen mittels Screenshots auf dem mobilen Endgerät und als Desktopaufnahmen gespeichert. Auch Kommentare konnten so archiviert werden. Da die Stories meist aus kurzen Videos bestehen, wurden bewegte Inhalte durch einen sogenannten Screen-Recorder festgehalten. Diese Methode der Datenerhebung hat sich bereits in meiner Bachelorarbeit bewährt, bei der es durch die Verwendung des Screen-Recorders möglich war, die Nutzungsvorgänge während der Snapchat-Nutzung für eine soziologische Untersuchung zugänglich zu machen. Die App ist kostenlos über den PlayStore verfügbar und ist während der Aufnahme nur als kleines Symbol am Bildschirmrand sichtbar. Die App zeichnet alle Aktivitäten auf dem Bildschirm auf und über das Mikrofon des Smartphones werden auch Geräusche eingefangen. Nach Beenden der Aufnahme wird eine Videodatei im mp4-Format erstellt. Dadurch, dass Fotos und Videos, von denen ein Screenshot erstellt wird, keine Benachrichtigung auslösen, sind die erhobenen Daten „natürlich“, also nicht durch den Forschungskontext beeinflusst. Da Stories lediglich 24 Stunden sichtbar sind und auch teilweise sehr häufig Stories erstellt wurden, wurde über einen Zeitraum von zwei Wochen morgens und abends je eine solche Aufnahme von jedem untersuchten Profil erstellt. Damit wurden alle Stories in diesem Zeitraum archiviert, danach wurden nur noch Screenshots einzelner Story-Posts erstellt, um den Datenkorpus nicht zu überfrachten. Die während der Feldphase erstellen regulären Post sind im Feed der jeweiligen Personen gespeichert und mussten deshalb nicht zusätzlich mit einem Screen-Recorder festgehalten werden. 


\subsection{Forschungsethik}

Für die vorliegende empirische Untersuchung bilden Überlegungen zu einer angemessenen Herangehensweise, die auch forschungsethischen Gesichtspunkten gerecht wird, einen essenziellen Bestandteil. Die erste Teiluntersuchung gestaltet sich diesbezüglich zwar unproblematisch, umso notwendiger sind forschungsethische Überlegungen jedoch im zweiten Teil. Eine ethnografische Untersuchung ist prinzipiell so vielfältig wie menschliche Interaktionen selbst. Die Aufgabe des Forschers oder der Forscherin besteht deshalb notwendigerweise darin, sensibel, mit Achtung und mit Taktgefühl in die Situation hineinzugehen. Durch die Nähe zum Feld und die Erhebung teils sehr persönlicher Daten aus dem alltäglichen Leben der Menschen muss der oder die Forscher*in Sorge für die Untersuchten tragen und gewährleisten, dass ethische Grundregeln eingehalten und gewährleistet werden können (vgl. Unger, Narimani \& M’Bayo 2014). Da die Forschung von der Offenheit der Untersuchten lebt und nur durch deren Vertrauen möglich gemacht wird, ist es wichtig, dieses nicht auszunutzen. Nicht zuletzt aufgrund der ungleichen Machtverteilung zwischen Untersuchtem und Untersuchenden ist es von großer Bedeutung, die Rücksicht und das Wohlbefinden des Untersuchten fest im Forschungsdesign zu verankern. In der zweiten Teiluntersuchung werden nach Einholen des Einverständnisses über einen gewissen Zeitraum Screenshots und Aufnahmen täglicher Posts und Stories erstellt. Vor allem die Stories bestehen aus sehr sensiblen Daten, da sie meist sehr persönlich sind und in ihrer Struktur nicht zur Archivierung ausgelegt sind. Nach 24 Stunden löschen sich die dort geposteten Inhalte selbstständig und sind dann für die Community nichtmehr sichtbar. Die Archivierung derselben ist also durchaus kritisch und bedarf der Abstimmung mit dem Untersuchten, die in für die Arbeit eingeholt wurde. Die Machtasymmetrie wurde versucht zumindest zu einem Teil auszugleichen, indem das Profil der Forscherin für die Zeit der Datenerhebung öffentlich war und in der Profilbeschreibung die eigene Position deutlich gemacht wurde. Auch das Angebot in der Bio, bei allen Fragen zur Verfügung zu stehen, und die Übermittlung des eigenen Profilnamens sollten $\mathrm{zu}$ einem ausgewogeneren Informationsgehalt beitragen. Vertrauen und gegenseitiger Respekt bildet damit einen unverzichtbaren Bestandteil der zweiten Teiluntersuchung (vgl. Boellstorff et al. 2012, 130). 


\section{Informed Consent}

Vielleicht als wichtigste Vorarbeit zur eigentlichen Untersuchung ist das Einholen des Einverständnisses der Nutzenden zu betrachten. Bei der Recherche fiel auf, dass verschiedene empirische Arbeiten sehr unterschiedlich mit den erhobenen Daten umgehen. Währen Schreiber und Kramer Usernamen und Bilder in ihren Veröffentlichungen unkenntlich machen und Einverständnis einholen (vgl. Kramer \& Schreiber 2016), verzichten andere Studien auf derartige forschungsethische Aspekte und benutzen Daten ohne Einverständnis oder Anonymisierung. Letztere Praxis wird in dieser Arbeit als höchst kritisch eingestuft, da sie gängigen forschungsethischen Ansprüchen (vgl. Unger, Narimani \& M’Bayo 2014) nicht entspricht und dadurch entstehende Probleme ignoriert.

Es ist zwar richtig, dass diese Menschen zumindest zu einem Teil Personen öffentlichen Lebens darstellen und sich nicht scheuen private Badezimmerselfies mit einer großen Community und potenziell nicht absehbarer Reichweite teilen. Trotzdem handelt es sich hier um Menschen, deren Gefühle und Meinungen berücksichtigt werden sollten. Deshalb fand schon relativ früh die Entscheidung statt, in den Austausch mit den Menschen zu treten, die Teil der Untersuchung bilden sollten. Dafür wurden potenzielle Teilnehmer*innen teilweise über die Nachrichtenfunktion auf Instagram, teilweise per E-Mail kontaktiert und über das Forschungsvorhaben ausführlich informiert. Anschreiben, die keine Antwort erhielten, wurden ebenso nicht in die Datenpool aufgenommen, wie explizite Ablehnungen. ${ }^{28}$ Um darüber hinaus Sichtbarkeit der eigenen Rolle und Interessen zu schaffen, wurde das private Profil angepasst und darüber informiert, dass sich dahinter eine Soziologin befindet, die sich mit der Fragestellung beschäftigt, wie Menschen sich auf Instagram darstellen. Der Text wurde auf Englisch verfasst, um auch mögliche sprachliche Barrieren zu überwinden. Um den informed consent als dynamischen und dialogischen Prozess zu gestalten, der nicht bei der anfänglichen Einverständniserklärung stehen bleibt, waren über die App Nachfragen jederzeit möglich. Da der Name des für die Arbeit verwendeten Profils bekannt war, konnten die Untersuchten jederzeit nachvollziehen, welche Stories angesehen wurden und welche nicht.

\footnotetext{
${ }^{28}$ Eine ausdrückliche Ablehnung gab es tatsächlich nur bei einem Profil, die Regel waren schlicht keine Antworten auf die Anfrage. Da auch sehr bekannte Influencer*innen mit sehr hoher Followerzahl angefragt wurden, ist die Wahrscheinlichkeit groß, dass die Nachricht gar nicht erst gelesen wurde, sondern unterging. Anfangs wurde das Anschreiben sehr neutral und so knapp wie möglich gehalten. Allerdings war nach einigen Versuchen klar, dass diese Art des Anschreibens sich nicht mit den Konventionen auf Instagram deckt. Die gegenseitige Bestätigung und Lob scheinen zur Etikette des gegenseitigen Austausches zu gehören. Deshalb wurde die Anfrage angepasst und enthielt zunächst Bewunderung oder positive Anerkennung über die Posts der oder des Anderen (bspw. „Danke für deinen tollen Content und dass du dein Leben so offen zeigst $<3$ “). Diese angepasste Form führte zu höherer Resonanz.
} 


\section{Privatheit in Zeiten von Big Data}

Obwohl so gewährleistet war, dass das Verhältnis zwischen Untersuchten und Forscherin so ausgewogen war wie möglich, konnten nicht alle Aspekte rund um Privatheit und Anonymität vonseiten der Forscherin kontrolliert werden. Die derzeitige digitale Umgebung im Allgemeinen und Instagram im Besonderen schließt vielfältige Akteure und Variablen mit ein, die vom eigenen Standpunkt im Feld aus nicht kontrolliert werden können. Es existieren beispielsweise keine Information über die Daten, die während der Recherche oder auch während der Feldphase unbewusst erzeugt wurden, welche Algorithmen im Hintergrund abliefen oder welche Rückschlüsse aus dem eigenen Verhalten auf Instagram möglicherweise gezogen wurden. Wie atypisch die Nutzung im Zuge der Arbeit war, wird durch den Entdecker-Feed deutlich. So dachte der Instagram Algorithmus kurzzeitig, das Profil der Forscherin sei eine russischsprachige Person und zeigte fast nur Beiträge auf kyrillisch an. Buchanan formuliert in diesem Kontext treffend:,,The plethora of data streams in constant production feeds into big data analytics, simultaneously adding value to user experiences while undermining any notion of individual privacy, autonomy, or consent“" (Buchanan 2019, 378).

Eine vollständige Privatheit der Untersuchten im Netz kann evidenterweise nicht vonseiten der Forscherin gewährleistet werden. Die angestellten Überlegungen zu Privatheit und Umgang mit den sensiblen Daten beschränkt sich also auf die Verwendung in der Arbeit selbst und das Teilen der erhobenen Daten mit Kommiliton*innen (im Rahmen eines Forschungskolloquiums) sowie den Betreuenden und anderen möglichen Leser*innen der Arbeit. Profilnamen werden im Folgenden ebenso geschwärzt wie Informationen, die Rückschlüsse auf den Namen oder Wohnort der Untersuchten zulassen könnten. Da niemand einen Wunsch nach Unkenntlichmachung der Bilder äußerte, kann im Folgenden der Content unanonymisiert abgebildet werden.

Ein letzter Aspekt bildet die Möglichkeit bei Interesse die Arbeit an die Untersuchten zu versenden. Dieses wurde von einer Probandin geäußert und ihr deshalb nach der Abgabe zugesendet. 


\section{Digitale Selbstdarstellung auf Instagram}

In diesem Kapitel wird die Social-Media-App Instagram als Untersuchungsgegenstand dieser Arbeit vorgestellt und die dazu relevante Literatur knapp erläutert. Danach folgt die Darstellung der beiden Teiluntersuchungen. In Kapitel 5.4. werden die Ergebnisse aus diskursiven Interfaceanalyse und der Online-Ethnografie zusammengeführt und interpretiert.

\subsection{Beschreibung des Untersuchungsgegenstandes}

Instagram gehört mittlerweile zu den meist genutzten Social-Media-Apps und war bereits kurz nach seiner Einführung 2010 das soziale Medium mit dem schnellsten Zuwachs an Nutzerzahlen (vgl. Sheldon/Bryant 2016: 89). Durch den sehr einfachen Aufbau und die Möglichkeit, Bilder unkompliziert in der App zu erstellen, zu editieren und auf der Plattform zu teilen, entstand die heutige Beliebtheit der App. In der Forschung wird Instagram überwiegend als Ort der Selbstdarstellung verhandelt. Neben Studien, die sich direkt mit der App beschäftigen, sind in diesem Zusammenhang auch Auseinandersetzungen mit neuen Arten des Fotografierens und ihrer Rolle als identitätsstiftende Praktiken von Interesse. In psychologischen und bildungswissenschaftlichen Studien fanden schon früh Untersuchungen zu verschieden Formen der SelbstRepräsentation mittels Social-Media und Nutzungsmotiven statt. Während im psychologischen Diskurs vor allem eine Auseinandersetzung mit Narzissmus, Selbstbewusstsein und Selbstvermarktung erfolgt, drehen sich pädagogische Debatten stärker um die Rolle, die Instagram in der Identitätsbildung junger Erwachsender einnimmt (Bakhshi, Shamma \& Gilbert 2014; Moon et al. 2016; vgl. Schreiber \& Kramer 2016; Sheldon \& Bryant 2016). In kommunikations- und medienwissenschaftlichen Arbeiten wurden in der Vergangenheit Themen wie Sichtbarkeit und Ruhm oder Bekanntheit unter anderem im Vergleich zu klassischen Medien verhandelt, aber auch die Konstruktion und Reproduktion von Geschlecht und Geschlechtsstereotypen wurde bereits untersucht (Abidin 2016; Caldeira, Ridder \& van Bauwel 2018; Manovich 2017; vgl. Marwick 2015).

Schon bevor Instagram den Markt eroberte, entwickelten sich aus persönlichen Blogs Seiten, die mit heutigen Influencer-Profilen vergleichbar sind. Auf den sogenannten Lifestyle-Blogs entwickelten sich bekannte Blogger*innen zu wirkungsvollen Werbeträger*innen. In diesem Kontext wurden Blogger*innen als voicy consumers schon 2005 relevant für Unternehmen und deren Marketing. Bereits in den frühen Blogs war durch eine Kommunikationsfunktion eine Interaktion über die täglichen Posts der Blogger*innen möglich. Eben über diese persönliche, glaubhafte und interaktiv eingebettete Inszenierung des Alltags gelang es, bestimmten Objekten 
Wert zu verleihen und im Mantel des consumer review posts auch gesponserte Artikel zu bewerben. Der Erfolg dieser Art des Marketings begründet sich nicht zuletzt durch den Aufbau von Nähe und Intimität und durch die Illusion einer realen ,face-to-face“-Beziehung, da die Blogger*innen ihre Fans meist direkt ansprechen und eine Art para-soziale Beziehung aufbauen. Zwar erreichten die Lifestyle-Blogs keine globale Öffentlichkeit, sprachen aber gezielt lokale Zielgruppen an. Über die Professionalisierung des consumer review posts fand also eine Transformation des vormals persönlichen und privaten Blogs zum ökonomisierten LifestyleBlog statt (vgl. Hopkins 2019). Wie sich später zeigen wird, schreibt Instagram diese Entwicklung weiter, da tägliche Alltagserzählungen und Produktplatzierungen in den Profilen der Influencer*innen fast vollständig verschwimmen.

Deskriptiv können quantitative Umfragen und Big-Data-Analysen Aufschluss über die Verbreitung, Nutzertypen und Motive geben. So bilden junge Frauen die Hauptzielgruppe für Instagram. Das Nutzungsverhalten konzentriert sich zeitlich auf das Wochenende und den Nachmittag bzw. Abend. Diese Erkenntnis deckt sich auch mit Empfehlungen in verschiedenen YouTube-Ratgebern. So wird neben den Inhalten an sich auch die Zeit des Postens geplant und angehenden Influencer*innen wird nahegelegt, Content abends und am Wochenende zu posten, um eine höhere Engagementrate zu erhalten. Neben der Zeit des Hochladens beobachten Forscher*innen ein ,rich get richer“-Phänomen, also eine gewisse sich selbstverstärkende algorithmusbasierte Aufmerksamkeit, wenn Inhalte mit vielen Likes noch mehr Likes erhalten (vgl. Araújo et al. 2014, 5). Zurückzuführen ist das vermutlich auf die Funktionsweise der Algorithmen hinter Instagram, die Posts mit einer hohen Fluktuation präferiert im Feed

von User*innen anzeigt. Auf Basis von Big-Data-Analysen kann allgemein gesagt werden, dass Fotos, auf denen Gesichter abgebildet sind, deutlich mehr Likes und Kommentare erhalten, unabhängig von der allgemeinen Reichweite und der Aktivität. Menschliche Gesichter sind also neben den anderen Modalitäten und Kommunikationsmöglichkeiten auf Instagram ein kraftvoller Kanal nonverbaler Kommunikation (vgl. Bakhshi, Shamma \& Gilbert 2014, 965).

Als Nutzungsmotive arbeiten Sheldon und Bryant (2016) vor allem den Überwachungs- bzw. Wissensaspekt heraus. So wird Instagram von vielen genutzt, um über den Alltag, aber auch über relevante soziale Beziehungen ihrer abonnierten Personen informiert zu werden. Spiegelbildlich dazu bildet die Dokumentation ein zentrales Motiv bei der Nutzung. Außerdem zeigt sich, dass auch Aspekte wie Coolness und Kreativität die Nutzung von Instagram motivieren (vgl. Sheldon \& Bryant 2016, 92). 
Es ist auffällig, dass vor allem Frauen zu den Nutzenden zählen. Damit sind es also auch vor allem Frauen, für die es attraktiv scheint, sich und ihren Körper online zu präsentieren und zu inszenieren. Dass eine Plattform, die ihren Fokus stark auf das Visuelle legt, ein vermehrt weibliches Publikum anzieht, ist keinesfalls Zufall und hängt eng mit Diskursen um den weiblichen Körper zusammen. Diese Facette Instagrams ist überaus bedeutsam und verrät viel über die ungebrochene Bedeutung weiblicher Attraktivität. Mit der Selbstdarstellung von Weiblichkeit auf Instagram und den politics of gender representation beschäftigt sich beispielsweise Sofia Caldeira und hebt vor allem die diffuse Macht hervor, die von den User*innen wechselseitig ausgeübt wird (vgl. Caldeira, Ridder \& van Bauwel 2018). Die geschlechtsspezifische SelbstPräsentation ist dabei eingebettet in komplexe Aushandlungsprozesse und verbindet widersprüchliche Diskurse. „They emphasize the importance of attentive curation in self-representation practices, acknowledging the influence of social and aesthetic conventions of photographable and instagrammable“ (Caldeira, Ridder \& van Bauwel 2020, 1). Dabei bewegen sich die Userinnen auf Instagram in einem Spannungsverhältnis zwischen dem Wunsch nach Authentizität und den Ansprüchen der Instagramability (vgl. ebd., 3). ${ }^{29} \mathrm{Um}$ den Rahmen der Arbeit nicht zu sprengen, kann an dieser Stelle nur auf die Relevanz der Auseinandersetzung mit diesem Thema verwiesen werden, ohne es selbst detailliert auszuarbeiten.

Ebenfalls sehr spannend für diese Arbeit ist ein Artikel von Sascha Oswald zur Subjektformierung und Evaluationsfunktion auf Instagram. Anhand von Interviews mit Instagram Nutzer*innen betrachtet er vor allem die Funktion von Likes und Kommentaren und die damit verbundenen Subjektvorstellungen sowie die Folgen für Handlungs- und Deutungsmuster der Nutzenden. Die Fragestellung ähnelt in manchen Aspekten dem leitenden Interesse dieser Arbeit und geht ebenfalls von Hartmut Rosas Modell der dynamischen Stabilisierung aus (vgl. Oswald 2019). Allerdings unterscheidet sich der theoretische und methodische Zugang vom hiesigen, da Sascha Oswald seine Analyse bei Kathi Knorr-Cetinas Konzept der skopischen Medien ${ }^{30}$ beginnt und mit qualitativen Interviews arbeitet. An dieser Stelle soll nur kurz auf grundlegende Erkenntnisse dieser Studie eingegangen werden, um später in der eigenen Analyse einige Aspekte aufzugreifen und die erhobenen Daten durch Ergebnisse aus den dort durchgeführten Interviews zu validieren und zu ergänzen.

\footnotetext{
${ }^{29}$ Auf den Begriff der Instagramability, oder Instagramabilität wird in Kapitel 5.7. genauer eingegangen.

${ }^{30}$ Skopische Medien können als Technologien definiert werden, die physisch nicht präsente Ereignisse oder Handlungen in Situationen projizieren, in denen sie sensorisch wahrnehmbar sind. Sie sind damit in der Lage die klassische face-to-face Situation sowohl informativ als auch interaktiv zu erweitern. Näheres zum Begriff der skopischen Medien siehe Knorr-Cetina 2012a.
} 
Dadurch, dass Instagram als Beispiel eines skopischen Mediums gerahmt wird, geht Sascha Oswald der Frage nach, wie sich Interaktionen und Selbstbezüge im digitalen Raum durch Kommunikationsplattformen strukturieren. Durch das Echtzeit-Feedback in Form und Likes und Kommentaren entstehen synthetische Situationen, die neue Formen des Publikumsbezugs und eine Umwandlung der Selbstreflexivität herbeiführen. Neben einer veränderten Alltagwahrnehmung und einem veränderten Selbstbewusstsein lässt sich in den Interviews eine starke Mensch-Technik-Verschränkung beobachten. So hat das Interface einen signifikanten Einfluss auf die Wahrnehmung, das Selbstempfinden und Handeln der Untersuchten. Diese äußert sich durch positive Gefühle, die allein das Aufblinken des Smartphones für die Nutzer*innen mit sich bringt, aber auch durch eine starke emotionale Bindung an das eigene Profil und die Kuration der Daten, die darin gesammelt werden (vgl. ebd., 5). Auch wenn die Pflege des Profils mit einigem Aufwand verbunden ist, ist Instagram attraktiv für die Nutzenden, da es eine Quelle der Anerkennung bildet. Likes werden in den Interviews als „Futter fürs Belohnungssystem“ (ebd., 6) bezeichnet, wodurch Likes und Kommentare zu außeralltäglichen Ritualen der Bestätigung werden, die potenziell zu jeder Zeit, nämlich nach dem Posten eines Bildes, verfügbar sind.

Dabei kann allein durch die Art der Datengenerierung keine Auskunft über alltägliche Vergleichs- und Bewertungspraktiken auf Instagram gegeben werden und die Frage, wie sich die Subjektivierungsweise auf der App konkret gestaltet, bleibt offen. Um diese Frage zu beantworten folgt die Darlegung der empirischen Ergebnisse im nächsten Kapitel. 


\subsection{Analyse I: Diskursive Interfaceanalyse}

Das leitende Interesse dieser Teiluntersuchung richtet sich nach der Frage, wie bestimmte Logiken und normative Annahmen in das Design und Interface von Instagram eingebettet sind. Welche normativen Vorstellungen werden an die Nutzenden herangetragen und welche Handlungsweisen werden nahegelegt?

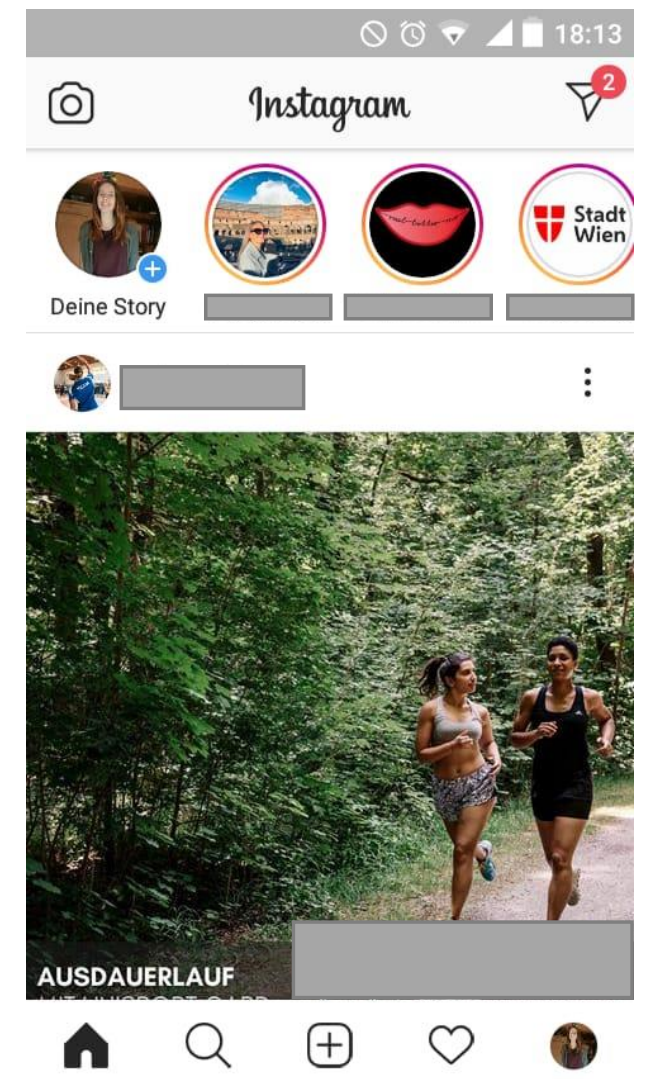

Abbildung 2: Startbildschirm Instagram (Screenshot erstellt am 18.09.2020)

Abbildung 2 zeigt den Startbildschirm, der beim Öffnen von Instagram angezeigt wird. Es lässt sich sehr gut der starke Fokus auf das Bild erkennen (vgl. Sheldon \& Bryant 2016, 92). Öffnet man die App, werden gut zwei Drittel in der Timeline durch den ersten Beitrag eingenommen. Im oberen Drittel werden die verschiedenen Stories angezeigt. Mit einem Klick auf einen dieser Kreise wird die Story abgerufen und nimmt dann den vollständigen Bildschirm ein. Zum Vergleich sind in Abbildung 3 zwei andere Plattform abgebildet, wobei Lokalisten (links) unter den ersten sozialen Netzwerken zählt und TikTok (rechts) eine der jüngsten erfolgreichen Plattformen ist. 

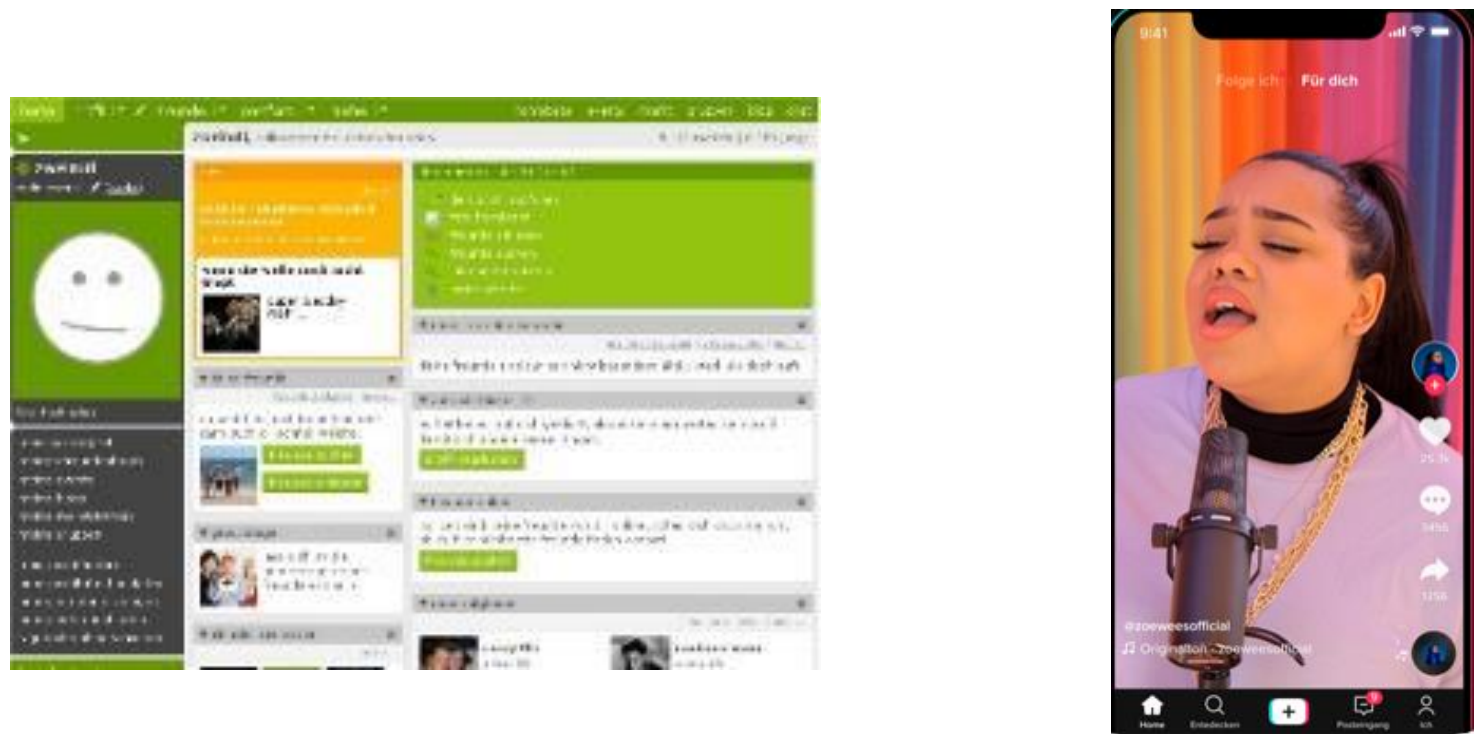

Abbildung 3: Lokalisten (links) und TikTok (rechts); Screenshot erstellt am 18.09.2020)

In der folgenden Analyse kristallisierten sich vier zentrale Aspekte heraus, die helfen, die Subjektivierungsform von User*innen auf Instagram besser zu verstehen. Diese sind (1) der Fokus auf das Bild, (2) die Konsumlogik, (3) Ästhetik und (4) die Spannung zwischen Intimität und Reichweite.

\section{Das Bild und der Körper}

Im Vergleich zu anderen Apps lässt sich eine gewisser „Siegeszug“ der Bilder gut erkennen. Während bei frühen Social-Networking-Sites noch der Text dominierte, rückt er immer weiter in den Hintergrund. ${ }^{31}$ Instagram stand bereits häufig in der Kritik für die realitätsferne Darstellung vor allem weiblicher Körper. Die Vermittlung ungesunder Schönheitsideale würde verstärkt zu Essstörungen bei Jugendlichen führen. Trends wie die DIN A4-Challenge ${ }^{32}$, die BellyButton-Challenge oder die Bikini-Bridge-Challenge ${ }^{33}$ wurden zwar nicht ausschließlich auf Instagram ausgetragen; dennoch hebt sich Instagram in dieser Debatte hervor, da dort vor allem Influencer*innen diese Ideale propagieren und deshalb in die Kritik geraten. Auch über andere

\footnotetext{
${ }^{31}$ Die Tendenz zu dieser Entwicklung, die bereits bei Instagram deutlich sichtbar wird, hat sich in den folgenden Jahren noch verstärkt. Bei Tiktok, der jüngsten global erfolgreichen Social-Media-App, erscheint in der mobilen Ansicht nur noch ein Beitrag über den vollständigen Bildschirm und kann durch Scrollen durch den nächsten ersetzt werden. Kommentare werden innerhalb des Videos angezeigt und haben keine eigene Leiste mehr (siehe Abbildung 3).

${ }^{32} \mathrm{https}: / / \mathrm{www} . w e l t . d e / v e r m i s c h t e s / a r t i c l e 153567432 /$ Wenn-die-Taille-so-schmal-wie-ein-A4-Blatt-seinmuss.html (zuletzt aufgerufen am 21.08.2020). Bei der DIN A4-Challenge posteten vor allem Frauen Bilder, auf denen sie sich ein DIN A4-Blatt vor den Bauch halten. Ziel der Challenge war es, dass die Taille so schmal ist, dass sie hinter dem Blatt nichtmehr zu sehen ist.

${ }^{33} \mathrm{https} / / / \mathrm{www}$.bento.de/style/paper-waist-challenge-neuer-schoenheits-trend-auf-instagram-a-00000000-0003-00 01-0000-000000439685 (zuletzt aufgerufen am 21.08.2020). Das Ziel der Belly-Button-Challenge ist es, die Hand über den Rücken zu führen und mit den Fingern den Bauchnabel zu berühren. Mit der Bikini-Bridge ist eine Lücke zwischen Bauch und Bikini-Hose gemeint, welche entsteht, wenn die Hüftknochen höher liegen als der Bauch und so das Bikini-Unterteil besagte „Brücke“ über den Bauch spannt.
} 
Social-Media- und Dating-Apps fand bereits eine ausführliche Debatte und Auseinandersetzung - auch im wissenschaftlichen Kontext - mit der starken Konzentration auf den Körper in Form von Bildern und Videos statt, in der kritisiert wurde, dass der Körper objektiviert werde. Vor allem auf Dating-Apps wie Tinder steht die Reduktion des Menschen auf sein virtuelles Abbild in der Kritik (Illouz 2012; Krüger \& Spilde 2019). Dadurch, dass das Bild als Platzhalter für menschliche Präsenz fungiert, reiht es sich in einen „Markt“ aus ähnlichen Bildern ein wodurch sich das Verhältnis zum eigenen und zu fremden Körpern verändert. „,[T]heir bodies being on display all the time - and which they have to compete with others on the basis of their bodies and in which the body becomes the main source of social and economic value" (ebd., $6)$.

Der Fokus auf das Bild (und zwar auf jeweils nur ein Bild pro Bildschirm) und den eigenen Körper wird vor allem deutlich, wenn man die Möglichkeiten untersucht, die das Interface bietet, um einen neuen Beitrag zu erstellen. ${ }^{34}$ In der Startansicht der App bieten sich dem oder der Nutzer*in nicht nur eine, sondern vier Möglichkeiten, ein neues Bild zu erstellen. Ganz oben links kann durch das Berühren des Kamera-Icons ( ( 0 ) oder durch Anklicken des eigenen StorySymbols die Frontkamera aktiviert werden. Innerhalb dieser Ansicht kann die Kameraperspektive gewechselt, oder ein bereits existierendes Bild oder Video aus der eigenen Galerie ausgewählt werden, wobei diese Funktion eher in den Hintergrund gerückt ist. Beide Aktionen enden in einem Post in der Story. Davor hat der Nutzende aber verschiedene Optionen zur Verfügung: Öffnet sich die Frontkamera, kann zum einen ausgewählt werden, ob ein Foto, ein Video oder ein Boomerang-Video erstellt werden möchte. Außerdem bietet sich die Möglichkeit, via Gesichtserkennung verschiedene Filter in Echtzeit auf das Gesicht projizieren zu lassen. Das heißt, es muss nicht erst ein Foto erstellt werden, das dann im Anschluss bearbeitet werden kann, da schon bei der Erstellung der oder die Nutzende sein durch den Filter verändertes Gesicht sieht. Auf der Startansicht ganz unten in der Mitte bietet ein Klick auf das Plus ( $\oplus$ ) die dritte Möglichkeit, einen neuen Beitrag zu erstellen. Eine zusätzliche vierte Möglichkeit, die Frontkamera zu öffnen, kann ein Swipe nach rechts bilden, der ebenfalls die Frontkamera aktiviert.

\footnotetext{
34 „Dank der visuellen Natur von Instagram können Menschen auf dieser Plattform wie auf keiner anderen ihren Leidenschaften nachgehen und diese teilen. Dies trifft insbesondere auf die Beauty-Community zu. [...] Das Geheimnis liegt darin, wie bei einem Komplettlook auch auf die Details und die Harmonie zwischen den einzelnen Elementen zu achten. So hat es Instagram geschafft, zur Beauty-Plattform par excellence zu werden. Einige der besten Inhalte auf Instagram gehören in den Bereich „Beauty“ und wurden entweder von Marken veröffentlicht, die ihre neuesten Produkte präsentieren, oder von Experten, die ihre Tipps und Tricks mit der Community teilen, oder aber von Verbrauchern, die ihrer Leidenschaft frönen“ (https://business.instagram.com/blog/beauty-on-instagram/; zuletzt aufgerufen am 21.08.2020).
} 
Das Posten eines Beitrages oder einer Story wird durch viele verschiedene Funktionen im Interface nahegelegt. Zum einen dominiert diese Funktion bereits durch die drei direkt auf dem Startbildschirm verfügbaren Icons deutlich vor anderen Anwendungsmöglichkeiten wie den Direct Messages $(\nabla)$ oder der Suchleiste $(Q)$. Zum anderen wird der Abbruch beim Erstellen einer Story oder eines Posts entsprechend sprachlich gerahmt. Erstellt man ein Bild und betätigt im Anschluss den „Zurück“-Button erscheint ein Pop-up-Fenster, in dem steht „Foto verwerfen? Wenn du jetzt zurückgehst, verlierst du dein Foto“, darunter in roter Schrift „Verwerfen“ und in schwarz „Behalten“. Dieser Text konstruiert eine persönliche Beziehung zwischen dem Bild und dem Nutzenden und auch das Wort „,verlieren“ agiert auf einer emotionalen Ebene. Etwas zu verlieren ist in den meisten Fällen negativ konnotiert - „,behalten“ klingt meist vorteilhafter.

Entscheidet man sich also, ein Bild oder Video in der App aufzunehmen, können nach der Erstellung noch weitere Filter angewendet werden. Zudem stehen verschiedene Gestaltungsoptionen zur Verfügung. ${ }^{35}$ Neben einem Stift und der Möglichkeit, Text hinzuzufügen, stehen eine Reihe weiterer Features offen. Die Ergänzung eines Standorts, die Verlinkung einer Person oder Hashtags reihen sich ein zwischen eine Spende, die integriert werden kann, Musik, die Uhrzeit, eine Umfrage, GIFs, verschiedene Sticker und Emojis und einem Regler, der von den Empfängern nach links geschoben werden kann (auch Emoji Slider genannt). Das Foto scheint hier anhand der Reihe an Zusatzfunktionen nicht für sich allein zu stehen, sondern legt durch viele Optionen nahe, dass eine nachträgliche Editierung wünschenswert ist.

\section{Konsumlogik}

Verbunden mit dem Fokus auf bildliche Darstellungen und Videos ist eine gewisse Konsumlogik tief in das Interface von Instagram eingebettet. Im Entdeckerfeed hat der oder die Nutzer*in die scheinbar endlose Auswahl an für ihn oder sie „relevanten“ Beiträgen. Auch der Feed der abonnierten Profile nimmt kein Ende und so kann man ewige Zeit durch die verschiedenen Beiträge scrollen. In den Stories folgt ein Beitrag nahtlos an den anderen. Fotos werden nach vier Sekunden durch das nächste abgelöst und ohne selbst aktiv zu werden, werden also alle Beiträge aus den Stories dem oder der Betrachter*in abgespielt. Die oberflächliche Betrachtung und der passive Konsum der geposteten Inhalte wird aktiv durch das Design und die schnelle

\footnotetext{
${ }^{35}$ Auf der offiziellen Website werden diese Optionen auch für Werbetreibende attraktiv dargestellt: „Instagram verfügt über eine breite Palette an Tools und Oberflächen, die Werbetreibende nutzen können, um für die Community Stories mit dem perfekten „Look“ zu erstellen. Mit den auf diese Weise entstandenen attraktiven Bildern und Videos möchte Instagram ,die Welt enger zusammenbringen“ (https://business.instagram.com/blog/beautyon-instagram/; zuletzt aufgerufen am 21.08.2020).
} 
Bildabfolge gefördert und macht damit eine tiefe Auseinandersetzung mit dem Geposteten unwahrscheinlicher. Wie ein Katalog kann man zwischen verschiedenen Lebensinhalten bzw. Lifelogs der Menschen hin und her swipen, ohne zwangsläufig in den wechselseitigen Austausch zu treten. Da die Stories sich nach 24 Stunden selbst löschen und auch in der Timeline oder im Feed selten ein Beitrag zweimal erscheint, verstärkt sich dieser Effekt.

Der Algorithmus der App sorgt dafür, dass der oder dem Nutzenden nur Beträge anzeigt werden, die sich in das eigene Relevanzsystem einfügen, das Instagram ausgehend von bisherigem Nutzungsverhalten und aus persönlichen Daten generiert. Diese Funktion wird innerhalb der App sprachlich sichtbar gemacht durch Hinweise wie „Videos, die dir gefallen könnten“ oder „mehr Beiträge wie diesen anzeigen“. Sucht man zum Beispiel nach Beiträgen zu Mode, bekommt man zukünftig mehr Fotos von Kleidung und Schmuck zu sehen, klickt man auf Beiträge mit Tanzvideos, erscheinen zukünftig mehr Tänzer*innen im Feed. Es liegt im Interesse der App, dass Menschen möglichst viel Zeit auf ihr verbringen; kurzweiliges Entertainment und endlose Unterhaltungsangebote tragen dazu bei.

Ein weiteres Indiz, wie die App den passiven Konsum von Inhalten und das oberflächliche Betrachten in ihrem Design präferiert, ist die Verankerung von Funktionen, die den direkten Austausch mit anderen Profilen ermöglichen. Diese tritt zugunsten der geposteten Inhalte relativ in den Hintergrund. Durch ein kleines Symbol in Form eines Papierfliegers kann die DirectMessage-Funktion aufgerufen werden. Diese ist im Grunde ähnlich aufgebaut wie auf vergleichbaren Apps. Zunächst erscheint eine Liste der letzten Chats, die dann einzeln aufgerufen werden können. Auch dort bildet der Text im Design nur noch eine untergeordnete Rolle. Farblich hervorgehoben sieht man unten im Eingabefeld ein Kamerasymbol, dann ein Freitextfeld,

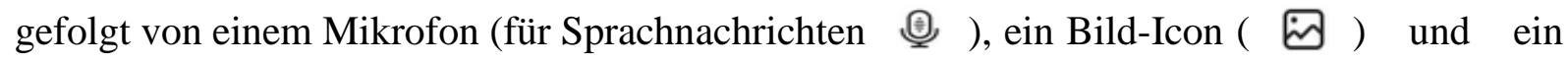
Icon für das Hinzufügen von Gifs oder Stickern ( $\odot$ ).

Um die Logik des Swipens besser zu verstehen, ist es wichtig, die dahinterstehende Wertschöpfungskette zu betrachten. Instagram profitiert direkt und indirekt davon, wenn Menschen sich möglichst lange auf der Plattform aufhalten und möglichst viel Content produzieren. Sowohl Nutzungsdauer als auch -häufigkeit und allgemeine Verbreitung entscheiden über die Preise von Werbeanzeigen und gesponserten Posts. Durch die Möglichkeit, endlos durch den Feed zu scrollen, wird ähnlich wie auf Tinder ein object of desire (ebd., 14) erzeugt, da potenziell hinter dem nächsten Wischen der perfekte Partner, oder im Falle Instagrams, ein spannender Beitrag 
lauert. Entscheidend ist dabei, dass sich dieses Objekt der Begierde von einem Objekt der Realität unterscheidet, sich so die Suche nach dem denkbar Begehrlichen perpetuiert und man immer weiter durch die Bilder und Videos wischt ohne an ein Ende zu gelangen.

Passend dazu gibt die große Auswahl an Editierungsmöglichkeiten in der Story dem User typische Instrumente des Marketings an die Hand. Leicht können blinkende Symbole, Strahlen, sich bewegende Ausrufezeichen oder auch Musik dem eigenen Bild oder Video hinzugefügt werden. Dadurch wird dem User nahegelegt, sich in einen Markt der Aufmerksamkeit einzufügen, in dem es gilt, aus der endlosen Masse an Posts herauszustechen und selbst Sichtbarkeit zu generieren.

\section{Ästhetik}

Der Ästhetik kommt auf Instagram ein großer Stellenwert zu und sie beginnt schon im Logo, das typografisch weich gestaltet ist (Abbildung 4). Die Farben des Logos sind ein weicher Farbverlauf, der sich auch innerhalb der App wiederspiegelt.

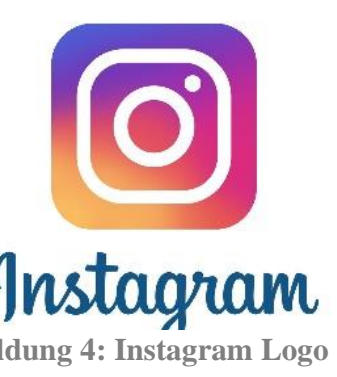

So ist zum Beispiel der Kreis, der eine Story anzeigt, in denselben Farben gehalten. Ansonsten ist das Design in der Grundeinstellung weiß. Auch der Slogan „Näher an den Menschen und Dingen die du liebst ${ }^{\star 36}$ passt zu einem sehr romantisch angehauchten Image. Das Herzsymbol ( $\bigcirc$ ) hat mehrere Funktionen und zugeschriebene Plätze auf Instagram. Zum Beispiel ersetzt das Herz den klassischen „Thumbs up“-Like wie er beispielsweise auf Facebook verwendet wird. Ein Bild kann entweder durch das Herzsymbol unterhalb des Beitrages oder durch Doppelklick auf das Bild geliked werden. Im zweiten Fall ploppt ein großes rotes Herz über dem Beitrag auf. Ein Herz wird gegenüber einem Daumen mehr mit Liebe, Wertschätzung und Emotionalität assoziiert, die rote Füllung des Symbols ist dabei emotional aufgeladener als das kühle Blau des Facebook-Icons. ${ }^{37}$ Auch DMs können durch einen Doppelklick mit einem Herz versehen werden, das sprachlich durch ein ,[Username] gefällt deine Nachricht“ dem Empfänger

\footnotetext{
${ }^{36} \mathrm{https}$ ://about.instagram.com/ (zuletzt aufgerufen am 21.08.2020).

${ }^{37} \mathrm{Zu}$ Farben im Webdesign siehe (Bartel 2014).
} 
angekündigt wird. Eine weitere Verwendung findet das Herz-Icon unten in der Leiste des Startbildschirms, das zu den Aktivitäten und Benachrichtigungen führt. In diesem Untermenü sind Abo-Anfragen und neue Likes in chronologischer Reihenfolge aufgelistet. Auch in den sogenannten „Quick Reactions“ (Abbildung 5) zieht sich Wertschätzung und ein positives „Feel Good“-Gefühl weiter durch das Design. Innerhalb der Story gibt es außerdem

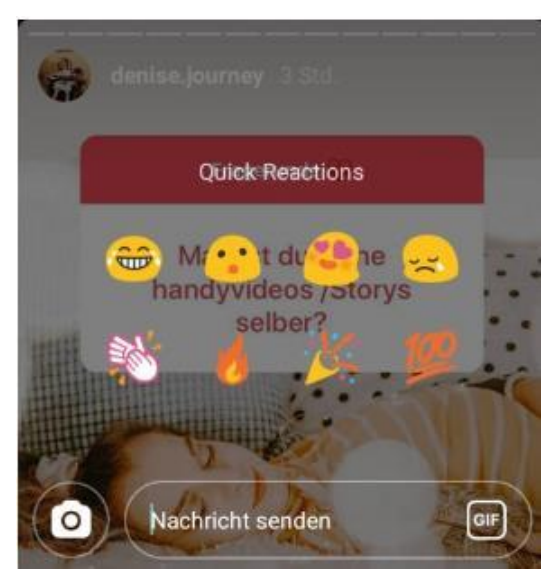

Abbildung 5: Screenshot Quickreactions (2020-06-28-11-47-49)

die Möglichkeit, mit einem Schieberegler die Reaktion des Betrachters einzufordern. Voreingestellt ist dort ein Emoji mit Herzaugen. Die Möglichkeiten, Feedback auf Inhalte jeglicher Art zu geben, ist also durchgängig positiv konnotiert und entscheidet man sich für eine dieser Möglichkeiten, kann dies nur in Form von Wertschätzung und Anerkennung erfolgen. Negatives Feedback oder Kritik wird durch keine Menüoption angeboten, ist aber durch die Freitexteingabe jederzeit möglich.

Die romantisierenden und nostalgischen Eigenschaften des Interface werden auch durch die Gestaltung der verschiedenen Icons deutlich. Das Logo ähnelt einer Polaroid-Kamera, Herzen werden, wie bereits erläutert, mehrfach aufgegriffen und das DM-Icon ist ein Papierflieger. All diese Icons haben einen affektiven, emotionalen und romantischen oder nostalgischen Charakter und konstruieren so Instagram als wertschätzenden, intimen, zwischenmenschlichen Raum. Der wertschätzende, positive Interaktionsrahmen, der durch das Interface nahegelegt wird, wird auch später in der zweiten Analyse relevant werden. 


\section{Intimität und Reichweite}

Betrachtet man die Art und Weise, wie soziale Beziehungen auf Instagram gerahmt werden, scheint sich ein Spannungsverhältnis zwischen Intimität und Reichweite zu offenbaren, das besonders interessant zu betrachten ist. Wie bereits skizziert, ist das Interface darauf ausgelegt, eine warme, intime Atmosphäre zu konstruieren, die auch durch den Aspekt der Spontanität unterstrichen wird. Komparative Studien, die sich bereits vor fünf Jahren mit den Unterschieden zwischen Facebook und Snapchat beschäftigt haben, haben gezeigt, dass sich selbst löschende Nachrichten ein höheres Gefühl von Intimität erzeugen als permanente Posts und Nachrichten. Nachrichten auf Snapchat zeichnen sich durch ihre autolytische Natur aus, da sie sich löschen, nachdem sie vom Empfänger oder der Empfängerin betrachtet wurden (vgl. Vaterlaus et al. 2016). Stories auf Instagram funktionieren nach einem ähnlichen Prinzip, da sie nach 24 Stunden verschwinden und so sie selbst und sich möglicherweise aus ihnen ergebende Konsequenzen einen engen zeitlichen Rahmen nicht verlassen.

Öffnet man die Storyfunktion, erscheint die Frontkamera. Der oder die Nutzende soll also ein spontanes Bild oder Video erstellen und eher nicht ein zuvor aufgenommenes Bild auswählen. Damit folgt die App einem weiter zurückreichenden Trend der Fotografie, der weggeht von der Aufnahme besonderer Ereignisse und inszenierter Familienbilder hin zur Aufnahme flüchtiger Alltagsmomente. Diese werden oft genutzt, um eine Art der co-presence oder co-experience zwischen verschiedenen Menschen zu schaffen, die sich nicht face-to-face begegnen (vgl. Koskinen 2007). Fotos und Videos spielen also immer weniger eine Rolle zur langfristigen Archivierung, sondern werden eher im Sinne eines „Ich denke an dich“ erstellt und mit anderen geteilt. Dieser Gedanke wird auch durch den Slogan getragen, der eine nahe Beziehung mit geliebten Menschen verspricht. Durch die Möglichkeit, neben Textnachrichten auch über Ton-, Bild-, Videoaufnahmen oder grafische Elemente wie Gifs zu kommunizieren, werden die Interaktionen der klassischen ,face-to-face“-Interaktion angenähert, da mehr Modalitäten zur Auswahl stehen. Dieser Aspekt der Spontanität wird auch in den privaten Nachrichten aufgegriffen, die automatisch mit „gelesen“ markiert werden. Die Markierung einer versendeten Nachricht als ,gelesen“ erhöht den sozialen Druck zu einer sofortigen Antwort auf den Empfänger und provoziert ein zeitnahes und schnelles Antwortverhalten. ${ }^{38}$

\footnotetext{
${ }^{38}$ Das dadurch vermutlich entstehende schnellere Antwortverhalten kann ebenfalls dazu dienen größere Intimität zu suggerieren, da ein solcher Chat sich einem face-to-face Gespräch eher annähert als bspw. ein Emailwechsel mit selteneren Antworten. Gleichzeitig werden sich User*innen dadurch länger auf der Plattform aufhalten, was wiederum den ökonomischen Wert Instagrams steigert.
} 
In starkem Kontrast dazu steht die Anzeige des Profils und verschiedener Optionen, die darauf ausgelegt sind, eine hohe Reichweite und Sichtbarkeit über die eigene Peer-Group hinaus zu erzeugen. Dabei müssen aber grundsätzlich zwei Profiloptionen unterschieden werden. Jedem und jeder Nutzer*in steht die Option frei, das eigene Profil öffentlich oder privat einzustellen. Ist das eigene Profil privat, muss ähnlich wie auf Facebook eine Abo-Anfrage versendet und bestätigt werden, um Inhalte zu sehen. Bei einem öffentlichen Profil ist der Zugang wesentlich niederschwelliger und es kann durch einen einfachen Klick auf „Folgen“ abonniert werden oder über die Suchfunktion das Profil inklusive Stories und Posts aufgerufen werden. Die Buttons „Folgen“ oder „Abonnieren“ sind beinahe die einzigen, die auf Instagram farblich hervorgehoben werden. Ihnen kommt offensichtlich ein besonderer Stellenwert zu, da sie sich durch diese Gestaltung optisch klar von anderen Optionen abheben. Betrachtet man die Profilseite, ist interessant, dass dort nur ein sehr kleiner Bereich für das Profilbild reserviert ist und der größere Fokus auf drei quantitative Aspekte gelegt wird: Die Anzahl der geposteten Beiträge, die Anzahl der Abonnent*innen und die Anzahl der abonnierten Personen. Damit findet in der Gestaltung der Profilansicht ein Bruch mit dem Fokus auf das Visuelle statt, der den Wettbewerbscharakter verdeutlicht, der auf Instagram durch die Ästhetik zwar verschleiert wird, dem hier aber eine zentrale Rolle zukommt. In der Profilansicht wird der oder die User*in auf drei Kennzahlen reduziert, die den vermeintlichen Wert bzw. die Popularität und Aktivität auf Instagram in den Vordergrund rücken.

Alles deutet darauf hin, dass Vernetzung und Kommunikation neben dem Posten von Bildern und Videos wichtige Optionen auf Instagram sind. Auf eine hohe Vernetzung ausgelegt sind die Hashtags, die es erlauben, Content thematisch zu markieren. Durch die Verwendung populärer Hashtags kann die Aufmerksamkeit auf das eigene Profil gelenkt werden, was häufig dazu dient, eine hohe Followerzahl zu generieren. Spezifische Hashtags ermöglichen die Verknüpfung durch gemeinsame Interessen oder Ansichten. Interessiert man sich beispielsweise für Sport, können durch die Suche nach einem entsprechenden Hashtag relevante Inhalte und Profile gefunden werden. Ebenfalls über Hashthags funktionieren sogenannte Challenges, wie die bereits angesprochene DIN A4-Challenge. Darüber ist es möglich, über verschiedene soziale Gruppen hinweg mit anderen an verschiedenen Challenges teilzunehmen und damit Gemeinsamkeiten und gemeinsame Erfahrungen zu suggerieren. Neben der thematischen Vernetzung durch Hashtags können auch Personen getaggt, d.h. in einem Post markiert, bzw. verlinkt werden. Auch diese durch das Interface konstruierte Möglichkeit führt zu einer höheren Interaktivität, die zu Vergleichen und Bewertungen einlädt. Dabei übersetzt die Anzeige der Abonnier- 
ten und Abonnenten auf dem jeweiligen Profil diese Vernetzung in eine Art quantitative Währung und suggeriert die Vorteilhaftigkeit eines möglichst großen Netzwerks. Diese Übersetzung des heterogenen Netzwerks in eine einfache Zahl existiert bereits auf anderen Social-MediaSeiten, erhält aber auf Instagram eine neue Qualität, wenn der Wert von Influencer*innen vor allem in ihrer Rolle als Werbeträger*innen anhand ihrer Followerzahlen bemessen wird. Zusammengenommen zielt Instagram darauf ab, dass Menschen private, spontane und intime Inhalte ihres Lebens mit einer möglichst großen Gruppe signifikanter Anderer teilen und sich gemeinsam öffentlichen Vergleichen und Bewertungen in Form von Likes und Kommentaren unterziehen.

\section{Exkurs: Wie wird man zum/zur Influencer*in}

Während der Feldphase wurde die Frage nach der Bedeutung kommerziell genutzter Profile immer dringlicher. Die im nächsten Kapitel vorgestellten Daten beinhalten eine Frau mit ca. 20.000 Followern, die damit schon zu den sogenannten Mikroinfluencern zählt. Diese Mikroinfluencer befinden sich meist an der Schnittstelle zwischen privaten Profilen und denen großer Influencer*innen, deren Profil zum Hauptberuf geworden ist.

Instagram wirbt auf seiner offiziellen Homepage mit der Wirkmacht von Produktplatzierungen in Stories. So entwickeln laut Instagram 68 \% der Menschen eher Interesse für ein Unternehmen oder Produkt, wenn sie es in einer Story gesehen haben. ${ }^{39}$ Klares Argument dafür ist immer wieder die authentische und an den Alltag geknüpfte Darstellung des Produkts oder Unternehmens. In den Stories ist eine Differenzierung zwischen kommerziellem und nicht kommerziellem Content oft kaum noch möglich und die Grenzen verschwimmen zusehends. Anders als in klassischen Medien merkt der Betrachter oder die Betrachterin oft keinen Unterschied mehr zwischen Werbung und restlichem Inhalt.

Influencer*innen nehmen in diesem Marketingsystem die Rolle der menschlichen Litfaßsäule ein - sie sind die Werbeträger*innen, die Produkte in ihren Alltag integrieren, wodurch die Inszenierung ihres Lebens zum Vollzeitjob wird. Um diese Prozesse genauer zu verstehen und in den Daten sensibel zu sein für digitale Selbstdarstellung und strategische Selbstinszenierung, wurden Online-Videos und Anleitungen zum Leben als Influencer*in gesichtet und an einigen Stellen ergänzend in der Ergebnisdarstellung angeführt. In diesen Videos zeigen oft Menschen, die selbst eine hohe Reichweite auf Instagram haben, wie sie eine hohe Followerzahl erreicht und ihr Hobby zum Beruf gemacht haben. Diese Videos sind also zwischen den Subjekten und

\footnotetext{
${ }^{39} \mathrm{https}$ ///about.instagram.com/features/stories (zuletzt aufgerufen am 15.06.2020).
} 
dem Diskurs zu verorten und zeigen Strategisierungstechniken, die genutzt werden, um gezielt mehrere Tausend Follower anzusprechen. Dabei erreichen diese Ratgeber bis zu 800.000 Aufrufe auf YouTube, was ihre Relevanz verdeutlicht. ${ }^{40}$

Alle Anleitungen, Dokumentationen und Erfolgsgeschichten einen drei „Erfolgskriterien“: regelmäßigen ,guten“ Content, der die Person und ihr Leben in den Fokus rückt, Authentizität und die intensive Community-Pflege. Mehrmals am Tag im Feed und in der Story zu posten, wird innerhalb dieser Ratgeber als ebenso wichtig eingestuft wie durch die Festlegung auf einen Filter für Einheitlichkeit im eigenen Feed zu sorgen. Darüber hinaus sind Einzigartigkeit und Ästhetik zentrale Schlagbegriffe für eine strategische Nutzung Instagrams.

Zusammengefasst zeigt die Analyse des Interface, dass die Handlungsmöglichkeiten, die Instagram nahelegt, darauf ausgelegt sind, möglichst viele Daten in Form von Posts in Stories und im Feed zu erzeugen. Die starke Hervorhebung der Frontkamera im Design offenbart eine Konzentration auf Bilder im Allgemeinen und Körper im Besonderen, die auf der Plattform geteilt werden sollen. Die Übersetzung des Lebens in Daten soll also vor allem in Form qualitativer Inhalte erfolgen. Zahlen spielen „nur“ in Form von Likes und Followern eine Rolle. Damit verbunden ist die Fokussierung auf Bildliches mit verschiedenen Möglichkeiten in Interaktionen mit anderen User*innen zu treten, wobei eine öffentliche und für alle Follower sichtbare Kommunikation in Form von Kommentaren oder Tags vor privaten Direktnachrichten präferiert wird. Dadurch evoziert das Interface öffentlich sichtbare Vergleiche und Bewertungen über Bilder, auf denen vor allem die User*innen selbst zu sehen sind. Dabei werden zum einen ästhetische Werte durch das Interface vermittelt, aber auch Konsum- und Konkurrenzlogiken sind wichtiger Bestandteil Instagrams.

\footnotetext{
${ }^{40} \mathrm{https}: / / \mathrm{www} . y o u t u b e . c o m / r e s u l t s ? s e a r c h \_q u e r y=w i e+w e r d e+i c h+i n f l u e n c e r(z u l e t z t$ aufgerufen am 16.09.2020).
} 


\subsection{Analyse II: Online-Ethnografie}

Die erste Teiluntersuchung konnte zeigen, welche Anforderungen an die Subjekte auf Instagram herangetragen werden und welche Vorstellung einer ,richtigen“ Nutzung in das Interface eingebettet ist. Da die Arbeit das Ziel verfolgt, nicht nur die Subjektformierung, sondern auch die Subjektivierungsweise auf Instagram zu beleuchten, schließt sich in diesem Kapitel die zweite Teiluntersuchung an. Es steht die Frage im Zentrum, wie User*innen Instagram nutzen und welche Handlungsmuster sich beobachten lassen, wobei ein besonderes Augenmerk auf Vergleich- und Bewertungspraktiken mit, durch und über qualitative Inhalte (z.B. Bilder) gelegt wird.

\subsubsection{Datengrundlage}

Die Datengrundlage des digital ethnografischen Teils bilden fünf Frauen, die im Folgenden kurz vorgestellt werden. In Abbildung 6-Abbildung 9 sieht man die verschiedenen Profilansichten. Darauf ist neben der Anzahl der bereits geposteten Beiträge die Anzahl der Follower und die Anzahl der abonnierten Profile zu sehen. Darunter befindet sich jeweils eine kurze Profilbeschreibung, die sogenannte Bio. Eine schlaglichtartige Beschreibung der Profile soll helfen, einen ersten Eindruck über die Daten zu gewinnen.

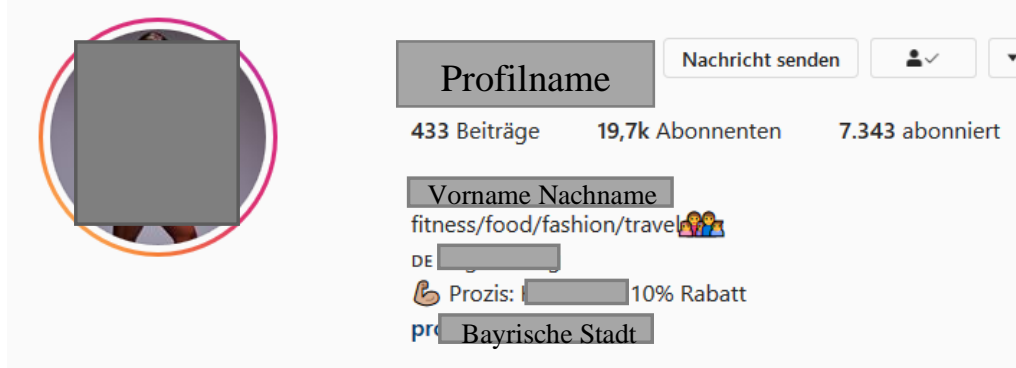

Abbildung 6: Profil Kathi

Kathi $^{41}$ kann als Mikroinfluencerin ${ }^{42}$ bezeichnet werden, da sie über 10.000 Follower besitzt. Sie macht ihr Hobby zunehmend zum Beruf, indem sie täglich über ihren Alltag als Mutter und Fitnessmodel berichtet. Ihrem Profil kann man ihren vollständigen Namen und ihren Wohnort entnehmen, mit ihren Interessengebieten „fitness, food, fashion, travel“ repräsentiert sie für Instagram typische Themen. Ihre Familie wird durch ein Emoji im Profil widergegeben. Prozis

\footnotetext{
${ }^{41}$ Die Profilnamen wurden anonymisiert und auch der Nachname wurde geschwärzt, falls er in der Bio genannt wurde. In der folgenden Analyse werden die Frauen immer bei ihrem Vornamen genannt, wobei die Namen geändert wurden. Da keine der Untersuchten eine Anonymisierung der Bilder gewünscht hat, werden diese unverfälscht abgebildet.

${ }^{42}$ Als Mikroinfluencer werden User*innen bezeichnet, die zwar weniger Reichweite besitzen als berühme Influencer, deren Profil meist auch ihr Beruf ist, aber trotzdem eine höhere Reichweite als durchschnittliche User*innen haben. Meist wird ab 10.000 Followern von Mikroinfluencern gesprochen.
} 
ist eine Marke, deren Angebot von Proteinriegeln bis zu Schlafkapseln reicht und deren Produkte Kathi regelmäßig in ihren Stories bewirbt. Ihr Feed ist geprägt durch Selfies oder Bilder aus Fotoshootings, oft ist dabei ihr Sixpack zu sehen.

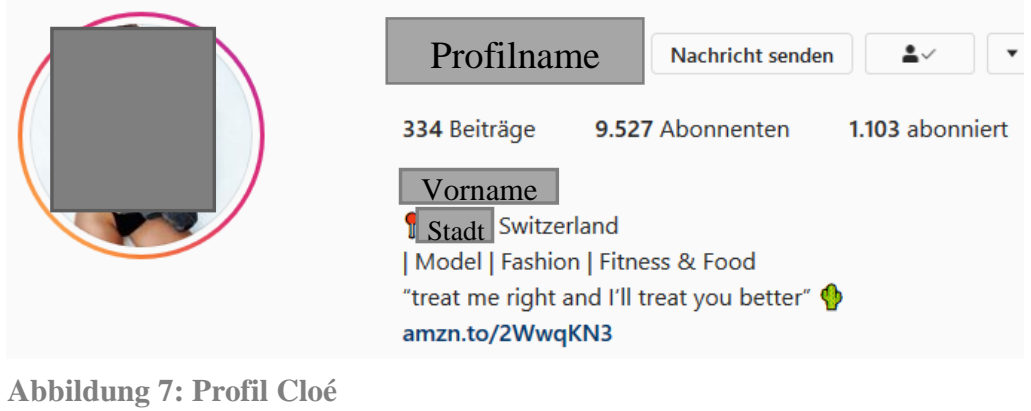

Cloé wurde über eine persönliche Freundin in die Forschung aufgenommen. Cloé lebt in Zürich und ergänzt ihre Profilbeschreibung durch „Model, Fashion, Fitness und Food“ und ein englisches Zitat, das mit einem Kaktus-Emoji abgeschlossen wird. Der Amazon-Link führt zur Produktseite einer elektrischen Gesichtsreinigungsbürste. In ihrer Story zeigt sie ihre täglichen Outfits, dokumentiert Restaurantbesuche mit Freunden und Eindrücke aus ihrer Stadt oder dem Urlaub. In ihrem Feed dominieren professionell aussehende Bilder von ihr, aber auch ein Tiger und Strandaussichten sind abgebildet. Mit 9.500 Followern ist sie dabei zur Gruppe der Mikroinfluencerinnen zu zählen, Produktplatzierungen sind allerdings selten.

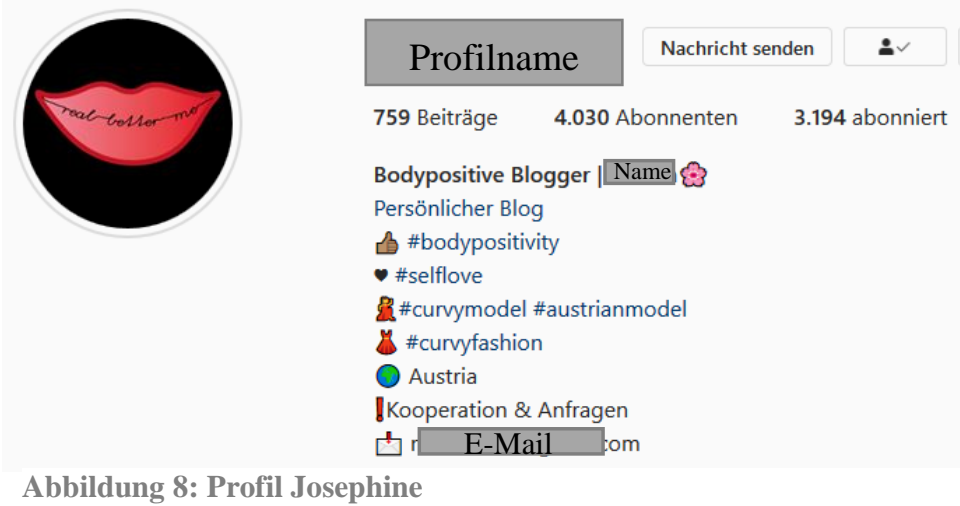

Josephine passt nicht in das klassische Schönheitsideal, dem Kathi und Cloé entsprechen. Sie beschreibt sich selbst als Bodypositive-Bloggerin, lebt in Österreich und ihre Profilbeschreibung enthält die Hashtags \#bodypositivity, \#curvymodel, \#austrianmodel, und \#curvyfashion. Sie benutzt keine Filter in ihren Stories, in denen sie vor allem Einblicke über ihren Alltag gibt, wie sie sich an diesem Tag fühlt, Ausflüge in die Natur, aber auch oft Ausschnitte aus Büchern oder Posts aus anderen Profilen, die sich mit Selbstliebe und Bodypositivity beschäftigen. In ihrem Feed postet sie Selfies und Bilder, die ihren Körper unbearbeitet zeigen, aber auch Bilder aus Shootings sind enthalten. 


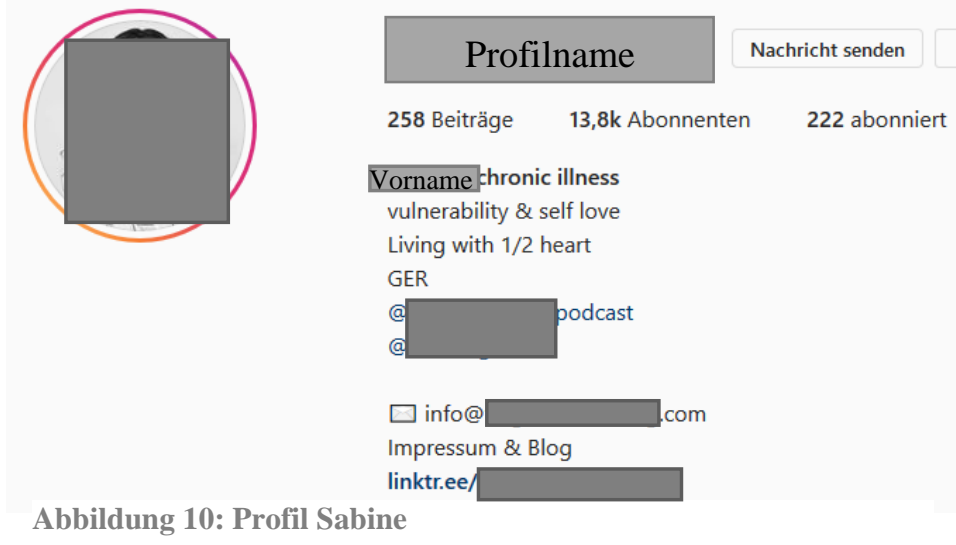

Sabine ergänzt ihren Namen durch „chronic illness“, darunter ist „,vulnerability \& self love“ und ,living with $1 / 2$ heart“ zu lesen. Außerdem verweist sie auf zwei Profile und ihren Blog, auf dem sie über ihr Leben mit einer chronischen Krankheit berichtet. Dieses Thema findet sich auch oft in ihrer Story wieder, wo sie der Betrachter oder die Betrachterin zu Arztbesuchen oder vor Krankenhausaufenthalten virtuell begleitet. Sie mag es Sonnenauf- oder -untergänge zu posten und teilt darüber hinaus oft Beiträge anderer Profile, die sich gegenseitig unterstützen oder sich auch mit chronischer Krankheit auseinandersetzen. Ihr Feed ist sehr ästhetisch aufgebaut und enthält neben Bildern von ihr auch Bilder von Blumen, Kaffeetassen oder mit Scrabble-Buchstaben gelegten Zitaten oder Wörtern.

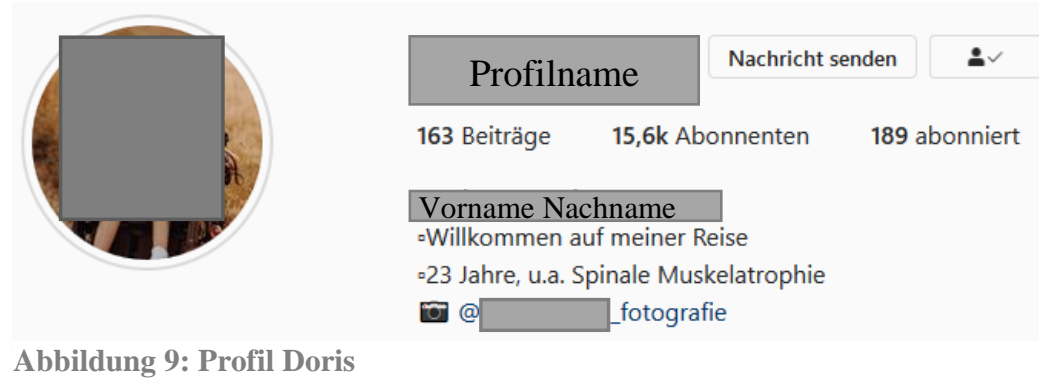

Doris heißt den Besucher oder die Besucherin auf ihrem Profil willkommen auf ihrer Reise, wobei sie nicht spezifiziert, wohin die Reise geht. Sie ist 23 Jahre alt und lebt mit Spinaler Muskelatrophie. Ebenso wie Sabine zählt sie ebenfalls über 10.000 Follower. In ihrer Story berichtet sie über ihren Alltag und ihre Ausbildung als Hundetrainerin. Sowohl in ihrer Story als auch in ihrem Feed klärt sie über Vorurteile über Menschen mit Behinderung auf und erreicht damit teilweise bis zu vier Millionen Menschen. ${ }^{43}$ Außerdem fotografiert sie gerne und verweist in ihrer Profilbeschreibung auf ein Fotografie-Profil, das sie zusammen mit einer Freundin führt.

\footnotetext{
${ }^{43}$ Da ihr Profil öffentlich ist, können auch Menschen, die ihr nicht folgen, ihre Videos zum Beispiel im EntdeckerFeed sehen. Das erklärt wieso einzelne Videos bis zu 4 Millionen Aufrufe zählen, obwohl sie „nur“ rund 15.000 Follower hat.
} 
Insgesamt macht die Vorstellung der Profile deutlich, dass die Frauen jeweils sehr unterschiedliche Interessen haben und auch die Zwecke der Profile unterscheiden sich. Während Doris und Sabine, aber auch Josephine vor allem über ihr Leben berichten, um andere Menschen aufzuklären und durch die digitale Darstellung ihres Alltags versuchen, sich im Bereich des „Normalen“" zu verorten, steht bei Cloé und Kathi vor allem die Darstellung ihres Körpers und ihres Lifestyles im Mittelpunkt. So werden je nach Profil unterschiedliche Schwerpunkte gesetzt und damit verbunden auch unterschiedlicher Content produziert.

\subsubsection{Darstellung der Ergebnisse}

Die Ergebnisdarstellung wird im Folgenden thematisch gegliedert, um unterschiedliche Praktiken auch mit Blick auf die Forschungsfrage kondensiert herauszuarbeiten. Dieses Kapitel soll zeigen, wie sich Lifelogging auf Instagram gestaltet und welche konkreten Handlungspraktiken sich beobachten lassen. In den Daten nimmt die möglichst ästhetische Inszenierung des eigenen Alltags und der eigenen Person einen sehr zentralen Stellenwert ein und bildet deshalb den ersten thematischen Abschnitt dieses Kapitels. Diese Tatsache spiegelt sich in der bereits existierenden Literatur, allerdings gelingt es aufgrund des ethnografischen Zugangs, detaillierte Einblicke in die Art und Weise dieser Inszenierung zu gewinnen. Überraschend war die hohe Community-Orientierung und die starke Reziprozität, die sich in den Stories und Kommentaren finden lässt. Die Selbstinszenierung steht nicht für sich, sondern ist immer eingebunden in die Interaktion und Kommunikation mit relevanten Anderen. Damit verbunden sind Strategien, die eingesetzt werden, um auf der einen Seite die Reichweite zu vergrößern, auf der anderen Seite auch innerhalb der schon bestehenden Followergruppe Aufmerksamkeit zu generieren. Die marktförmige Struktur, in die sich Posts fast zwangsläufig einordnen, macht Aufmerksamkeit zu einer knappen Ressource, die es in unterschiedlichem Maße zu gewinnen gilt. Eingeflochten in die verschiedenen Unterpunkte sind Akte der Widerständigkeit, die sich an vielen Stellen beobachten lassen. Das Material zeigt, dass die Nutzerinnen sich innerhalb der Strukturen, die das Interface ihnen vorgibt, kreativ bewegen und diese auch dazu nutzen, um alternative Vorstellungen und Werte zu vermitteln. 


\section{(Öffentliche) Interaktionen mit der Community}

Allgemein spielt die Community in den Daten eine größere Rolle als vor der Untersuchung angenommen. In der existierenden Forschung steht die Selbstinszenierung und -darstellung oft als Motiv stark im Vordergrund. Bilder von sich ins Netz zu stellen, ist zwar auf Instagram sehr zentral, die geposteten Inhalte sind allerdings in ein starkes Geflecht sozialer Interaktionen eingebunden. Die Bilder und Videos, die auf der Plattform geteilt werden, werden nicht nur kommentiert und geliked, sondern auch außerhalb dieser beiden Reziprozitätsangebote verhandelt, bewertet und verglichen. Durch DMs, Reposts in den Stories oder Bildunterschriften werden vielseitig Kommunikationsangebote gemacht und es wird mit den Followern interagiert.

Die Daten zeigen also, dass auch unter den Mikroinfluencer*innen die Interaktion über das hinausgeht, was Alice Marwick als „Parasozialität“ bezeichnet (Marwick 2015). Das Interaktionsangebot, das den Followern unterbreitet wird, ist durchaus ernst gemeint und es wird viel Zeit darauf verwendet, jede Nachricht persönlich zu beantworten, auch wenn sich sehr viele Nachrichten im Postfach befinden. ${ }^{44}$ In allen untersuchten Profilen kommt der Community ein hoher Stellenwert zu und die Follower scheinen keineswegs als anonyme Masse wahrgenommen zu werden. Vielmehr werden die Follower als geliebte und wertgeschätzte Mitglieder einer Community gerahmt, ungeachtet der Tatsache, ob eine persönliche Offline-Beziehung besteht oder nicht. Damit bildet sie einen integralen Bestandteil der Interaktionsfläche und geht weiter über eine gedachte Bezugsgröße hinaus. Das Publikum bzw. die Öffentlichkeit spielt also eine wichtige Rolle auf Instagram, die in Form von Likes, Followern und Kommentaren, DMs, Antworten auf Umfragen usw. ständig (technisch vermittelt) präsent sind (vgl. Oswald 2019, 10). Als Folge der hohen Community-Orientierung arbeitet auch Sascha Oswald in den von ihm durchgeführten Interviews heraus, dass das eigene Handeln und die Motivation auf eine bestimmte Wirkung ausgerichtet sind und auch den Wünschen des Publikums angepasst werden. Diese Anpassung stellt das einen notwendigen Faktor dar, wenn Mikroinfluencer*innen sie sich die Aufmerksamkeit auf längere Zeit sichern wollen (vgl. ebd., 8).

Die Interaktion mit der Community kann dabei auf unterschiedliche Weise gestaltet werden. Kathi fragt oft beiläufig nach dem Wohlbefinden ,ihrer Lieben“ und beginnt prinzipiell den Großteil ihrer Stories oder auch Posts mit „Hallo meine Lieben“, um im Anschluss über ihre

\footnotetext{
${ }^{44}$ Eine bekannte deutsche Influencerin erzählt in einem Youtube-Video, dass sie täglich bis zu vier Stunden in die Beantwortung von privaten Nachrichten investiert (https://www.youtube.com/watch?v=-EG9RfUPr3M; zuletzt aufgerufen am 31.08.2020) und auch in den Daten finden sich Hinweise darauf, etwa wenn eine der Frauen sich bei ihren Followern entschuldigt, dass sich noch nicht auf alle Nachrichten geantwortet hat und es sich für die nächste Zeit vornimmt.
} 
Pläne für den Tag, ihr Work-Out-Programm oder die Zubereitung ihrer Mahlzeiten zu sprechen. ${ }^{45}$ Abbildung 11 zeigt eine solche beispielhafte beiläufige Ansprache an die Follower:

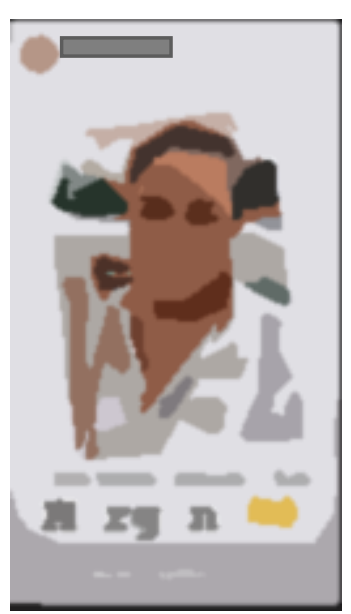

„Einen wunder-wunderschönen Guten Morgen, meine Lieben. I-ich genieße gerade mein zweites Frühstück (beißt von Riegel ab) Melty-Time (kaut) endlich hab ich sie wieder (kaut) ich hab sie so vermisst. Gottseidank gibt es echt ne Express-Lieferung bei Prozis (kaut) mhhh“

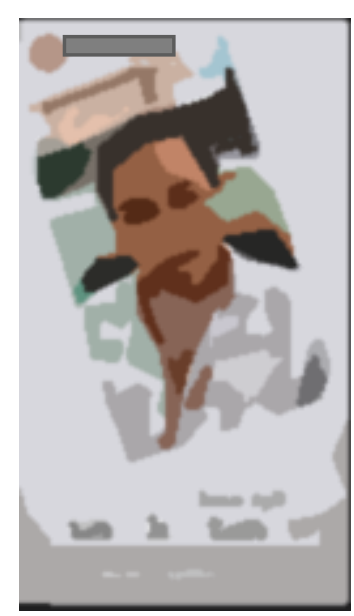

„Wie sieht euer Tag heute aus? Das Wetter ge-eht jetzt (schwenkt die Kamera kurz höher, sodass man den Garten sieht) gerade eigentlich (kaut) jetzt eigentlich ist grad ein bisschen Sonne (blickt zur Seite) hmm genau, bis jetzt ist noch Bad-HairDay (blick nach unten) $[\ldots]^{\text {“ }}$

Abbildung 11: Story Kathi (2020-06-05-20-18 Pos. 11-12)

In anderen Stories geht sie explizit auf Nachrichten ein, die ihr geschickt wurden: „Jetzt zeig ich euch mal bisschen was vom Garten, denn viele schreiben immer sie würden gerne den Pool mal ganz sehen und ja, wollen eventuell auch einen bauen. Also nehm ich euch jetzt mal ein bisschen mit (Wechsel auf Rückkamera)“(20200612_221139 Pos. 17). Im Anschluss daran geht sie mit dem Handy durch den Garten, erklärt die technischen Details des Pools und gibt Tipps für die Beschaffung. Einem ähnlichen Muster folgen Stories, in denen sie „ihren Lieben“ erklärt, wie sie ihr Workout durchführt oder was sie zum Frühstück isst. Dabei nimmt sie eine Art Vorbildfunktion ein, da sie Tipps und Ratschläge gibt, die dabei helfen, ihrem Lebensstil nachzueifern.

Auch in den anderen Profilen nehmen die Frauen häufig eine Vorbildrolle ein, zum Beispiel, wenn sie darüber sprechen, wie sie ihren Weg zu mehr Selbstliebe oder Akzeptanz ihres Körpers gemeistert haben. Durch diese Stories wird darüber hinaus deutlich, wie stark die Frauen im Fokus der zahlreichen Beobachter*innen stehen. Cloé spricht in einer ihrer Stories darüber, dass sie zahlreiche Anfragen erhält und sich ihre Follower fragen, wieso ihre Haare manchmal länger und manchmal kürzer aussehen. Indem sich die Frauen täglich filmen und diese Videos mit bis zu 20.000 Menschen teilen, bleibt kein Detail unbemerkt. Cloé erstellt aufgrund dieser Anfragen eine Reihe von Videos, in denen sie nach der Ankündigung: „wie mache ich das

\footnotetext{
${ }^{45}$ Mehr zur beiläufigen Ansprache an die Follower auf Instagram siehe Marwick 2015
} 
nur??““ (2020-07-07-16-37-28) über ihre Haare aufklärt. Sie spricht über verschiedene Haarverlängerungsoptionen, stellt ihre favorisierte Extensionsmarke vor und nimmt die Follower sogar mit an den Ort, an dem sie ihre Haarverlängerungen kauft.

Die Vorbildrolle ist dabei aber nicht nur ihnen vorbehalten. Manchmal kehrt sich die Relation um und die Follower nehmen eine Expertenrolle ein, indem sie nach Rat oder nach ihrer Meinung zu gewissen Themen gefragt werden. Ein sehr eindrückliches Beispiel lässt sich bei Josephine finden. Sie fühlt sich in einer gewissen Situation unwohl und bespricht ihre Gefühle bei einer Tasse Kaffee mit ihren Followern:

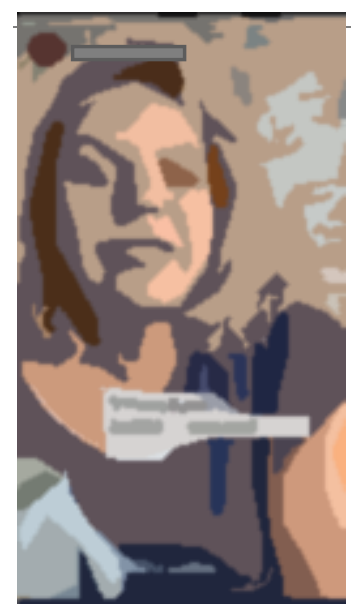

„So, ich wünsche euch einen schönen Nachmittag, eigentlich wollte ich heute noch was reden mit euch. Und zwar geht's um Sport. Wer von euch ist denn sportlich und was machts ihr so. Bei mir ist nämlich so, ich war früher nicht wirklich unsportlich $[\ldots]^{\text {“ }}$

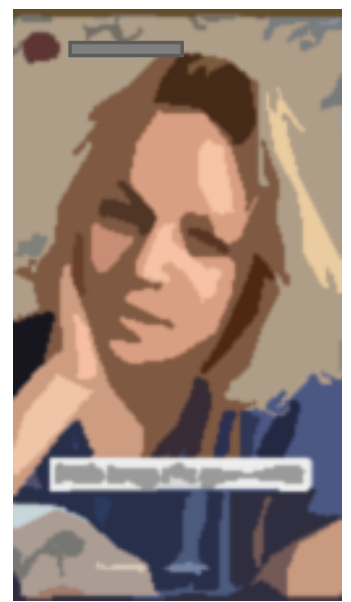
„ehm verschiedene Sachen und seit einigen Jahren, ja, leidet das ein bisschen, mach ich garnichts oder fast nichts ich bin froh, wenn ich mal zum spazieren gehen komme $[\ldots]^{\text {"“ }}$

Abbildung 12: Story Josephine (2020-06-12-22-11 Pos. 37)

Sie thematisiert ihre Unsicherheit, die durch einen bevorstehenden Fahrradausflug mit ihrem Freund entsteht. Nachdem sie sich selbst vor der Kamera Mut zu gesprochen hat, postet sie noch ein Bild ihres Sportoutfits, dass sie sich extra für diesen Ausflug besorgt hat. Im weiteren Verlauf werden die Follower ebenfalls durch ein Bild ihres Fahrrads mit dem Text „Na dann...“ (2020-06-12-22-11 Pos. 37) und ein Selfie von ihr mit „Ich habs geschafft!“ (2020-06-12-2211 Pos. 37) virtuell mitgenommen. Auch nach dem Ausflug meldet sie sich noch ein letztes Mal und beginnt mit „So um die Geschichte jetzt zu Ende zu bringen ehm ich bin jetzt wieder im Auto, mein Freund ist ja mit dem Rad hergefahren, fährt jetzt wieder zurück ehm und ja! Ich habs geschafft [...]“ (2020-06-12-22-11 Pos. 37). Sie scheint also eine Verpflichtung zu spüren, nicht nur ihre Probleme mit ihrer Community zu teilen, sondern auch den weiteren Verlauf und die „Auflösung“ der Geschichte auf Instagram zu posten. Allgemein erweckt dieser Soty beinahe den Eindruck eines therapeutischen Settings, da Josephine sich im Gespräch mit sich selbst Mut zuspricht (zeitlich und räumlich getrennt von den Followern), ihre Gefühle verhandelt und ihre Ängste mitteilt. Der Post fügt sich aber damit auch in ihre „Mission“ ein. Sie versucht auf ihrem Profil Bodypositivity zu leben und vor allem anderen Frauen diese positive 
Einstellung zu ihrem Körper näher zu bringen. Dieser Post ist daher nicht der einzige, in dem sie offen über ihre Gefühle und Unsicherheiten spricht. Dabei rahmt sie diese Geschichten mit der Bodypositivity als Metadiskurs, in dem es okay ist, unsicher zu sein, man sich mit seinem Körper auseinandersetzt sollte, um ihn dann so zu akzeptieren wie er ist.

Die Art und Weise, wie die Stories in der Regel aufgenommen werden, spielt dabei eine große Rolle, wird doch durch das Filmen mit einer Kaffeetasse in der Hand die Situation anders gerahmt als beispielsweise am Schreibtisch sitzend. Dadurch, dass sich Josephine mit einem Kaffee vor die Kamera setzt und über ihre Unsicherheiten spricht, wird eine sehr intime Situation erzeugt, die die Tatsache, dass sie allein zuhause mit ihrem Smartphone sitzt und ein Selbstgespräch mit 4.000 anderen Menschen teilt, in den Hintergrund rückt. Auch Kathi nutzt das vertrauliche Gespräch bei einer Tasse Kaffee sehr oft in ihren Stories. Bei ihr sind meist Produktplatzierungen Teil dieser Stories und sie bindet diese geschickt in Erzählungen über ihren Tagesablauf ein (wie bspw. in Abbildung 11). Die Community lässt sich demnach als eine Art lose, aber vertraute Gemeinschaft beschreiben, die am Alltag der Frauen Anteil hat und wechselseitig die Rolle des Experten und des Schülers einnehmen kann. Im nächsten Abschnitt wird gezeigt, dass sich innerhalb dieser wertschätzenden Fassade vielfach Bewertungspraktiken beobachten lassen und dabei auch Konkurrenz eine Rolle spielt.

\section{Feedback}

Die Beispiele aus Abbildung 11 und 12 zeigen, dass die Kommunikation in den Stories nicht einseitig ist, auch wenn das Format diese Vermutung nahelegt. Mit dem Posten einer Story ist neben der Erstellung und Editierung der Beiträge auch nach dem Teilen zeitlicher Aufwand verbunden. Die öffentlich sichtbare Kommunikation durch Story-Reposts, Tags oder Kommentare ist geprägt von Wertschätzung und gegenseitiger Unterstützung. Hier wird also das Handlungsangebot des Interface weitestgehend angenommen. Inhaltlich bestehen die Kommentare fast ausschließlich aus positivem Feedback oder Bewertungen des Posts. Eine vergleichende Betrachtung zeigt, dass durch die Rahmung des Bildes die Ebene der Bewertung verschoben werden kann. Vollständige Kontrolle haben die Userinnen allerdings nicht. Kathi postet ein Selfie von sich mit dem zentral ins Bild gesetzten ,Sag mir deine Meinung!!“ (Abbildung 13). ${ }^{46}$

\footnotetext{
${ }^{46}$ Kathi postet dieses Bild zweimal: einmal in ihrer Story (Abbildung 13) und einmal in ihrem Feed. Im Feed postet sie das Bild zunächst auch wie auf Abbildung 13 mit der Bildunterschrift, wie in Abbildung 14, einige Wochen später entfernt sie allerdings das Textfeld „New Post“ und „Sag mir deine Meinung!!“, sodass im aktuellen Feed (Stand 06.10.2020) nur noch das Bild ohne Textbausteine sichtbar ist.
} 


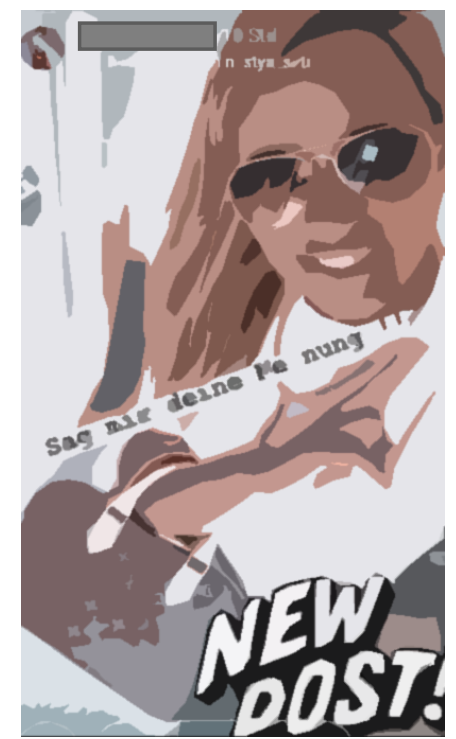

Abbildung 13: Screenshot Kathi (2020-07-03-06-33-02)

Im Foto selbst bleibt zunächst also offen, was Gegenstand der Meinungsäußerung bilden soll. In der Bildunterschrift macht sie dann allerdings deutlich, worum es geht (Abbildung 14).

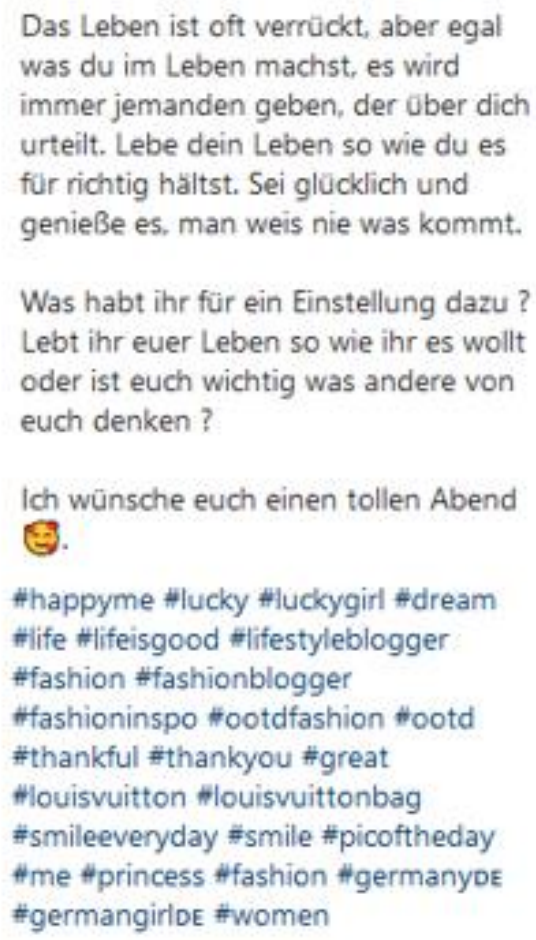

Abbildung 14: Bildunterschrift Post Kathi 02.07.2020 (Screenshot erstellt 06.10.2020)

Sie beginnt ihren Text mit der Aufforderung an die Follower, das Leben zu genießen, und führt in den folgenden zwei Sätzen näher aus, dass man sein Leben so leben soll, wie man es für richtig hält, ohne auf das Urteil von anderen zu zählen. Nachgestellt ist die Frage zu lesen „Was habt ihr für eine Einstellung dazu?“, die noch einmal erläutert wird. Ihren Post schließt sie damit 
ab, dass sie einen tollen Abend wünscht und 26 Hashtags ergänzt. Die offensichtliche Ambivalenz, welche sich aus der Feedback-Aufforderung und der in der Bildunterschrift thematisierten Unabhängigkeit von anderen Meinungen ergibt, wird in den Kommentaren nicht aufgegriffen und erweist sich damit als unproblematisch für die kommentierenden Follower. Authentizität und Einzigartigkeit stehen hier zwar im Fokus, werden aber zwangsläufig durch die Bewertungsaufforderung normiert.

Spannend sind die Kommentare, die sich unter dem Post finden. Manche antworten zwar auf ihre gestellte Frage, der Großteil kommentiert allerdings ihr Aussehen oder ihre Tasche. Das Gesprächsangebot wird nur teilweise angenommen und es findet stattdessen eine Bewertung ihrer selbst statt. Auch wenn die gestellte Frage aufgegriffen wird, sehen sich die Follower offensichtlich dazu aufgefordert, das Selfie einer Bewertung zu unterziehen. Der folgende Kommentar veranschaulicht, welche Gewichtung vorgenommen wird: „Sehe es genau wie du... Steht dir super die Tasche... Braun finde ich auch am schönsten 200 (P) (Post Kathi 02.07.2020).

Diese Kommentarstruktur ist typisch für Kathis Posts und scheint damit verbunden zu sein, dass ihre Selbstdarstellung sehr auf äußere Merkmale und ihr Erscheinungsbild ausgerichtet ist. Das wird deutlich, wenn man als Kontrast Posts von Sabine oder Doris betrachtet. Dort wird die Bildunterschrift eher aufgegriffen und das Verhältnis von oberflächlichen und inhaltlichen Kommentaren kehrt sich um. Doris und Sabine gelingt es also, über ihre Selbstdarstellung die Aufmerksamkeit auf Probleme und Aspekte zu legen, die für sie relevant sind, und durch die Kommentare in den Austausch über diese inhaltlichen Themen zu treten.

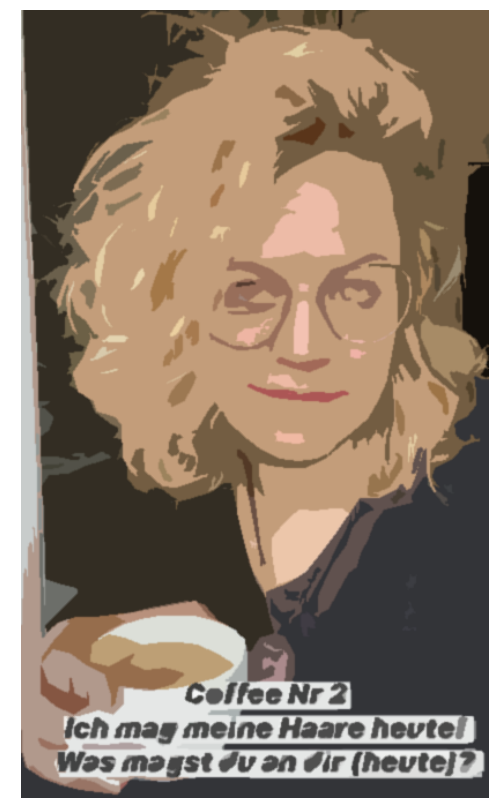

Abbildung 15: Story Josephine (2020-06-18-09-34) 
Ein weiteres Beispiel für eine direkte Bewertungsaufforderung ist ein Story-Post von Josephine. Sie postet ein Selfie von sich. Darunter ist zu lesen „Coffee Nr 2 Ich mag meine Haare heute! Was magst du an dir (heute)?““. Eingeleitet wird der Post durch eine Rahmung des Bildes. Es zeigt sie bei ihrem zweiten Kaffee. Darunter schließt sie eine Bewertung ihrer Haare an. Die Rahmung geht also über die bloße Situationsdefinition hinaus, da sie zusätzlich die Bewertung des Bildes sprachlich vornimmt. Gleichzeitig fordert sie ihre Follower dazu auf, selbst eine situative Selbstbewertung vorzunehmen und mit ihr zu teilen. Da das Selfie in der Story gepostet wurde, sind die Antworten auf ihren Post leider nicht öffentlich sichtbar.

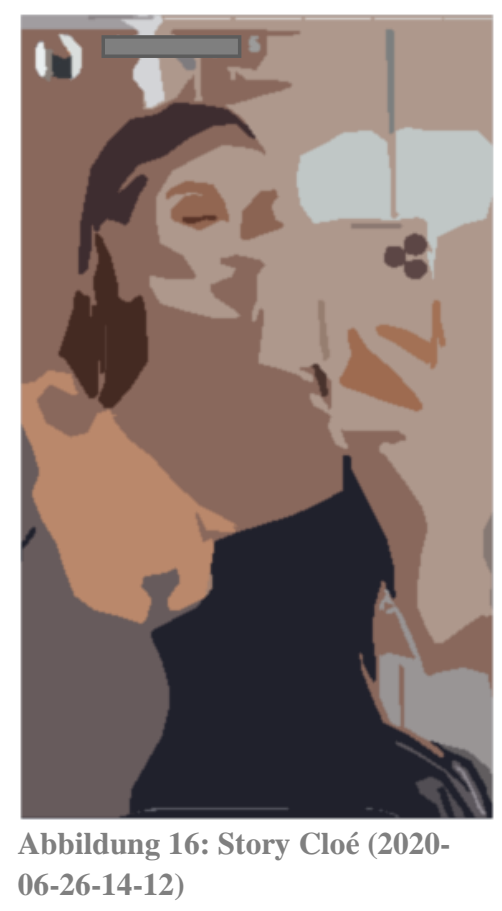

Auch in diesen Beispielen (Abbildung 16 und 17) nimmt die Userin jeweils selbst die Bewertung vorweg. In Abbildung 16 sieht man ein Selfie von Cloé, das in einem Aufzug aufgenommen wurde. Sie streicht sich die Haare aus dem Gesicht und der Blick geht auf den Smartphonebildschirm. In Abbildung 17 ist ein Ausschnitt aus einem Story-Post von Sabine zu sehen. In diesem Video erzählt sie von ihrem bevorstehenden Arztbesuch. Mit „kinda classy, kinda cool“ bleibt Cloé eher unkonkret und ordnet sich nur in ein größeres Feld ein, irgendwie klassisch, irgendwie cool zu sein. Sabine dagegen spricht über ihre bevorstehende Weisheitszahn-Operation und fügt „Mit Filter sieht es ja garnicht so schlecht aus“ eine beiläufige Bewertung ihres Aussehens hinzu. Im Anschluss spricht sie darüber, welche Komplikationen sich für sie bei dieser Operation ergeben und welche Risiken sich durch ihre Erkrankung ergeben. 


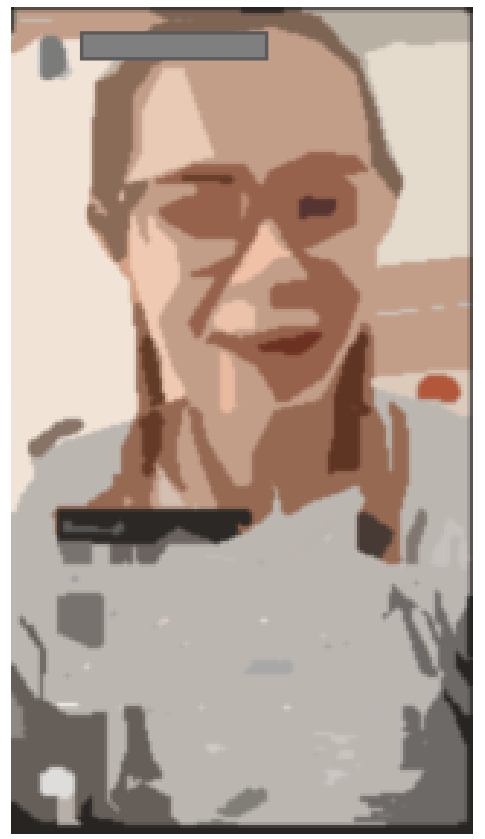

Abbildung 17: Story Sabine (2020-

06-09-15-59 Pos. 40)

An anderen Stellen wird Feedback direkt eingefordert. Es finden sich des Öfteren Umfragen in den Stories, in denen sich die Frauen danach erkundigen, was die Follower gerne als nächstes sehen würden. Sabine und Josephine fragen beispielsweise danach, ob sie Bücherrezensionen in ihr „Angebot“ mit aufnehmen sollen, Doris erkundigt sich nach Vorurteilen, die ihre Follower über behinderte Menschen kennen, um daraus ein Video zu erstellen, das sich mit diesen auseinandersetzt. Kathi erkundigt sich danach, ob ihre Community ein Bild ihres Stand-UpPaddle-Ausflugs sehen möchte, oder ob sie Tofu genauso mögen wie sie. ${ }^{47}$ Auch an anderen Stellen werden Posts mit „Eure Meinung interessiert mich“ oder ähnlichen Feedback-Aufforderungen versehen und zeigen, dass die Community aktiviert wird, gepostete Inhalte (seien sie inhaltlicher oder ästhetischer Natur) zu bewerten und evaluieren.

Auch von außen werden Bewertungen an die Frauen herangetragen, zum Beispiel, indem sie von anderen User*innen in deren Story vorgestellt werden. Dabei werden sie meist als inspirierender, vorbildhafter, oder anderweitig interessanter Account gerahmt, dem es zu folgen lohnt. Teilweise wird Sabine auch durch andere in eine Art Bestenliste eingeordnet. So teilt eine Userin eine Liste an favorisierten „Alltagshelden“ und vergibt Bestenlistenplätze in Kate-

\footnotetext{
${ }^{47}$ Auf diese Frage folgt eine Werbeeinspielung für Tofu und eine Aufzählung vielfältiger Verwendungsweisen des Sojaprodukts. Die Frage kann hier gedeutet werden als ein Ausloten der Konsuminteressen ihrer Follower. Die Umfrage nach der Beliebtheit von Tofu geht eher zu Ungunsten desselben aus und während sie andere Produkte desselben Herstellers öfter bewirbt, bleibt es bei dieser einen Werbung für Tofu.
} 
gorien wie Bodypositivity und Kämpferherz, aber auch ein kreativer Kopf wird dadurch ausgezeichnet. Sabine wird unter der Kategorie Inspiration aufgeführt. Teil der Daten wird diese Liste dadurch, dass Sabine sie in ihrer Story repostet und sich für die Auszeichnung bedankt.

Die Bewertung nahezu aller geteilten Inhalte ist damit fest in die alltäglichen Handlungspraktiken eingebettet und wird kaum hinterfragt. Der Rahmen der wertschätzenden Community erlaubt dabei die Bewertbarkeit aller bildlichen und textlichen „Lifelogs“ der Frauen und die Urteile über den geposteten Content werden an vielen Stellen direkt eingefordert.

\section{Und du so?}

Es ist zu vermuten, dass Vergleiche mit geposteten Inhalten und dem eigenen Leben bei den User*innen ständig stattfinden und keiner gesonderten Rahmung benötigen, ebenso wie die Bewertung der Bilder keiner gesonderten Aufforderung bedarf. Trotzdem finden sich in den Daten viele Vergleiche, die über die Stories oder im Feed verhandelt werden. Durch die Darstellung der eigenen Aktivitäten und einer anschließenden Frage, was die Follower gerade tun, wird aktiv ein Vergleich erzeugt. Das Vergleichskriterium bleibt dabei oft implizit, wird aber auch häufig durch Hashtags oder in anderer Form sprachlich markiert. Dabei reicht es, ein Selfie zu posten und andere Nutzer*innen darauf zu verlinken, um einen direkten Vergleich zu erzeugen. Vor allem Kathi nutzt diese Form des Vergleichs durch Vernetzung, wobei sich die markierten Personen des Öfteren dem Vergleich entziehen. Durch Aussagen wie „Mit meinem Speckpack kann ich eh nicht mithalten“ entzieht sich so beispielsweise eine Frau, die in einem Post von Kathi markiert wurde, auf dem man, wie so häufig, ihr definiertes Sixpack sieht. 


\section{Ästhetische Inszenierung des eigenen Lebens}

Der erste Abschnitt hat deutlich gemacht, dass Instagram nicht nur eine Plattform ist, um sich selbst darzustellen, sondern auch einen Ort vielseitiger sozialer Interaktionen bietet. Dennoch spielt die optimale Inszenierung des alltäglichen Lebens eine große Rolle in den Daten.

Oft im Fokus der Kamera stehen die Frauen selbst, sei es in Form von Selfies vor dem Spiegel, kurzen Videos in den Stories oder auch Bilder aus (professionellen) Shootings. Aber auch Land-

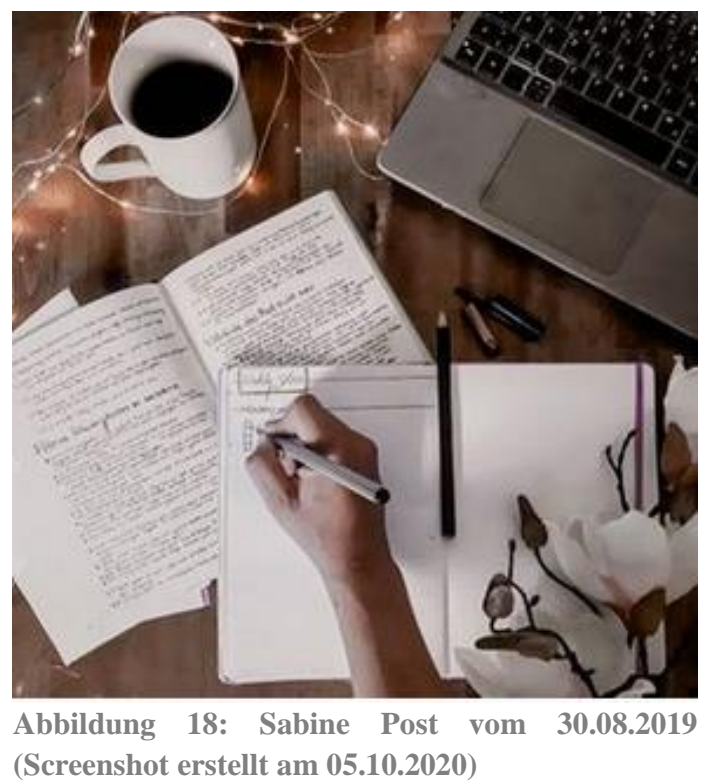

schaften und Gegenstände des alltäglichen Gebrauchs finden Einzug in die Posts. Die Bedeutung, die der ästhetischen Darstellung dieser alltäglichen Situationen zukommt, wird in Abbildung 18 besonders deutlich.

Man sieht eine Kaffeetasse, die Tastatur eines Laptops, zwei Notizbücher und eine Hand, die dabei zu sein scheint, die Notizen zu ergänzen. Um die Kaffeetasse herum liegt eine Lichterkette. Durch die Posts alltäglicher Gegenstände wird deutlich, dass nicht der Gegenstand als solcher instagramabil ist, sondern vielmehr die ästhetische Inszenierung desselben. Sehr selten wird ein Gegenstand schmucklos gepostet. Auf anderen Bildern werden die Gegenstände ebenfalls durch Blumen, Tannenzapfen, Kerzen oder ähnliches ergänzt. Die Aushandlung dessen, was als ästhetisch gilt, findet weitestgehend über sehr bekannte Influencer*innen oder auch verschiedene Trends statt. Oftmals dienen andere Posts oder Bilder aus anderen sozialen Medien als Inspiration für Bilder oder Bildkompositionen. Ähnlich zu den Ergebnissen von Stefanie Duttweiler scheint die Bedeutung nicht in den Dingen selbst zu liegen, sondern in diesem Fall durch die Ästhetisierung derselben relevant zu werden. Ähnlich wie Selbstvermessungs- 
praktiken dazu in der Lage sind, alltäglichen Handlungen Wert zu verleihen, scheint die ästhetische Inszenierung des Alltags dazu in der Lage zu sein, Gegenständen eben durch diese Darstellung und das Teilen auf Instagram Bedeutung zu geben (vgl. Duttweiler 2018). Die doppelte Bedeutungszuschreibung, wie sie Duttweiler herausarbeitet, ist also nicht auf quantitative Selbstthematisierung beschränkt, sondern findet sich auch in den in Instagram generierten Daten.

Likes und Kommentare gehen einher mit der Veränderung des Blicks auf die eigenen Handlungen und die Umwelt. Viele Nutzer*innen in Sascha Oswalds Sample beschreiben die Ausrichtung des Alltags anhand ästhetischer Standards. Auch wenn nicht alle ihre Umwelt bewusst beeinflussen, um sie an die auf Instagram vermittelten Anforderungen anzupassen, wird die Umgebung zumindest vermehrt auf ihre Fotogenität (respektive Instagramabilität) hinterfragt.

Aber auch umgekehrt berichten die Nutzer*innen davon, dass sie ihre Mitmenschen anders wahrnehmen und beurteilen, und versuchen aufgrund von Likes bestimmter Personen, Rückschlüsse auf deren Persönlichkeit zu ziehen (vgl. Oswald 2019, 4). Diese Erkenntnisse können erklären, wieso Bilder in den Daten selten „schmucklos“ zu sehen sind. Gegenstände, aber auch die Frauen selbst werden oft in irgendeiner Weise dekorativ in Szene gesetzt, wenn beispielsweise eine Tasse mit Pudding durch eine Sonnenblume ergänzt werden oder Doris ihre Haare mit Blumen verziert (Abbildung 19). ${ }^{48}$

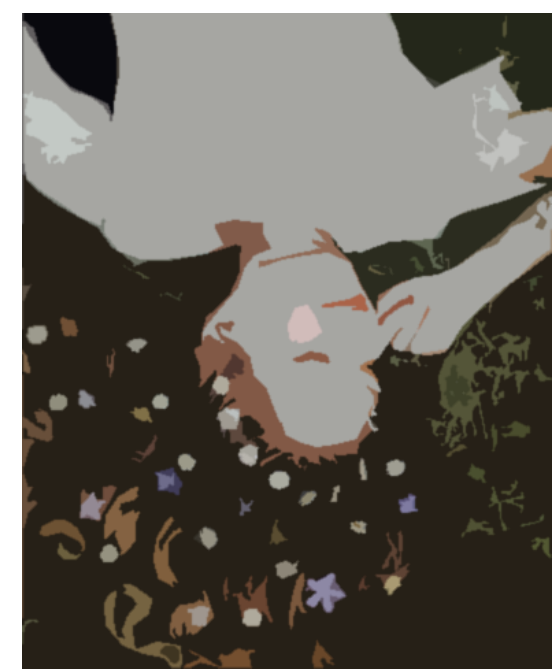

Abbildung 19: Doris Post (Screenshot erstellt am 18.09.2020)

\footnotetext{
${ }^{48}$ Für die Arbeit wurden teilweise besonders deutliche oder plakative Beispiele ausgewählt. So ist diese sehr aufwendige Inszenierung nicht unbedingt typisch für Doris Profil, in anderen Bildern hält sie etwa einen Blumenstrauß in der Hand oder fügt kleine Lichtkugeln in das Foto ein. Die Inszenierung und Dekoration des Dargestellten kann sehr oft im Datenkorpus beobachtet werden, häufig allerdings auch in weniger aufwendigerer oder subtilerer Form.
} 
Darüber hinaus lassen sich bestimmte Motive beobachten, die sich in den Profilen immer wieder wiederholen und eine gute Ausdrucksmöglichkeit $\mathrm{zu}$ bieten scheinen. Darunter sind \#coffee- und \#goodmorning-Beiträge zu fassen. Es ist auffällig in den Daten, wie häufig über vier der fünf Profile hinweg diese beiden Narrative aufgegriffen werden. Kaffeetassen und ein schlichtes „good morning“ scheinen eine gute Gelegenheit zur Selbstdarstellung zu bieten, und sich gleichzeitig auch besonders gut dazu zu eignen, eine intime und vertraute Atmosphäre zu kreieren. Der Kaffee dient als Symbol für Ruhe und Entspannung und weckt die Assoziation man würde mit einer guten Freundin einen Kaffee trinken. Es ist außerdem naheliegend, dass durch den allgemeinen Charakter die beiden Hashtags mit einer hohen Reichweite assoziiert werden. So wurden unter dem Hashtag \#coffee zum Zeitpunkt der Arbeit bereits 127 Millionen Beiträge und unter \#goodmorning 119 Millionen Beiträge registriert. Im Vergleich dazu liegt der Hashtag \#bodypositive mit 13 Millionen weit zurück.

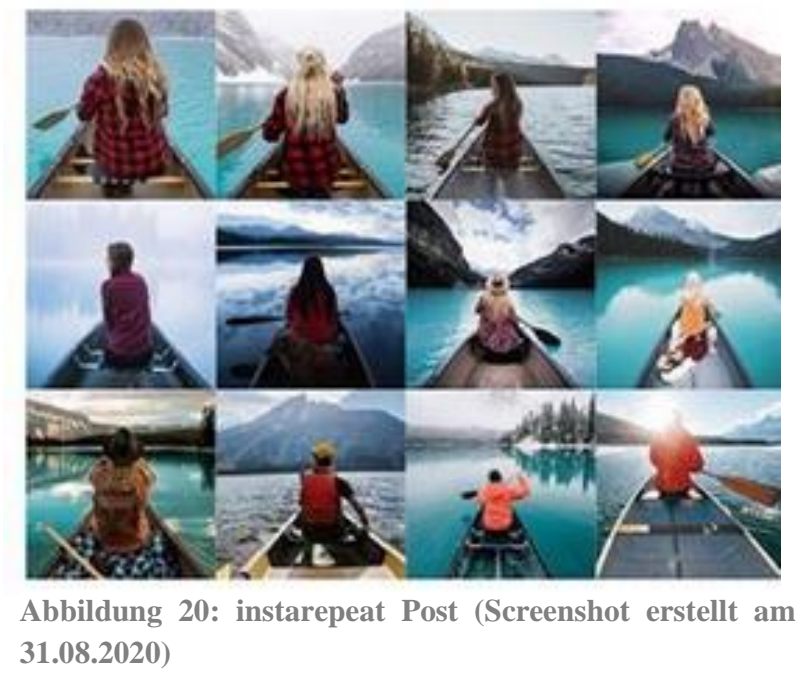

Die starke Macht der Konventionen über die ästhetische Funktion zeigt sich in einer Art Metaaccount (\#instarepeat), der regelmäßig beliebte Motive auf Instagram sammelt und sie zu einer Collage zusammenfügt. Dadurch wird dem Betrachter oder der Betrachterin vorgeführt, wie ähnlich sich die Fotografien einzigartiger Ausflüge und Erlebnisse oft sind (siehe Abbildung 20). Dem Streben nach Einzigartigkeit und Individualität stehen also Nachahmungs- und Konformitätstendenzen gegenüber. Treiber dieser Konformität sind vermutlich die Likes. Erreicht ein Post viele Likes, wird es oft vielfach nachgestellt in der Hoffnung, mit diesem Bild ebenfalls eine hohe Resonanz zu erzeugen. ${ }^{49}$

\footnotetext{
${ }^{49}$ In diesem Kontext erzählt eine Youtuberin von ihrem Erfolg auf Instagram und beschreibt, dass sie viele virale Inhalte durch Nachahmung generiert hat. Sie nennt als Beispiel die Beobachtung, dass Bilder mit einem speziellen
} 
Die Welt, die durch diese ästhetische Inszenierung erschaffen wird, wird nur selten in den Kontrast zur Realität gestellt. Oft wird der Eindruck erweckt, es handle sich um authentische und spontane Eindrücke aus dem Alltag der Frauen. Die Editierung, Inszenierung und das Arrangement des Körpers und verschiedener Gegenstände wird kaum sprachlich verhandelt.

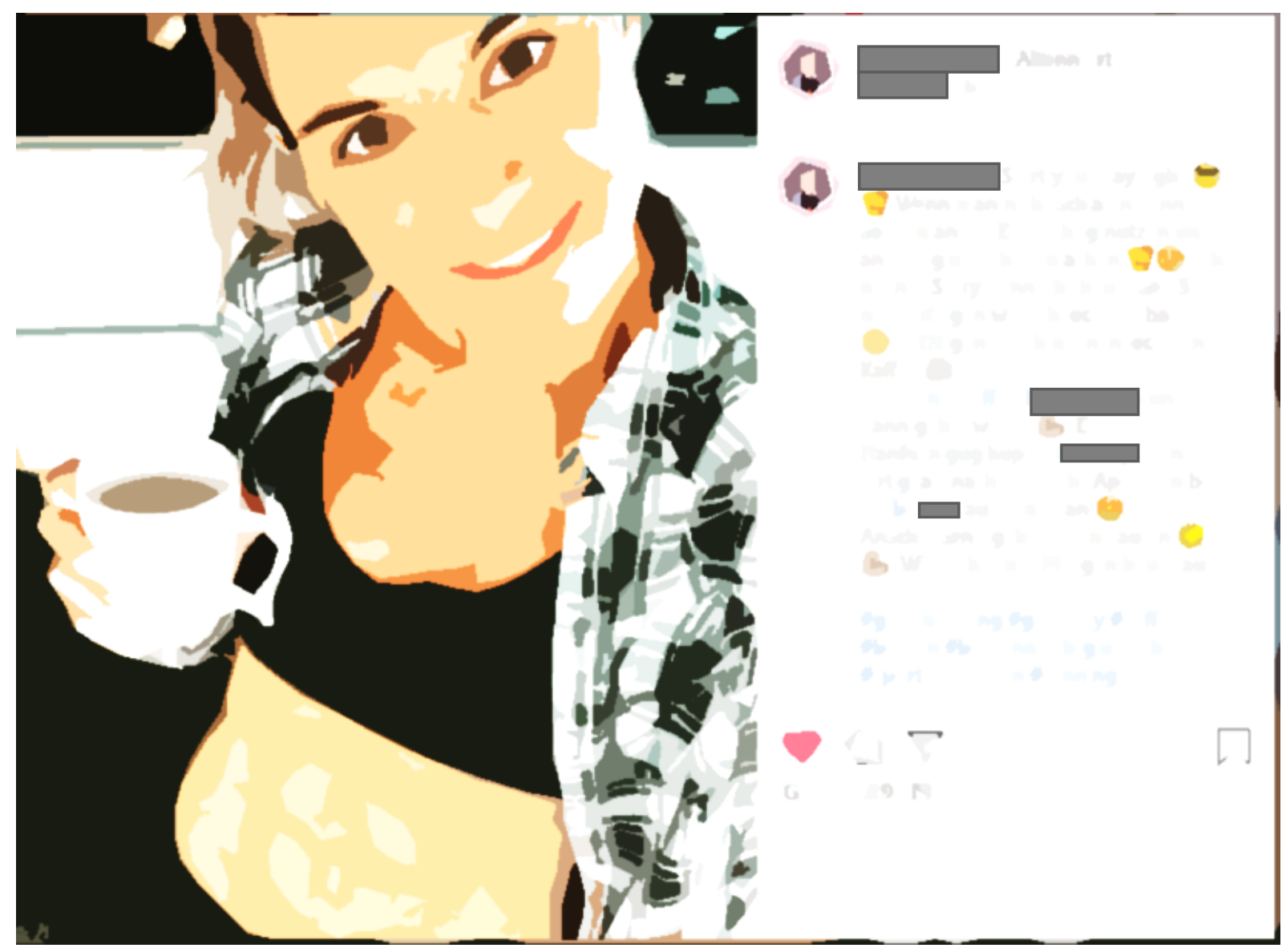

Abbildung 21: Kathi Post (2020-02-08-09-45)

Beispielhaft dafür steht Kathis Post vom 3. Juni (Abbildung 21). Sie inszeniert sich als Powerfrau, die neben ihrer täglichen Care-Arbeit und dem straffen Sportprogramm noch Zeit zu Backen und gemütlichen Kaffeetrinken hat. Sie erzählt den Followern, dass sie seit 5 Uhr morgens auf den Beinen ist, was sie alles vorhat und bindet ihre Kooperationspartner geschickt in ihre Erzählung ein. Auf dem Selfie sieht sie erholt und frisch aus, das Make-Up sitzt, die Bluse ist zurückgeschlagen, um das Sixpack der Kamera zu offenbaren.

Ästhetik spielt also nicht nur im Design der App eine Rolle, sondern findet sich auch im generierten Content der untersuchten Frauen. Drei der fünf Untersuchten verwenden standardmäßig in ihrer Story Filter. So wird die Haut je nach Profil sonnengebräunt, weicher, ebenmäßiger,

braunen Mantel auf unterschiedlichen Profilen eine hohe Likezahl erreichten und sie sich deshalb auch einen ähnlichen Mantel gekauft hat (https://www.youtube.com/watch?v=plCd4RLr8Kk\&list=PLbzreVHZyic4EYPkaPEMz7Gu2u3zw_ihH\&index=7; zuletzt aufgerufen am 04.10.2020). 
mit nostalgischen Mustern verziert oder im Extremfall mit dutzenden blinkenden Diamanten versehen. ${ }^{50}$ In den Stories werden die Filter in Echtzeit auf die Gesichter und den Hintergrund projiziert und die angewendeten Filter werden lediglich klein oben am Bildrand erwähnt. Im Feed weitete sich die Verwendung von Bildbearbeitungsfeatures aus. Lediglich Josephine thematisiert regelmäßig die Diskrepanz, die sie zwischen Instagram und der Realität beobachtet. In ihren Posts und Stories lässt sich eine bewusste Abgrenzung von dem Bild, das Instagram vermittelt, und der Realität beobachten. Durch zeitliche oder szenische Vergleiche, aber auch durch das Posten von Bildern, die ihrer Meinung nach nicht dem auf Instagram vermittelten Ideal entsprechen, möchte sie diese Differenz sichtbar machen. Diese Strategie ist Teil ihres Empowerments und der Weitergabe ihrer positiven Körperwahrnehmung und Selbstliebe. Auf Nachfrage, welche Bedeutung Zahlen in Form von Likes für sie haben, antwortet sie, dass sie es schätzt, wenn ihre Posts gut ankommen. Sie erzählt aber auch, dass sie Bilder postet, von

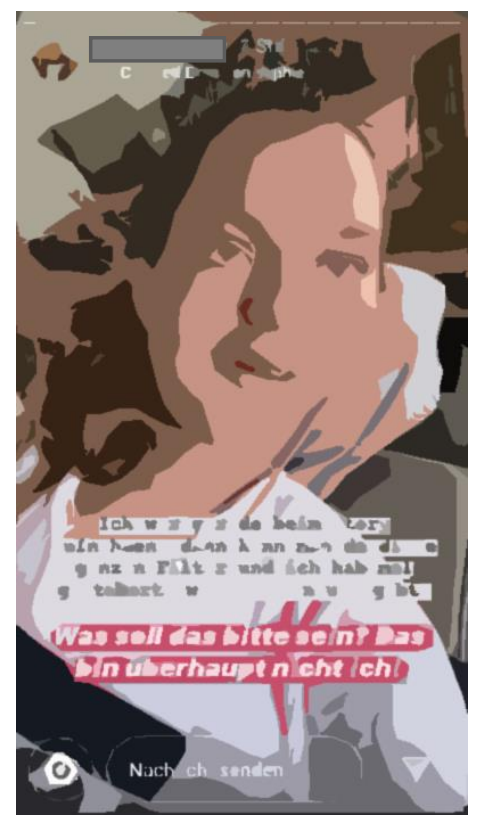

Abbildung 22: Story Doris (202005-24-18-56-15)

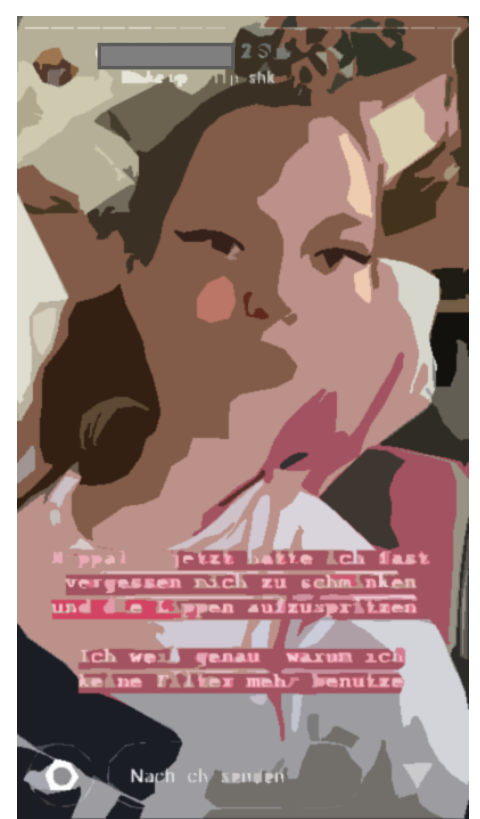

Abbildung 23: Story Doris (202005-24-18-56-21)

denen sie weiß, dass sie wenige Likes erhalten, ihr aber die Botschaft wichtiger ist als das positive quantitative Feedback. Auch Doris spricht sich in einer ihrer Stories aktiv gegen die Verwendung von Filtern aus und diskutiert vor der Kamera, welche Meinung sie dazu hat:

Sie spricht darüber, wie sehr ihrer Meinung nach Filter die Wahrnehmung beeinflussen. Man würde sich ohne Filter nichtmehr wohl fühlen und diesen Trend findet sie bedenklich. Sie spielt

\footnotetext{
${ }^{50}$ Eine Taktik, die im Zuge der Strategisierung des Instagram-Profils empfohlen wird, ist die Verwendung eines Filters in allen Stories und auch eine ähnliche Bearbeitung der Posts im Feed. Diese Taktik verwenden - ob bewusst oder unbewusst - Kathi, Cloé und auch Sabine. Dadurch, dass das Gesicht der Frauen immer gleich verändert wird, wird die Verwendung des Filters zusätzlich verschleiert.
} 
verschiedene Filter durch und ironisiert die verschönernde Wirkung derselben. „Hoppala, jetzt hätte ich fast vergessen mich zu schminken und die Lippen aufzuspritzen. Ich weiß genau, warum ich keine Filter mehr benutze“. Danach wechselt wieder auf die „normale“ Ansicht. Für eine Story ungewöhnlich lange verhandelt sie daraufhin eingehender die Problematik, die sie auf Instagram sieht. Sie habe nichts gegen Menschen, die sich bräunen, ihre Lippen aufspritzen oder ähnliches, nur die Verwendung der Filter finde sie schwierig. Durch den täglichen Konsum werde man fortlaufend mit „perfekten“ und „gleichen“ Gesichtern konfrontiert, dies sei aber nicht die Realität.

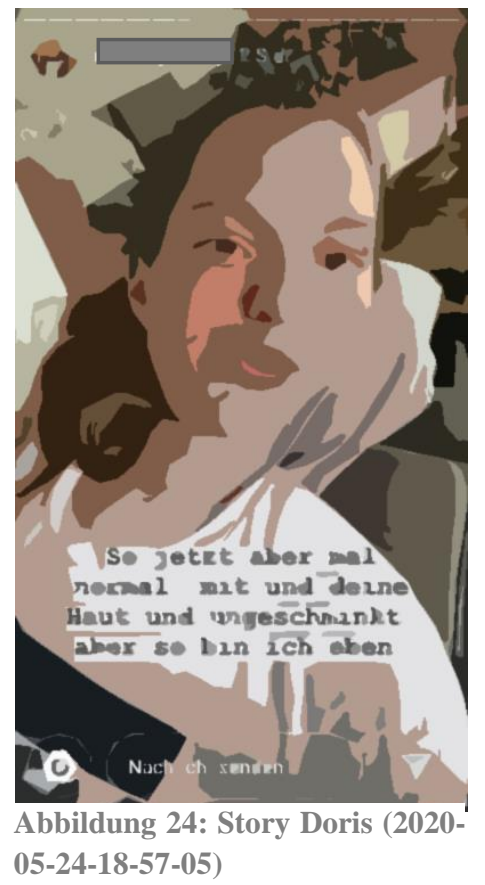

Nach einiger Zeit postet sie einen Screenshot einer ihr zugesendeten Nachricht, die ihr zustimmt und ebenfalls problematisiert, dass durch die perfekte Inszenierung auf Instagram „viele sich nicht schön genug finden“. Doris stellt daraufhin klar, dass auch ihre Bilder im Feed bearbeitet seien, Fotografie und die Bildbearbeitung sei ein Hobby, die sie aber klar von Situationen im Alltag abgrenzt. Sie markiert damit eine Differenz zwischen den ,,alltäglichen“ Stories und den „künstlerischen“ Bildern im Feed. In der Story würde sie niemandem etwas vormachen wollen und vergleicht die Aufnahme der Story mit einem Treffen auf der Straße, dort würde sie ,halt nur mal so aus[sehen]““ (das Satzpartikel „,mit und deine Haut“ korrigiert sich später, gemeint ist „mit unreiner Haut“). Sie schließt ihre Ausführungen ab: „Aber das bin eben ich. Wisst ihr, was ich damit sagen will?“ (Abbildung 24). Diese klare Herausarbeitung der Differenz zwischen dem realen Aussehen und dem, das auf Instagram gezeigt wird, bildet eine Ausnahme in den Daten. 


\section{Markt der Aufmerksamkeit}

Durch die Konsumlogik, welche durch die Gestaltung der App erzeugt wird, finden sich die Nutzerinnen vor die Herausforderung gestellt, die Aufmerksamkeit der Follower zu erregen und aus dem unendlichen Katalog der anderen Posts herauszustechen. Teilweise werden dafür klassische Methoden des Marketings verwendet, wie zum Beispiel das Einfügen blinkender, sich bewegender Elemente in die Stories. Oft werden aber auch neue Posts ebenfalls in der Story gepostet, nur dass im Gegensatz zu den ,richtigen“ Posts meist Teile des Bildes abgedeckt werden, wie beispielweise in Abbildung 25 zu sehen ist. Über das Bild fügt Cloé noch den Satz „Tap and show some love $<3$ “ und verbindet damit die Neuigkeit über ihren neuen Post mit einer direkten Aufforderung, ihr Bild mit einem Like zu versehen.

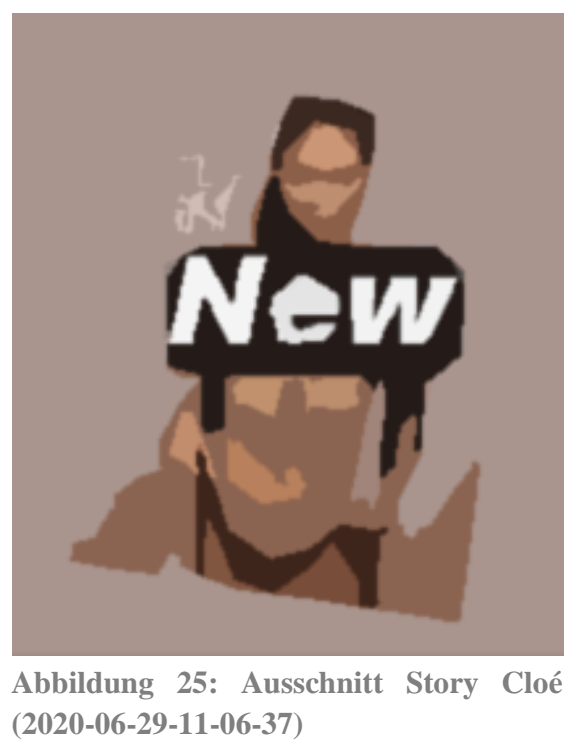

Der Markt, in den sich die User*innen durch das Posten von Bildern einordnen, wird oft implizit verhandelt, zeigt sich aber auch teilweise ganz offen. Kathi versieht ihr Post häufig mit „Save to support me“ oder „Gerne speichern“. Mit der Aufforderung, ihre Posts zu speichern, wird der Versuch markiert, eine höhere Sichtbarkeit zu erzielen. ${ }^{51}$ Posts, mit denen häufig interagiert wird, sei es durch Likes, Kommentare oder Speichern, werden eher in den Feeds anderer Nutzer*innen gezeigt als Posts, die weniger Reaktionen hervorrufen. ${ }^{52}$ Möchte man sich auf dem Markt der Aufmerksamkeit behaupten, ist es demnach wichtig, eine hohe Sichtbarkeit durch die Art und Weise des Postens zu generieren. Kathi ergänzt ihre Kommentare häufig durch

\footnotetext{
${ }^{51}$ Allgemein gilt die Auffassung, dass hoher „Traffic“, d.h. eine hohe Fluktuation, auf dem eigenen Profil den Algorithmus dazu veranlasst, diese Bilder eher im Feed anderer Profile anzuzeigen als Posts, die nicht häufig kommentiert oder geliked werden.

${ }^{52}$ Auch die Verwendung vieler beliebter Hashtags ist eine Strategie, um die eigene Sichtbarkeit und Reichweite zu erhöhen. Ratgeber für Influencer*innen heben ebenfalls immer wieder hervor, dass es wichtig ist, in den Posts Einladungen zur Interaktion zu geben, da Fragen an die Follower mehr Kommentare und dadurch eine höhere Engagementrate und Sichtbarkeit erzeugen.
} 
Fragen an ihre Community und vor allem in der Story findet in vielen Fällen eine direkte Ansprache an die Follower statt.

Kathi erzählt auf Nachfrage durch die Forscherin, dass sie ihr Profil derzeit von einem Hobby hin zu einer professionelleren Verwendung verschiebt. Vor allem in ihrem Profil lassen sich verschiedene Strategisierungsprozesse beobachten, die diese Verschiebung deutlich machen. Auf die Nachfrage, welche Rolle Zahlen auf Instagram spielen, schreibt sie diesen keine primäre Funktion zu. Zwar würden sich viele Likes „,natürlich“ positiv auf die Kooperationen auswirken, im Vordergrund steht für sie aber die soziale Anerkennung, für welche die Likes einen Indikator bilden. ${ }^{53}$ Auf dem Weg zu mehr Reichweite lässt sich bei Kathi nachvollziehen, wie sie ihr Postingverhalten an ihre Follower angepasst hat. Bilder, auf denen ihr Sixpack zu sehen ist, erreichen tendenziell mehr Likes als Bilder, die sie vollständig bekleidet oder mit Freunden zeigen. So lässt sich in ihrem Feed beobachten, dass Fotos aus einem anderen Kontext längerfristig mehr und mehr Selfies von ihr und ihrem trainierten Bauch weichen. Die Muskeln sind ihr Kapital und so treten diese immer weiter in den Vordergrund und werden nach und nach zur Werbefläche für Fitnessriegeln und Kaffee (Abbildung 26 und 27).

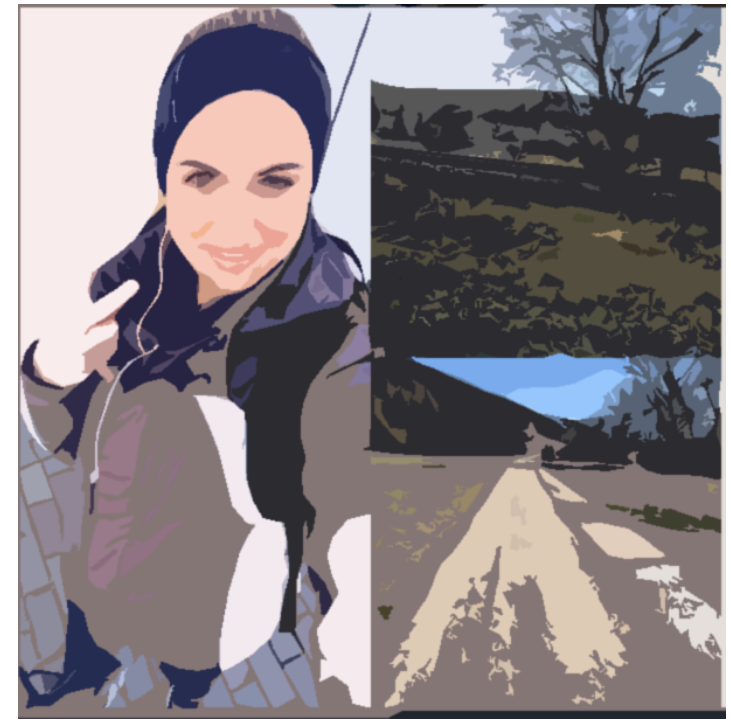

Abbildung 26: Post Kathi 20.03.2019

(Screenshot erstellt am 02.08.2020)

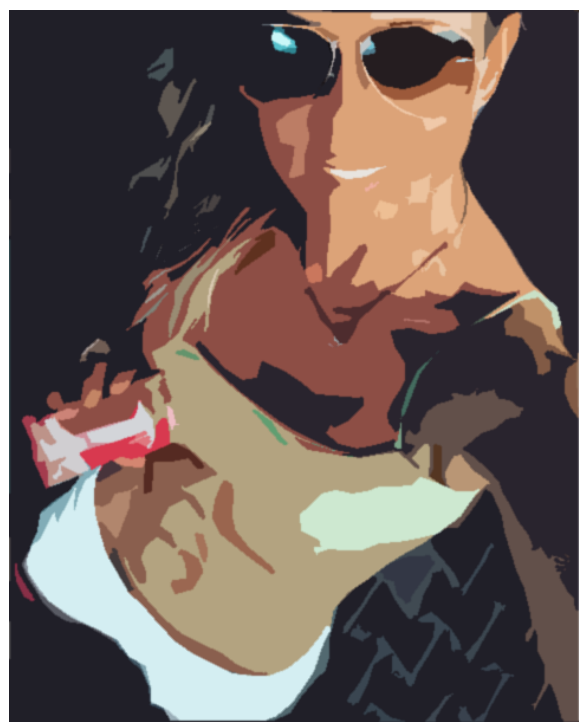

Abbildung 27: Post Kathi 31.07.2020 (Screenshot erstellt am 02.08.2020)

\footnotetext{
${ }^{53}$ Die Bedeutung der Likes als Indikator für soziale Anerkennung deckt sich mit den von Sascha Oswald durchgeführten Interviews. So beobachtet er eine affektive Verschränkung und Symbiose der Like-Zahl und dem mentalen Selbstbild (vgl. Oswald 2019, 5).
} 
Um trotz dieser zunehmend professionellen Inszenierung und der Konstruktion des perfekten „Instalifes“ authentisch zu bleiben, integriert sie gezielt Brüche in ihre Stories. Auch bei Cloé lassen sich ähnliche, vereinzelte Ausbrüche aus der konstruierten Welt beobachten, wenngleich diese in einem gewissen Rahmen bleiben. So postet Cloé ein Bild, das sie als erschöpftes Selfie im Zug rahmt. Auch wenn ihre Erschöpfung und das von ihr herausgehobene erschöpfte Aussehen Thema des Selfies ist, verzichtet sie nicht auf ihren üblichen Filter. Im Gegensatz zu Doris hält Cloé damit an dem editierten Bild ihrer selbst fest.

Diese gezielten Brüche zeigen den doppelten Anspruch, den die Nutzerinnen auf Instagram an sich stellen. Zum einen muss das Leben so perfekt und makellos wie möglich beschrieben werden, auf der anderen Seite darf auf dem Weg zur Perfektion die Authentizität nicht verloren gehen. Die Tatsache, dass Bilder im Allgemeinen und Selfies im Besonderen gut dazu geeignet sind, die Aufmerksamkeit anderer Nutzer*innen zu erwecken, wird teilweise von den untersuchten Frauen gezielt genutzt. So steht manchmal das Bild wenig in Zusammenhang mit dem eingefügten Untertitel. Die Frauen generieren also zunächst durch das Posten eines ästhetischen Selfies Aufmerksamkeit und nutzen diese, um ihre Botschaft an die Follower weiterzugeben. Sehr deutlich wird das bei Sabine und Doris. Beide posten oft Bilder (auf denen zumeist sie selbst zu sehen sind) und sprechen dann in den Untertiteln über ihr Leben mit Handicap. Beispielsweise postet Sabine ein Bild von sich und spricht dann über ihre Angst vor einer CoronaInfektion und versucht, ihre Follower für die Gefahren zu sensibilisieren, denen sich Menschen ausgesetzt fühlen, die zur Risikogruppe zählen.

\subsection{Vergleichen und Bewerten auf Instagram}

Die Darstellung der Ergebnisse hat gezeigt, dass Instagram ein Ort vielschichtiger Vergleichsund Bewertungspraktiken ist, der durch die digitale Selbstdarstellung und Kommunikation über diese Inszenierung entsteht. In diesem Kapitel werden nun wichtige Aspekte aus den Daten herausgegriffen und näher verhandelt.

\section{Instagramability}

Ein zentraler Begriff, der sich in der Untersuchung herauskristallisiert hat, ist der der Instagramabilität. Dabei bezeichnet er zunächst nur die Eigenschaft eines Objekts (oder Subjekts, wobei die Grenzen hier zu verschwimmen scheinen), für Instagram ein geeignetes Fotomotiv zu sein. Ob etwas instagramabil ist oder nicht, wird nicht von außen durch ein Regelset, eine Autorität oder Ähnliches definiert. Das Interface legt zwar ein gewisses Deutungsschema nahe, nämlich durch Filter editierte Schnappschüsse, dennoch ist nicht von außen festgesetzt, was als teilbar 
angesehen wird. Deshalb ist die Instagramability Gegenstand von Aushandlungen und interaktiven Prozessen.

Auch wenn die Instagramability als Maßstab für die Bewertung der Bilder angelegt wird, erkennt man bei genauerer Betrachtung, dass keine einheitliche Auffassung davon existiert, was als teilbar eingestuft wird. Die erste wichtige Dimension, die darüber entscheidet, was als instagramabil gilt, bildet die Resonanz, die ein Post erzeugt. Diese Resonanz funktioniert vor allem über die quantitativen Likes und schriftlichen Kommentare. Posts mit vielen Likes erzeugen Nachahmer und ähnliche Posts, wodurch in einer Art selbstverstärkenden Spirale sich gewisse Vorstellungen instagramabiler Darstellungen zum einen weitläufig verbreiten, zum anderen aber auch aneinander angleichen. Ergebnis sind vermeintlich einzigartige Bilder, deren Einzigartigkeit aber zwangsläufig mit einer hohen Rezeption und Nachahmung verloren geht. Da aber das Herausstechen aus der Masse und die Herausarbeitung der eigenen Individualität und Authentizität essentiell für die Gewinnung und Bindung der Community sind, finden sich die User*innen mit einer gewissen Steigerungslogik konfrontiert, der sie folgen müssen, um im Markt der Aufmerksamkeit ihren Wert zu halten. In diesem Fall scheint die von Rosa beschriebene Beschleunigung in den Subjekten anzugelangen und Sascha Oswald spricht von einer dynamischen Ich-Stabilisierung, die sich auch auf Instagram beobachten lässt (vgl. ebd., 9).

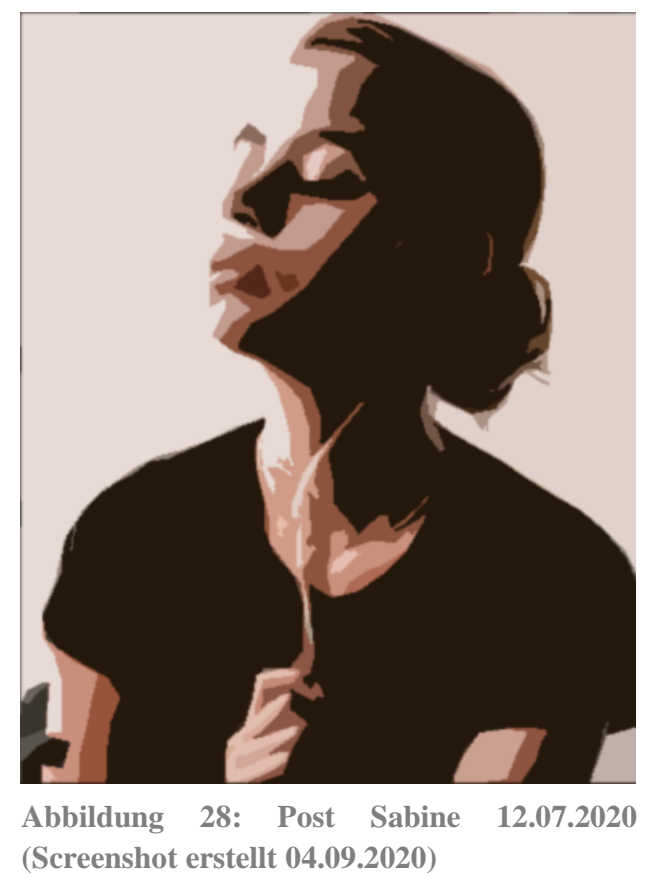

Durch Bilder wie Abbildung 28 wird eine zweite Dimension deutlich: Ästhetisch inszeniert, weiten einige der Frauen den Bereich des Normalen aus und erweitern das gängige Schönheitsideal. Sabine inszeniert sich zusammen mit ihr Beatmungsschlauch und setzt diesen mit der Berührung ihrer Hand in Szene. Darüber hinaus können sich die User*innen gezielt über dieses 
Bewertungsschema hinwegsetzen und innerhalb ihrer Community eigene Werte definieren. Die Verhandlung dessen, was als teilbar erachtet wird, findet also auch über verschiedene Inhalte statt - wenn Josephine beispielsweise gezielt ungeschönte Bilder postet, um sich vom perfekten „Instalife“ abzugrenzen und für sich selbst an einer positiven Körperwahrnehmung zu arbeiten.

Vor allem vor dem Hintergrund der Ökonomisierung erfolgreicher Nutzer*innen gewinnt die perfekte und doch authentische Inszenierung des Alltags große Bedeutung. Bei der Analyse solcher Posts lassen sich gewisse Parallelen zu den Ergebnissen finden, die Erving Goffman bereits in den späten 1970er Jahren für die Konstruktion von Werbung in klassischen Medien herausgearbeitet hat. Mit „kommerziellem Realismus“ beschreibt er die erfolgreiche Rahmung einer inszenierten Szene als spontanen Ausschnitt des Alltags (Goffman 1981). In den Stories der untersuchten Mikroinfluencerinnen wird dies zum Teil perfektioniert und die Produktplatzierung geschickt in Erzählungen über den Alltag eingebunden. Für den Betrachter oder die Betrachterin wird es dadurch fast unmöglich zu unterscheiden, an welcher Stelle die Werbung beginnt und die Selbstdarstellung endet.

Neben der Grenze zwischen Privatheit und Ökonomie verschwimmt auch die Grenze zwischen dem ,realen“ und dem editierten Selbst. Dadurch, dass sich die Frauen selbst jeden Tag mehrmals in geschönter Form online präsentieren und die Follower nur dieses Bild zu Gesicht bekommen, wird es schwieriger, diese Inszenierungen von der Realität abzugrenzen. Dass diese Unterscheidung relevant für die Nutzer*innen ist, zeigt nicht nur die bestehende Literatur (Araújo et al. 2014; Caldeira, Ridder \& van Bauwel 2020), sondern lässt sich auch an den Posts von Josephine und Doris ablesen. Es deutet also alles darauf hin, dass die Instagramability den (sozialen) Blick auf die Welt verändert, und es stellt sich die Frage wem oder was Wert innerhalb dieses Maßstabes verliehen wird.

\section{Vergleichbarkeit}

Dadurch, dass die Posts auf Instagram geteilt werden, klassifizieren sie sich automatisch als instagramabilen Post (im wörtlichen Sinne), und die Tatsache, dass dieses Kriterium als Vergleichskriterium herangezogen wird bzw. keine ergänzenden Gleichheitsunterstellungen benötigt, zeigt die produktive Macht, die das Interface hier entfaltet. Durch sprachliche Rahmungen, wie beispielsweise \#mummys oder \#chronicalillness, können zwar zusätzliche Vergleichskriterien eingeführt werden, prinzipiell kann aber das Gezeigte immer unter dem Maßstab der Instagramability betrachtet werden. Durch die Verschleierung der Editierung der Beiträge ver- 
schiebt sich allerdings der Gegenstand der gegenseitigen Betrachtung und das eigene (uneditierte) Leben ist konfrontiert mit den oftmals sorgfältig ausgewählten und bearbeiteten Ausschnitten der abonnierten Profile. Die Diskrepanz und der daraus entstehende Druck, das eigenen Leben dem vorgeführten „Instalife“ anzupassen, zeigen verschiedenen Studien, die zum Beispiel einen Anstieg an Essstörungen unter Jugendlichen diagnostizieren (Klapp \& Klotter 2019; Krämer, Carolin 2017). Die eigenen Daten können zwar an dieser Stelle keine Auskünfte über mögliche „offline“-Folgen geben, allerdings wird beispielsweise durch Josephine und die Verwendung des Hashtags \#formorerealtiyoninstagram dieser Konflikt auch in den Daten sichtbar.

Die Allgegenwart des Vergleichs spielt dabei zusammen mit den in das Interface eingebetteten Konkurrenzlogiken und es kristallisieren sich drei Folgen der Vergleiche heraus. (1) Schneidet man im Vergleich „gut“ ab - sei es, indem Kathi ein definierteres Sixpack als ihre Freundin hat, Sabine als inspirierender Account ausgezeichnet wird oder durch Kommentare als Vorbild bezeichnet wird, etc. -, dann mündet der Vergleich in sozialer Anerkennung. Diese Anerkennung drückt sich zwar auch durch viele Likes aus, ist allerdings nicht die einzige Quelle sozialer Anerkennung. (2) Schneidet man im Vergleich ,schlecht“ ab, ist also nicht so sportlich, dünn, energiegeladen oder positiv, steigt der Druck, sich diesem Ideal anzunähern. Praktischerweise geben einem die Profile der untersuchten Frauen oft gleich eine Anleitung an die Hand, wie man ihrem Lebensstil näherkommen kann. (3) Eine dritte Möglichkeit zeigt sich seltener in den Daten, ist aber sicherlich ebenso bedeutsam: Durch verschiedene sprachliche Akte entziehen sich die Frauen (oder in ihren Profilen markierte Personen) dem Vergleich, indem sie sich wahlweise auf ihre Einzigartigkeit berufen oder durch Aussagen wie „da kann mein Speckpack nicht mithalten“ (2020-06-13-11-45 Pos. 8) bewusst die Gleichheitsunterstellung von sich weisen.

Die Ästhetik ist dabei das entscheidende Vergleichsinstrument, auf Nachfragen nach der Bedeutung der Likes haben sowohl Josephine als auch Kathi diese zwar als wichtigen Indikator, aber nicht als entscheidende Komponente verhandelt. Vielmehr beruhen die Urteile auf subjektiven Eindrücken und dem Feedback der Follower. Die Unsicherheit über den Wert drückt sich oft durch Umfragen, direkte Bewertungsaufforderungen oder die Einforderung von Feedback aus. Da sich die Instagramability nicht objektiv messen lässt, ist sie Gegenstand von Aushandlungsprozessen. Diese Tatsache hebt die Bedeutung der Community hervor, da die Meinung der Follower entscheidend ist für die Verortung der sozialen Position und der Bewertung der 
digitalen Selbstdarstellung. Die starke Präsenz von Bewertungen ist dabei nicht selbstverständlich und liegt auch in der marktförmigen Struktur und den niederschwelligen Konkurrenzaspekten.

\section{Der Markt fragt immer nach dem Wert}

Durch das regelmäßige Posten ihrer Bilder stellen sich die Nutzer*innen kontinuierlich zur Disposition und sind dadurch zahlreich mit verschiedenen Bewertungen konfrontiert. Zum Teil nehmen die Frauen selbst die Bewertung vorweg, aber auch die Community sieht sich - gefragt oder ungefragt - dazu aufgefordert, in Nachrichten und Kommentaren eine umfassende Bewertung des oder der Dargestellten vorzunehmen. Der Akt des Postens reicht offensichtlich aus, um den Vergleich und die Bewertung zu legitimieren. Dadurch, dass die Frauen jeden Tag neue Inhalte auf der Plattform teilen, findet eine Perpetuierung der Evaluation des eigenen Körpers und des eigenen Lebens statt. Ein Bild scheint dabei mehr zu sagen als Worte, aber auch mehr als Zahlen, da die Bilder Vergleiche und Bewertungen auch für Bereiche möglich machen, die (noch) nicht technisch messbar sind. Das Bild fungiert als aussagekräftige Bestandsaufnahme und jedes Detail kann von den zahlreichen Betrachter*innen aufgegriffen oder kommentiert werden. Zwar können die Frauen durch ihr Postingverhalten beeinflussen, was Gegenstand der Beobachtung und der Bewertung ist, die Follower sind aber nicht vollständig daran gebunden. Kathi fordert die Meinung über eine Lebenseinstellung ein, die Follower allerdings reagieren vor allem mit Kommentaren zu ihrem makellosen Körper oder ihrer Kleidung.

Es liegt nahe, dass auch - wenn nicht ausschließlich - über die Anzahl der Likes Vergleiche auf verschiedenen Ebenen vollzogen werden. Die von Sascha Oswald durchgeführten Interviews zeigen ebenfalls, dass Likes nur als Indikator und nicht als zuverlässige Messgröße verhandelt werden. Das folgende längere Zitat verdeutlicht dies eindrücklich:

Aufgrund der semantischen Offenheit der Zahlen ergibt sich für viele - analog zur Unsicherheit bezüglich des Publikumsbegehrens - auch ein Deutungs- oder Interpretationsproblem. Was bedeutet ein Like eigentlich, was bedeuten so und so viele Follower? Das kann zu teils recht komplizierten Reflexionen führen, wie etwa in der folgenden Passage:

M22: „Wenn man jetzt viele Abonnenten hat und WENIG Likes, ist es glaube ich schwieriger als wenn man jetzt weniger Likes hat, aber dafür trotzdem- wenn man jetzt zehn Abonnenten hat und drei liken das, dann ist schon $\mathrm{n}$ gewisser Anteil da. Aber wenn man jetzt was weiß ich über 100 Abonnenten hat, und dann auch nur ZEHN liken. Das ist ja dann schon $\mathrm{n}$ anderes Verhältnis. Also man muss das immer denke ich auch immer ins Verhältnis mit den Abonnenten setzen und ja, wie das GEFÜHL dabei ist.“ 
M22 versucht hier, Likes und Follower ins Verhältnis zu setzen und daraus Wertigkeit abzuleiten. Letzten Endes gesteht er aber eine gewisse Unzulänglichkeit solcher scheinbar objektiven und rationalen Wertmaßstäbe ein und landet beim Gefühl als eigentlicher Autorität und Letztinstanz der Wertzuschreibung. Diese Aussage zeigt beispielhaft, wie schwierig es sich für viele Nutzende gestaltet, aus den scheinbar eindeutigen Zahlen und den daran anknüpfenden Vergleichspraktiken sinnhafte Bedeutung zu extrahieren. (Oswald $2019,10)$

Allgemein sind die Vergleichspraktiken sehr heterogen: so sind zeitliche Vergleiche ebenso üblich wie Vergleiche mit anderen User*innen oder Vergleiche des eigenen Aussehens mit einem weit verbreiteten Schönheitsideal. Die scheinbar universelle Vergleichbarkeit und ihr öffentlicher Charakter setzt die User*innen damit einer ständigen Fremd- und Eigenbeobachtung aus. Einige Tage nicht auf Instagram aktiv zu sein, wird mit dem Verlust von Followern sanktioniert und scheint nur möglich zu sein, wenn die Abwesenheit vorher angekündigt wird und der Grund dafür mit der Community geteilt wird. ${ }^{54}$ Demnach deutet alles darauf hin, dass die Selbstdarstellung und der stetige, davon nie losgelöste Vergleich niemals zur Ruhe kommen dürfen.

\footnotetext{
${ }^{54}$ Die gesichteten Ratgeber zur erfolgreichen Instagram-Nutzung betonen an mehreren Stellen die Notwendigkeit, regelmäßig zu posten. Die eigenen Daten können zwar keinen Aufschluss darüber geben, ob das Ausbleiben neuer Posts tatsächlich zur Followerverlust führt, allerdings rahmen die Frauen stets längere Pausen und erklären den Followern den Grund für ihre Abwesenheit.
} 


\section{Dynamische Selbstthematisierung}

Welche Schlussfolgerungen lassen sich nun in der vergleichenden Darstellung von digitaler Selbstvermessung und Selbstdarstellung ziehen? Worin besteht der kategoriale Unterschied quantitativer und qualitativer Selbstthematisierung? Sowohl Self-Tracking als auch Selbstdarstellung auf Instagram wurden als Orte des Vergleichens und Bewertens gerahmt, die sich durch ihren Fokus auf das Visuelle beziehungsweise die Zahl voneinander unterscheiden. Aber was bedeutet das für die Subjekte und welche Folgen ergeben sich daraus?

Vorangestellt sei noch einmal die Gleichheitsunterstellung, die hier vorgenommen wird, also unter welcher Prämisse Self-Tracking und Instagram miteinander verglichen werden. Bei beidem handelt es sich um Technologien, die eine technisch vermittelt Selbstthematisierung erlauben und damit verbunden das eigene Leben in Daten übersetzen. Beide Fälle können also als Lifelogging nach Selke definiert werden. Darin sind zunächst alle Modalitäten eingeschlossen: Text, Bild, Zahl, Ton, Video, Graphen, etc. In der bestehenden Forschung wird dabei angenommen, dass es einen Unterschied macht, wie man etwas kommuniziert, bzw. in diesem Fall, durch welche Art und Weise Aspekte des Lebens in Daten übersetzt werden. Von dieser Vorannahme ausgehend können nun quantitative und qualitative Formen der Selbstthematisierung kontrastierend betrachtet werden.

Sowohl Forschungsergebnisse aus dem Bereich des Self-Tracking als auch die eigenen empirischen Beobachtungen zeigen eine Ausdehnung von Vergleichs- und Bewertungspraktiken. Immer mehr Bereiche, auch der subjektiven und sozialen Welt, werden vergleichbar gemacht. Auch was sich ursprünglich bestimmten Vergleichskriterien entzog und als inkommensurabel und einzigartig galt, wird zunehmend durch Kennzahlen und Hashstags in Kategorien eingeteilt. Auch wenn Vergleiche kein neues soziales Phänomen darstellen, erhalten sie durch ihre ubiquitäre Verbreitung eine neue Qualität. Durch disruptive Technologien werden Vergleiche zunehmend öffentlich kommuniziert und mehr Bereichen zugänglich gemacht. Eingeschrieben in das Interface oder die Software ist darüber hinaus oft ein bestimmtes Interpretationsangebot über die Bewertung des verglichenen Gegenstands.

Mittelwerte, Graphen und Kurven, aber auch bestimmte qualitative Vorgaben einer instagramabilen Ästhetik erzeugen dabei ein Spannungsverhältnis zwischen Konformitäts- und Normalisierungsdynamiken und dem Anspruch an Einzigartigkeit. Während bei der Selbstvermessung eine Orientierung an Durchschnittswerten und Entwicklungskurven stattfindet, geben auf Ins- 
tagram die gängigen Schönheitsideale und das perfekte „Instalife“ idealtypische Lebensentwürfe vor, denen es nachzueifern gilt. Innerhalb dieses Rahmens spielen allerdings Einzigartigkeit und ein Abheben von der Masse eine wichtige Rolle. Vor allem die Übersetzung des Lebens in Zahlen geht daher einher mit einem gefühlten Individualitätsverlust, welcher aber auch für Instagram-User*innen eine relevante Rolle spielen kann. Beispielsweise dann, wenn die eigene Selbstdarstellung immer neue Elemente und Eyecatcher integrieren muss, um sich von anderen zu unterscheiden. Nicht nur der omnipräsente Vergleich unter rationalen Gesichtspunkten führt also zu einem Individualitätsverlust der Subjekte, auch der wechselseitige Vergleich unter ästhetischen Gesichtspunkten führt in gewisser Weise zur paradoxen Uniformierung unter dem Label von Einzigartigkeit und Authentizität. Durch die Strategisierung von Selbstdarstellungspraktiken auf Instagram wird das eigene Erscheinungsbild optimal inszeniert und der Community zur Disposition gestellt. Als Folge nähern sich Menschen in beiden Fällen kommensurablen Gütern an, deren Wert scheinbar über standardisierte Kriterien ermittelt werden kann. Die dabei angelegten Kriterien können dabei „hart“ im Sinne quantitativer Messgrößen oder ,weich“ im Sinne ästhetischer (und damit auf den ersten Blick schwer zu erfassenden) Maßstäben sein.

Die gesteigerten Anforderungen an die Subjekte und die Narrative des Unternehmerischen Selbst lassen sich auch in beiden Bereichen finden. Während die Fitness-Apps versprechen, die Grenzen des eigenen Körpers auszuweiten und die Subjekte noch leistungsfähiger und noch effizienter zu machen, bietet Instagram eine breite Palette an Editierungsmöglichkeiten. Diese lassen wahrgenommene Mängel verschwinden und helfen, das alltägliche Leben und die darin produzierten Bilder aufzuwerten und einen perpetuierten perfekten Look zu ermöglichen, der im „echten“ Leben nur schwer zu erreichen scheint. Darüber hinaus ermöglicht Instagram ein gewisses $\mathrm{Ma}$ an Kontrolle über das eigene impression management, da das, was für andere sichtbar ist, gezielt gepostet werden kann und andere Bilder ungesehen aussortiert werden können. Auch die damit verbundene Abweichungssensibilität und die Vorstellung des Menschen als fehlerhaft spielen hierbei eine zentrale Rolle. Während die Zahlen jede kleine Abweichung und jede Differenz diskret abbilden, führt die auf Dauer gestellte öffentliche Präsentation dazu, dass die Aufmerksamkeit für Details und mögliche Fehler steigt. In den Daten äußert sich diese awareness durch Kommentare der User*innen oder sprachliche Markierung nicht als vollkommen perfekt eingestufter Bilder und Videos. In beiden Fällen scheint das Selbst dabei nicht mehr zu ,genügen“. Während durch Selbstvermessung versucht wird, ein epistemisches Selbst (Noji \& Vormbusch 2018) hervorzubringen, das die eigenen Grenzen durch Selbsterforschung 
und -erkenntnis erweitert und sich fortlaufend optimiert, entsteht auf Instagram ein editiertes Selbst, dass sich maskiert und seine eigene Unvollkommenheit durch technische Hilfsmittel verschleiert.

Leistungs- und Steigerungsimperative einen die qualitative und quantitative Selbstthematisierung ebenso wie eine gesteigerte horizontale Kontrolle und eine immer feinere Mikrophysik der Macht. Allerdings scheint Instagram mehr Spielraum für Widerständigkeit und alternative Deutungsmuster zu lassen. Die Kombination aus Bild und Text eröffnet die Möglichkeit, Aufmerksamkeit zu generieren und diese für verschiedene Ziele, auch abseits von Leistung und Optimierung, zu nutzen. Die Analyse hat gezeigt, dass Instagram durchaus auch dazu genutzt wird, dem sich optimierenden Selbst eine Alternative entgegenzusetzen. Damit verbunden ist allerdings, dass die Selbstverortung nicht automatisiert durch die Zahlen und die Auswertung der Messdaten erfolgt, sondern interaktiv ausgehandelt werden muss. Während den Zahlen die Eigenschaft anhaftet, ,unverfälscht“ zu sein, muss die Authentizität auf Instagram aktiv hergestellt werden.

\section{Die Magie der Zahlen und Bilder}

Die Ausbreitung von Self-Tracking-Praktiken veranlasst Soziolog*innen zu der These, es entstehe eine „Herrschaft der Zahl“ (Vormbusch 2012). Diese ist allerdings nur haltbar, wenn Selbstvermessung sich als im strengen Sinne quantitative Praktik beschreiben lässt. Bettina Heintz zeigt am Beispiel der Ranglisten, dass zahlenförmige Formate zwar meist als solche (d.h. quantitativ) angesehen werden, viele allerdings nur Zahlenförmigkeit suggerieren und sich aber bei näherer Betrachtung nur ein kleiner Teil der Ranglisten als im strengen Sinne quantitativ entpuppt (vgl. Heintz 2019).

So arbeitet sie durch die Unterscheidung von Zahlen und Ziffern, Meinen und Messen und der Differenzierung zwischen ordinalen und metrischen Daten heraus, dass nicht die Zahl an sich, sondern vielmehr der Glaube an sie entscheidend ist. So geben zwar beispielsweise Bewertungssysteme in Form von Sternen, Punkten oder anderen Symbolen eine Zahlenförmigkeit vor, bilden aber keine numerischen Größen ab. Vielmehr handelt es sich dabei um Ziffern, also Zahlzeichen, mit denen ebenso wenig gerechnet werden kann wie mit Hausnummern. Ob man einen oder fünf Sterne erhält, erscheint unter dem Label einer quantitativen Bewertungsform, deren empirische Grundlage muss aber nicht notwendigerweise quantitativer Natur sein. Vielmehr kann die Einordnung in ein solches System durch subjektive und qualitative Urteile ent- 
standen sein, wie beispielsweise auf Restaurantbewertungsplattformen, aber auch in den Fitness-Apps. Auch die Likes auf Instagram beruhen auf ästhetischen und subjektiven Urteilen anderer User*innen und sind also keine Messungen, sondern Meinungen. Obwohl in der SelfTracking-Forschung gemessene Laufzeiten und eingetragene Gefühlszustände nicht analytisch getrennt betrachtet werden, lohnt es sich, zwischen diesen beiden Formen zu unterscheiden, da dadurch deutlich wird, dass Zahlen als Kommunikationsmedium dazu in der Lage sind, Aussagen mit Objektivität zu versehen, ohne dabei notwendigerweise im strengen Sinne quantitativ zu sein (vgl. ebd.). Die Forschung von Sascha Oswald zeigt darüber hinaus, dass die Likes von den Nutzer*innen durchaus kritisch verhandelt werden und die Aussagekraft der Zahlen hinterfragt wird (vgl. Oswald 2019).

Diese Überlegungen zeigen, dass die Zahlenförmigkeit als solche kein genaues Kriterium darstellt und die Unterscheidung in quantitative und qualitative Selbstthematisierungspraktiken unterkomplex ist, da diese dichotome Unterscheidung nicht offen ist für Kontinuitäten, Überlappungen und Mischformen, die sich in den Daten zeigt. Die „Herrschaft der Zahl“ kann durch die durchgeführte Untersuchung nicht verifiziert werden, da das quantitative Feedback auf Instagram nur eine von mehreren bedeutenden Bewertungspraktiken abbildet und die Zahlenförmigkeit auf subjektiven Urteilen beruht. Um diese Unterkomplexität zu überwinden, wendet Bettina Heintz das Konzept der fraktalen Unterscheidung an. Mit einer fraktalen Unterscheidung wird eine dichotome Unterscheidung in sich wiederholt und auf diese Weise eine in sich verschachtelte Hierarchie erzeugt (vgl. Heintz 2019, 66). ${ }^{55}$

\footnotetext{
${ }^{55}$ Ein klassisches Beispiel wäre Talcott Parsons AGIL-Schema. Bettina Heintz bezieht sich bei diesem Konzept auf Andrew Abbott (Abbott 2007).
} 
Angewendet auf Formen der Selbstthematisierung lässt sich durch die Anwendung einer fraktalen Unterscheidung ein ähnliches Bild erkennen:

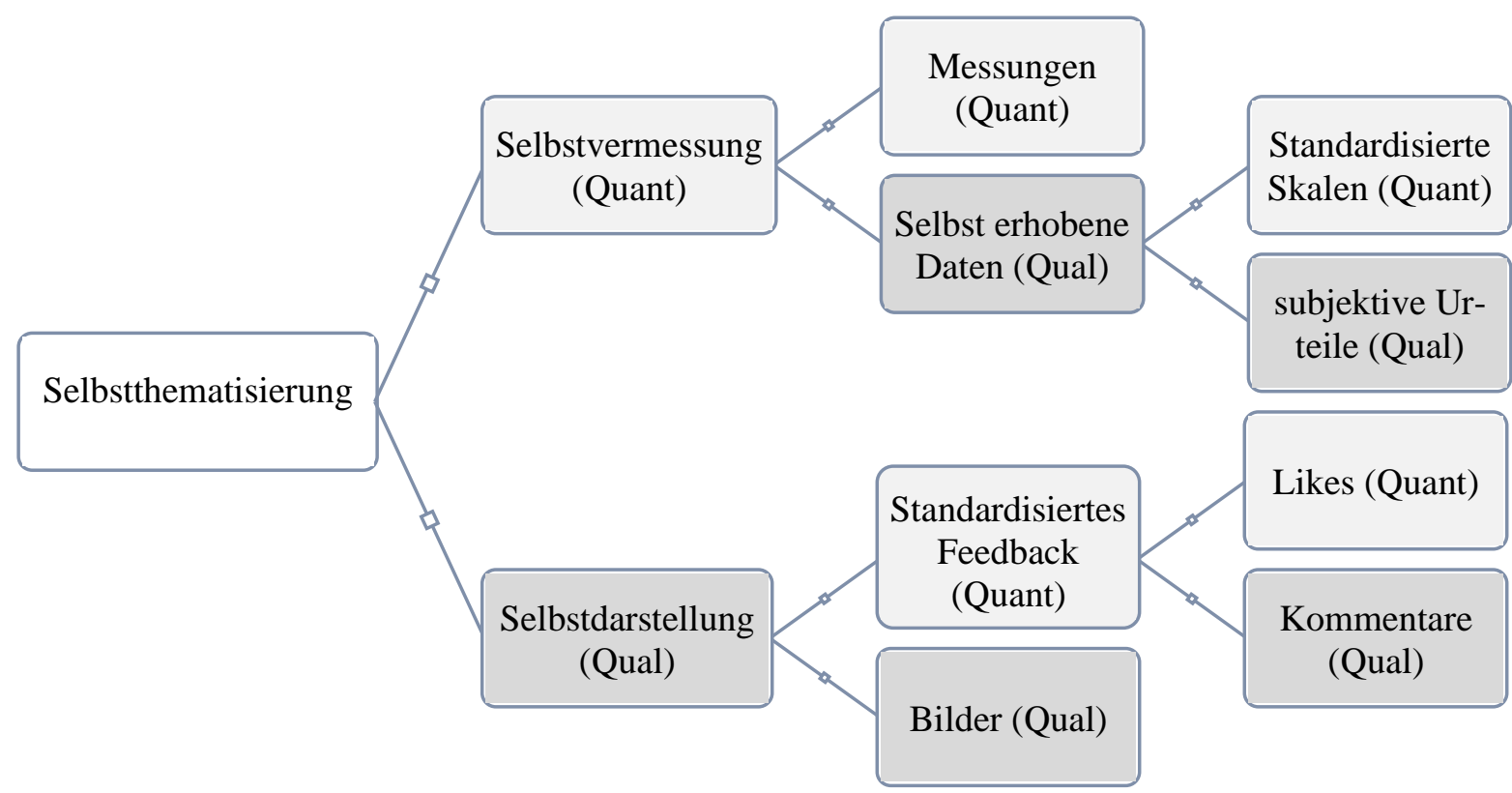

Abbildung 29: Fraktale Unterscheidung Selbstthematisierung (eigene Darstellung)

Für Bettina Heintz ergeben sich daraus verschiedene Folgen, beispielsweise die stärkere Berücksichtigung des Vertrauens in die Zahl, anstelle der reinen Betrachtung von Quantifizierung. Dabei kommt sie zu folgendem Schluss:

„Der harte These einer zunehmenden ,Herrschaft der Zahl` wäre zumindest für die Welt der Ranglisten die weichere Annahme entgegenzuhalten, dass nicht allein die Quantifizierung selbst zugenommen hat, sondern vor allem der Glaube und das Vertrauen in Zahlen - und sich die Ranglistenkonstrukteure diesen Umstand zunutze machen. Dies legt die Vermutung nahe, dass zwischen der Quantifizierungsfassade von Ranglisten und ihrem Eindringen in die Sphäre des Expressiven und Ästhetischen ein Zusammenhang besteht. Das numerische Format fungiert als eine Art ,Tarnung', um den Vergleich des Unvergleichbaren unsichtbar zu machen.“(ebd., 69)

Diese These verweist auf die Bedeutung, die der Darstellungs- bzw. Kommunikationsformzukommt und die die Resonanz entscheidend beeinflusst. Die Form der Rangliste legt eine bestimmte Rezeptions- und Benutzungsweise nahe. Die Affordanz der Technik ist ein wesentlicher Grund dafür, weshalb Vergleiche die Welt nicht einfach darstellen, sondern sie durch ihre Darstellungsweise verändern (vgl. ebd.). In den Daten äußert sich die Bedeutung der Affordanz 
durch die niederschwellige Konstruktion von Konkurrenz zwischen Mitgliedern der Community und die unterschiedliche Nutzung der qualitativen und quantitativen Bewertungsinstrumente. Auch wenn die Aussagefähigkeit der Likes von Nutzer*innen kritisch hinterfragt wird oder ihre Bedeutung nivelliert wird, sind die quantitativen Likes wichtig für die Konstruktion der Instagramability und des „Instalife“.

Ähnlich wie durch die Selbstvermessung kann das tägliche Feedback auf Instagram als Anpassungsmotor dienen. Allerdings kommt Sascha Oswald zu dem Ergebnis, dass in den von ihm durchgeführten Interviews der Like als objektiver und rationaler Wertmaßstab als eher unzulänglich empfunden wird und vielmehr das Gefühl als entscheidendes Kriterium der Wertzuschreibung dient (vgl. Oswald 2019, 10). Zudem kann die Wertigkeit und Position im Feld einerseits ausgezählt, das heißt quantitativ bestimmt werden (über Likes und Follower), die Zahlen selbst aber orientieren sich an qualitativen, abstrakten und kaum greifbaren ästhetischen Werten (beispielsweise einem kollektiven Stil, der implizit gewusst wird, wechselnden Trends usw.) (vgl. ebd., 11).

Die eigenen Daten zeigen, dass die Herrschaft der Zahl durchaus kritisch gesehen werden muss und dass die Fokussierung Stefan Selkes auf quantitative Lifelogs fälschlicherweise die Quantifizierung als Auslöser für gestiegene Abweichungssensibilität und Steigerungszwänge benennt. Die Analyse von digitaler Selbstdarstellung auf Instagram zeigt, dass nicht notwendigerweise Zahlen notwendig sind, um die Subjekte unter Steigerungs- und Leistungsimperative zu unterwerfen, sondern dass vielmehr der öffentliche und auf Dauer gestellte Vergleich entscheidend ist. Dem Glauben an die Zahl kann an dieser Stellte der Glaube an das Bild hinzugefügt werden, da auch auf Instagram gepostete Bilder von den User*innen als valides Abbild des Selbst und des eigenen Lebens zur Selbstthematisierung genutzt werden, ungeachtet der teilweise umfassenden Inszenierung und Bearbeitung der Bilder. Aufbauend auf die in Kapitel 2.2 verhandelte Attraktivität numerischer Vergleiche können zwar quantitative und qualitative Vergleiche nicht gleichgesetzt werden, allerdings zeigt sich, dass die Bedeutung der Quantifizierung in der Forschung zur digitalen Selbstvermessung überschätzt wird. Die Kontrastierung von qualitativer Selbstdarstellung und quantitativer Selbstvermessung untermauert die These, dass die Art und Weise der Kommunikation eine Rolle spielt, jedoch kann die Quantifizierung an sich, wie auch Bettina Heintz für Ranglisten herausarbeitet, nicht als entscheidender Faktor bei der Zunahme von Vergleichs- und Bewertungspraktiken herangezogen werden. 


\section{Fazit}

Eingangs wurde die Frage gestellt, worin der kategoriale Unterschied zwischen quantitativer und qualitativer Selbstthematisierung besteht. Durch die vergleichende Betrachtung hat sich gezeigt, dass die Zahlenförmigkeit nicht unbedingt ein trennscharfes Kriterium darstellt, auch wenn die Forschung zu Selbstvermessung dies nahelegen und die Steigerungsdynamiken und sich ausdehnende Ökonomisierung auf die zunehmende Quantifizierung zurückführen würde. So wird auf Instagram zwar ein klarer Fokus auf visuelle Ästhetik gelegt, durch Likes und Followerzahlen aber eine quantitative Komponente eingeführt und auch im Bereich der digitalen Selbstvermessung ist die quantitative Vermessung nicht isoliert, sondern wird bspw. durch manuelle Eingaben und Einschätzungen ergänzt. Die Unterscheidung zwischen qualitativer und quantitativer Selbstthematisierung ist also zum einen vor allem eine analytische Unterscheidung, die sich in der Alltagswelt der Menschen nicht diskret feststellen lässt. Zum anderen hat die Analyse digitaler Selbstdarstellung gezeigt, dass sich auf Instagram ähnliche Mechanismen der Anpassung und Optimierung finden lassen, wie sie beispielsweise Stefan Selke beschreibt. Die vergleichende Betrachtung zeigt also, dass es schwer ist einen kategorialen Unterschied zwischen digitaler Selbstvermessung und -darstellung zu benennen. Durch die Anwendung der fraktalen Unterscheidungen werden Überschneidungen sichtbar die die Herrschaft der Zahl zumindest ein Stück weit ins Wanken bringen.

Die Arbeit verfolgte das Ziel, Stefan Selkes Definition von Lifelogging ernst zu nehmen und die Übersetzung des Lebens in Daten über die quantitative Vermessung des Körpers und der Gefühle hinaus zu erweitern. In beiden betrachteten Fällen - der quantitativen digitalen Selbstvermessung sowie der qualitativen digitalen Selbstdarstellung - beeinflussen die Technologie und der kulturelle Wandel die Subjekte, auf der Ebene der Subjektformierung und der Subjektivierungsweisen. Die Beschaffenheit der Technologie ebenso wie die in sie eingeschrieben und durch sie reproduzierten sozialen Werte und Normen wirken auf die Menschen ein und adressieren sie als formbare Subjekte. Instagram und Self-Tracking - beide spiegeln also Facetten eines formbaren Subjekts wider, das zwar jeweils unterschiedliche Eigenschaften aufweist, dem aber die gleichen Dynamiken zugrunde liegen. Die Subjekte, so wie sie sind, „genügen“ angesichts der stetig steigenden Anforderungen scheinbar nicht mehr und werden deshalb wahlweise maskiert oder demaskiert, um wettbewerbsfähig zu bleiben.

Die technischen Geräte rücken näher an den Körper heran und erschließen mehr Bereiche der sozialen und subjektiven Welt. Dabei beeinflussen neue soziale Taxonomien wie NikeFuels 
oder die Instagramability den Blick auf die Welt. Durch die regelmäßige Übersetzung bedeutsamer Aspekte des Lebens in Daten und dem öffentlichen Teilen dieser, setzen sich die Menschen einer ständigen (Selbst- und Fremd-) Beobachtung aus, wodurch sich Vergleichspraktiken ausdehnen. Während auf Instagram die aus den Vergleichen abgeleitete Bewertung prinzipiell offener ist und ein höheres Widerständigkeitspotenzial aufweist, legen Self-TrackingApps eine stärkere normative Wertung und eine bestimmte Interpretation der dort erhobenen Daten nahe. In Bezug auf den kulturellen Wandel bestätigen sich in beiden Bereiche viele Aspekte der von Hartmut Rosa beschriebenen dynamischen Stabilisierung, in der Steigerungsimperative zum Strukturprinzip werden. Anders als es allerdings Rosa vermuten lässt, ist nicht die Zahl das ausschlaggebende Kriterium, sondern der öffentliche Vergleich und allgemein die zunehmende Vergleichbarmachung der subjektiven und sozialen Welt, sei es in Form von Messdaten oder geteilten Bildern.

Sowohl Zahlen als auch Bilder gehen einher mit dem Versprechen einer unverfälschten Wiedergabe der Realität, auch wenn diese Unverfälschtheit relativ zu betrachten ist. Weder die objektive Messung noch fotografische Darstellung können dieses Versprechen einlösen und verschiedene Mechanismen tragen zur Verschleierung dieser Abweichung zwischen Wirklichkeit und Übersetzung bei. Dadurch tritt vor allem der Glaube an die Zahl bzw. das Bild in den Fokus. Den entscheidenden Aspekt bei der technisch vermittelten Selbstvermessung bzw. -darstellung bildet damit nicht die Objektivitätsfiktion der Zahlen, oder die Verschleierung der Inszenierung an sich. Entscheidend ist vielmehr das Vertrauen in diese beiden Vergleichsinstrumente. $\mathrm{Ob}$ mithilfe von Zahlen oder Bildern kommuniziert wird, kann eine untergeordnete Rolle spielen, solang die gewählte Form als valides Instrument für Vergleiche und Bewertungen angesehen wird und damit die in den Vergleichen produzierten Aspekte der Konkurrenz und Optimierung legitimiert werden können. Auch die von Bettina Heintz untersuchten Ranglisten sind nicht primär quantitative Instrumente, sondern in erster Linie Vergleichsinstrumente, die quantitativ sein und Konkurrenz initiieren können, aber nicht müssen. Nicht Quantifizierung ist damit das Besondere, sondern ihre Vergleichbarmachung und die Ausdehnung auf Bereiche, die sich bislang einer komparativen Optik entzogen haben (vgl. Heintz 2019, 70). Eben diese Ausdehnung des Vergleichshorizonts lässt sich sowohl in (quantitativen) Selbstvermessungs- als auch (qualitativen) Selbstdarstellungspraktiken beobachten.

Abschließend hilft es, sich die Frage zu stellen, auf welche Probleme die untersuchten Vergleichs- und Bewertungspraktiken reagieren: Zum einen können standardisierte Vergleichsinstrumente helfen, der Pluralität der Moderne entgegenzuwirken und durch ihren reduzierenden, 
vereinfachenden Charakter eine Entlastungsfunktion erfüllen. Aspekte, die noch als einzigartig und unvergleichlich angesehen werden, werden zunehmend in die Welt des Kommensurablen überführt. Der sich daraus ergebende Widerspruch zwischen Einzigartigkeit und Einheitlichkeit wird dabei durch die Invisibilisierung des Vergleichs aufgelöst oder zumindest verschleiert (vgl. ebd., 71). Die Regelmäßigkeit des Lifeloggings, sei es durch tägliches Posten oder Schritte Zählen, wirkt der zeitlichen Perspektive der dynamischen Stabilisierung entgegen, da es dadurch möglich wird, den Erfolg immer wieder aufs Neue sichtbar zu machen und so den eigenen Status zu sichern. Zuletzt deutet alles darauf hin, dass die untersuchten Vergleichspraktiken ihre Wirkung vor allem dann entfalten, wenn sie öffentlich kommuniziert werden (vgl. ebd.). Durch den ständigen und eben auch öffentlichen Vergleich und die damit verbundene potenzielle Fremdbeobachtung funktionieren sie weitaus subtiler als das idealtypische Panopticon, da schließlich der Wärter fast vollständig in die Subjekte verlagert wird.

Weitere Untersuchungen zu diesem Thema könnten im Anschluss daran stärker die Legitimierung von verschiedenen Vergleichsinstrumenten in den Blick nehmen. Durch die Beschaffenheit des Forschungsdesgins ist es nur möglich, gepostete Bilder und Videos zu analysieren -der „Blick hinter die Fassade“ bleibt verschlossen. Deshalb wäre es spannend, in zukünftigen empirischen Untersuchungen die Art und Weise, wie Beiträge aufgenommen, editiert und selektiert werden, zu analysieren. Diese Vorgehensweise könnte an die Untersuchung des Projekts „Das vermessene Leben“ anschließen, in dem im Jenaer Teilprojekt bereits Legitimationsstrategien durch Zahlen in der öffentlichen Verwaltung analysiert werden. Auch erheben die hier erhobenen Daten keinen Anspruch auf Repräsentativität. Sowohl die Nutzungstypen als auch die Postingfrequenz ist sehr heterogen und die Arbeit beschränkt sich auf Frauen, die sehr häufig Bilder auf der Plattform teilen. Nicht berücksichtigt sind also hier Menschen, die sich vor allem passiv auf Instagram bewegen. Da sich gezeigt hat, dass auch auf Instagram Zahlen relevante Bezugsgrößen für Vergleiche und Bewertungen bilden, kann es sich in zukünftigen Untersuchungen lohnen, zwar die Quantifizierung nicht aus dem Blick zu verlieren, aber dennoch auch den Blick auszuweiten und digitale Bewertungspraktiken und ihre Folgen für die Subjekte allgemein mitzuberücksichtigen. Denn insgesamt konnte mit dieser Arbeit gezeigt werden, dass neue disruptive Technologien im Zusammenhang mit dem kulturellen Wandel nicht nur eine Herrschaft der Zahl hervorbringen, sondern diese durch eine Herrschaft des Bildes zumindest ergänzt werden muss. 


\section{Literaturverzeichnis}

Abbott, Andrew D. 2007. Chaos of Disciplines. [Nachdr.]. Chicago, Ill. Univ. of Chicago Press.

Abidin, Crystal. 2016. "Visibility labour: Engaging with Influencers' fashion brands and \#OOTD advertorial campaigns on Instagram." Media International Australia 161 (1): 86100. doi:10.1177/1329878X16665177.

Araújo, Camila, Raquel Prates, Ana Paula Couto da Silva, und Wagner Jr. Meira (Hrsg.). 2014. Its Not Just a Picture: Revealing Somer User Practices in Instagram. Conference Paper.

Arlinghaus, Franz-Josef, Walter Erhart, und Lena Gumpert (Hrsg.). 2020. Sich selbst vergleichen: Zur Relationalität autobiographischen Schreibens vom 12. Jahrhundert bis zur Gegenwart. Histoire.

Bakhshi, Saeideh, David A. Shamma, und Eric Gilbert. 2014. "Faces engage us." CHI, no. 1: 965-74. doi:10.1145/2556288.2557403.

Barlösius, Eva. 2014. Dicksein: Wenn der Körper das Verhältnis zur Gesellschaft bestimmt. Frankfurt am Main: Campus-Verlag.

Bartel, Stefanie. 2014. Farben im Webdesign: Symbolik, Farbpsychologie, Gestaltung. Softcover reprint of the original 1st ed. 2003. X.media.press. Berlin: Springer.

Bauman, Zygmunt, und Frank Jakubzik. 2007. Leben in der flüchtigen Moderne. Orig.-Ausg., 1. Aufl. IWM-Vorlesungen zu den Wissenschaften vom Menschen 2503. Frankfurt am Main: Suhrkamp.

Becker, Frank, Angelika Epple, Walter Erhart, Susan S. Friedman, Johannes Grave, Thomas Hippler, Barbara Korte, Helmut Peitsch, Rajagopala Radhakrishan, und Daniel S. Chassé (Hrsg.). 2015. Die Welt beobachten: Praktiken des Vergleichens. 1st ed. Frankfurt am Main: Campus Verlag.

Beckmann, Susanne C., und Roy Langer. 2009. "Netnographie." In Qualitative Marktforschung: Konzepte - Methoden - Analysen, Herausgegeben von Renate Buber and Hartmut H. Holzmüller. 2., überarbeitete Auflage, 221-26. Lehrbuch. Wiesbaden: Gabler Verlag / GWV Fachverlage GmbH Wiesbaden.

Beuscart, Jean-Samuel, und Kevin Mellet. 2017. "Die Online-Stimmen von Verbrauchern in Form bringen: Algorithmischer Apparat oder Bewertungskultur?” In Algorithmuskulturen: Über die rechnerische Konstruktion der Wirklichkeit, Herausgegeben von Robert Seyfert and Jonathan Roberge, 107-31. Kulturen der Gesellschaft 26. Bielefeld: transcript Verlag.

Boellstorff, Tom, Bonnie Nardi, Celia Pearce, T. L. L. Taylor, T. L. Taylor, und George E. Marcus. 2012. Ethnography and Virtual Worlds: A Handbook of Method. Princeton: Princeton University Press.

Boltanski, Luc, Ève Chiapello, und Franz Schultheis. 2006. Der neue Geist des Kapitalismus. Broschierte Ausgabe. Edition discours 38. Konstanz: UVK.

Bröckling, Ulrich. 2013. "Der präventive Imperativ und die Ökonomisierung des Sozialen.” Public Health Forum 21 (81). 
Buchanan, Elizabeth A. 2019. "Ethics in Digital Research.” In Friese, Nolden, Rebane, und Schreiter 2019, 375-84.

Caldeira, Sofia P., Sander Ridder, und Sofie van Bauwel. 2018. "Exploring the Politics of Gender Representation on Instagram: Self-representations of Femininity." DiGeSt. Journal of Diversity and Gender Studies 5 (1): 23. doi:10.11116/DiGeSt.5.1.2.

— 2020. “'Everybody needs to post a selfie every once in a while': exploring the politics of Instagram curation in young women's self-representational practices." Information, Communication \& Society 30 (3): 1-18. doi:10.1080/1369118X.2020.1776371.

Castells, Manuel. 2017. Der Aufstieg der Netzwerkgesellschaft: Das Informationszeitalter. Wirtschaft. Gesellschaft. Kultur. Band 1. 2 Aufl. Neue Bibliothek der Sozialwissenschaften. Wiesbaden: Springer.

Castells, Manuel, und Reinhart Kößler. 2003. Der Aufstieg der Netzwerkgesellschaft. Durchges. Nachdr. der 1. Aufl. Das Informationszeitalter Wirtschaft, Gesellschaft, Kultur / Manuel Castells. Übers. von Reinhart Kößler ; Teil 1. Opladen: Leske + Budrich.

Davy, Ulrike, Johannes Grave, Marcus Hartner, Ralf Schneider, und Willibald Steinmetz. 2019. "Grundbegriffe für eine Theorie des Vergleichens. Ein Zwischenbericht: Working Paper des SFB 1288." no. 3. doi:10.4119/unibi/2939563.

Dörre, Klaus. 2010. “Normalarbeit und Prekarität.” Zeitschrift für Sozialreform 56 (4): 439-42. doi:10.1515/zsr-2010-0409.

Dörre, Klaus, und Hartmut Rosa. 2015. “Antrag auf Weiterförderung der Kollegforscher_innengruppe Landnahme, Beschleunigung, Aktivierung: Dynamik und (De-)Stabilisierung moderner Wachstumsgesellschaften.".

Duttweiler, Stefanie. 2018. "Daten statt Worte?! Bedeutungsproduktion in digitalen Selbstvermessungspraktiken." In Bedeutende Daten: Modelle, Verfahren und Praxis der Vermessung und Verdatung im Netz, Herausgegeben von Thorben Mämecke, Jan-Hendrik Passoth, und Josef Wehner, 251-76. Medien, Kultur, Kommunikation. Wiesbaden: Springer VS.

Duttweiler, Stefanie, Robert Gugutzer, Jan-Hendrik Passoth, und Jörg Strübing (Hrsg.). 2016. Leben nach Zahlen: Self-Tracking als Optimierungsprojekt? Digitale Gesellschaft 10. Bielefeld: transcript.

Espeland, Wendy N., und Mitchell L. Stevens. 2008. “A Sociology of Quantification.” Arch. eur. sociol. 49 (3): 401-36. doi:10.1017/S0003975609000150.

Foucault, Michel. 1966. Die Ordnung der Dinge: Eine Archäologie der Humanwissenschaften. 24. Auflage 2017. Suhrkamp-Taschenbuch Wissenschaft 96. Frankfurt am Main: Suhrkamp. 1982. The Archaeology of Knowledge. New York: Pantheon Books. 1990. The History of Sexuality. Vintage Books ed. New York: Vintage Books. 1991. Der Wille zum Wissen. 21. Auflage. Suhrkamp-Taschenbuch Wissenschaft 716. Frankfurt am Main: Suhrkamp.

1993. Überwachen und Strafen: Die Geburt des Gefängnisses. 16. Auflage. SuhrkampTaschenbuch 2271. Translated by Walter Seitter. Frankfurt am Main: Suhrkamp. 
1994. "Warum ich Macht untersuche: Die Frage des Subjekts." In Michel Foucault: Jenseits von Strukturalismus und Hermeneutik, Herausgegeben von Hubert L. Dreyfus, Paul Rabinow, und Michel Foucault. 2. Aufl., 243-50. Neue wissenschaftliche Bibliothek. Weinheim: Beltz-Athenäum.

Friese, Heidrun, Marcus Nolden, Gala Rebane, und Miriam Schreiter (Hrsg.). 2019. HANDBUCH SOZIALE PRAKTIKEN UND DIGITALE ALLTAGSWELTEN. [S.1.]: VS.

Frisch, Thomas. 2019. "Digitale Bewertungspraktiken im Tourismus 2.0: Grenzüberschreitung und Normalisierungsdruck." In Digitale Bewertungspraktiken: Für eine Bewertungssoziologie des Digitalen, Herausgegeben von Jonathan Kropf and Stefan Laser, 41-71. Soziologie des Wertens und Bewertens. Wiesbaden: Springer.

Fujimoto, Kenichi. 2005. “, Kenichi (2005): the Third Stage Paradigm: Territory Machines from the Girl's Pager Revolution. in: Ito,." In Personal, Portable, Pedestrian: Mobile Phones in Japanese Life, Herausgegeben von Daisuke Okabe, Mizuko Itō, Misa Matsuda, und Roland Asanger. 4 Aufl., 77-101. Cambridge, Mass: MIT Press; Psychologie-Verl.-Union.

Goffman, Erving. 1976. Frame Analysis: An Essay on the Organization of Experience. 3. print. Cambridge, Mass. Harvard Univ. Press.

1981. Geschlecht und Werbung. Deutsche Erstausgabe. Edition Suhrkamp $1085=$ Neue Folge, Band 85. Frankfurt am Main: Suhrkamp.

Gordon, Colin. 2009. "Govermental Rationality: an Introduction.” In The Foucault Effect: Studies in Governmentality ; with Two Lectures by and an Interview with Michel Foucault, Herausgegeben von Graham Burchell and Michel Foucault. [Nachdr.], 1-51. Chicago: Chicago Press.

Hartson, HR. 2003. "Cognitive, physical, sensory, and functional affordances in interaction design.” Behaviour \& Information Technology 22 (5): 315-18.

Heintz, Bettina. 2007. "Soziale und funktionale Differenzierung: Überlegungen zu einer Interaktionstheorie der Weltgesellschaft.” Soziale Systeme 13 (1+2): 343-56.

—. 2010. "Numerische Differenz: Überlegungen zu einer Soziologie des (quantitativen) Vergleichs.” Zeitschrift für Soziologie 39 (3). doi:10.1515/zfsoz-2010-0301.

- 2016. “„Wir leben im Zeitalter der Vergleichung.“: Perspektiven einer Soziologie des Vergleichs.” Zeitschrift für Soziologie 45 (5): 305-23. doi:10.1515/zfsoz-2015-1018.

. 2019. "Vom Komparativ zum Superlativ: Eine kleine Soziologie der Rangliste." In Nicolae, Endreß, Berli, undBischur 2019, 45-81.

Heyen, Nils B. 2019. "Von der Selbstvermessung zur Selbstexpertisierung: Zur Produktion von selbstbezogenem Wissen durch Personal Science.” In Personal Health Science: Persönliches Gesundheitswissen zwischen Selbstsorge und Bürgerforschung, Herausgegeben von Nils B. Heyen, Sascha Dickel, und Anne Brüninghaus, 23-43. Öffentliche Wissenschaft und gesellschaftlicher Wandel. Wiesbaden: Springer VS. 
Heyen, Nils B., Sascha Dickel, und Anne Brüninghaus (Hrsg.). 2019. Personal Health Science: Persönliches Gesundheitswissen zwischen Selbstsorge und Bürgerforschung. Öffentliche Wissenschaft und gesellschaftlicher Wandel. Wiesbaden: Springer VS.

Hopkins, Julian. 2019. “The Lifestyle Blog Genre.” In Friese, Nolden, Rebane, undSchreiter 2019, 130-40.

Illouz, Eva. 2012. Cold Intimacies: The Making of Emotional Capitalism. Repr. Cambridge: Polity Press.

Katz, James E., und Mark A. Aakhus (Hrsg.). 2006. Perpetual Contact: Mobile Communication, Private Talk, Public Performance. 4. print. Cambridge: Cambridge Univ. Press.

Klapp, Anna-Lena, und Johann C. Klotter. 2019. “\#Fitness vs. \#BodyPositive - die unterschiedliche soziale Konstruktion von Gesundheit auf Instagram." Prävention und Gesundheitsförderung 14 (4): 362-67. doi:10.1007/s11553-019-00708-5.

Kleemann, Frank. 2012. "Subjektivierung von Arbeit - eine Reflexion zum Stand des Diskurses.” AIS-Studien 5 (2): 6-20. doi:10.21241/ssoar.64781.

Kleemann, Frank, Uwe Krähnke, und Ingo Matuschek. 2009. Interpretative Sozialforschung: Eine praxisorientierte Einführung. 1. Aufl. Wiesbaden: VS.

Knoblauch, Hubert. 2002. "Fokussierte Ethnographie als Teil einer soziologischen Ethnographie: Zur Klärung einiger Missverständnisse.” Sozialer Sinn 1: 129-35.

Knorr-Cetina, Karin. 2012a. "Die synthetische Situation.” In Sozialität in Slow Motion: Theoretische und empirische Perspektiven; Festschrift für Jörg Bergmann, Herausgegeben von Ruth Ayaß und Christian Meyer, 82-106. Wiesbaden: Springer VS.

2012b. "Skopische Medien: Am Beispiel der Architektur von Finanzmärkten.” In Mediatisierte Welten: Forschungsfelder und Beschreibungsansätze, Herausgegeben von Friedrich Krotz und Andreas Hepp, 167-97. Medien - Kultur - Kommunikation. Wiesbaden: Springer VS (Verl. für Sozialwissenschaften).

Koskinen, Ilpo K. 2007. Mobile Multimedia in Action. New Brunswick, NJ: Transaction Publ.

Kozinets, Robert V. 1998. “On Netnography: Initial Reflections on Consumer Research Investigations of Cyberculture." NA - Advances in Consumer Research Volume (25): 366-71. https://www.acrwebsite.org/volumes/8180/volumes/v25/NA-25/full.

Kramer, Michaela, und Maria Schreiber. 2016. ""Verdammt schön": Methodologische und methodische Herausforderungen der Rekonstruktion von Bildpraktiken auf Instagram." ZGF 17 (1_2): 81-106.

Krämer, Carolin. 2017. Instagram und Körperbild: Eine quantitative Onlinebefragung zur Nutzung und Wirkung von Sport- und Fitnessinhalten auf Instagram. München: neobooks.

Krämer, Franz, und Denise Klinge. 2019. "Körper als formbare Formungsinstrumente: Zur Art und Weise der Körperthematisierung in Self-Tracking-Werbebildern im Internet." In SelfTracking, Selfies, Tinder und Co, Herausgegeben von Daniel Rode and Martin Stern, 10425. Bielefeld: transcript Verlag. 
Kropf, Jonathan, und Stefan Laser (Hrsg.). 2019. Digitale Bewertungspraktiken: Für eine Bewertungssoziologie des Digitalen. Soziologie des Wertens und Bewertens. Wiesbaden: Springer.

Krüger, Steffen, und Ane Charlotte Spilde. 2019. "Judging books by their covers - Tinder interface, usage and sociocultural implications." Information, Communication \& Society 43 (1): 1-16. doi:10.1080/1369118X.2019.1572771.

Manovich, Lev. 2017. Instagram and Contemporary Image. http://manovich.net/index.php/projects/instagram-and-contemporary-image.

Marwick, Alice E. 2015. "Instafame: Luxury Selfies in the Attention Economy." Public Culture 27 (1 75): 137-60. doi:10.1215/08992363-2798379.

Moon, Jang H., Eunji Lee, Jung-Ah Lee, Tae R. Choi, und Yongjun Sung. 2016. "The role of narcissism in self-promotion on Instagram." Personality and Individual Differences 101:2225. doi:10.1016/j.paid.2016.05.042.

Neckel, Sighard. 2005. "Emotion by design.” BJfS 15 (3): 419-30. doi:10.1007/s11609-0050208-1.

Nicolae, Stefan, Martin Endreß, Oliver Berli, und Daniel Bischur (Hrsg.). 2019. (Be)Werten. Beiträge zur sozialen Konstruktion von Wertigkeit. Soziologie des Wertens und Bewertens. Wiesbaden, Germany: Springer VS.

Nietzsche, Friedrich. 2005. Menschliches, Allzumenschliches I und II. Neuausg., 3., Aufl. Berlin: Dt. Taschenbuch Verl.

Noji, Eryk, und Uwe Vormbusch. 2018. "Kalkulative Formen der Selbstthematisierung und das epistemische Selbst.” psychosozial 41 (2): 16-34.

Oswald, Sascha. 2019. "Subjektformierungen und Evaluationsfunktionen in skopischen Medien am Beispiel der Social Networking Site Instagram: Beitrag zur Veranstaltung »Komplexe Handlungen und dynamische Öffentlichkeiten: Zum Verhältnis von Raum, Zeit und Interaktion in vernetzten Medienwelten « der Sektion Medien- und Kommunikationssoziologie." Komplexe Dynamiken globaler und lokaler Entwicklungen. Verhandlungen des 39. Kongresses der Deutschen Gesellschaft für Soziologie in Göttingen 201839.

Polanyi, Karl. 2017. The great transformation: Politische und ökonomische Ursprünge von Gesellschaften und Wirtschaftssystemen. 13. Auflage, Lizenzausgabe. Suhrkamp-Taschenbuch Wissenschaft 260. Berlin: Suhrkamp Taschenbuch Verlag.

Prainsack, Barbara. 2020. "The political economy of digital data: introduction to the special issue.” Policy Studies 97:1-8. doi:10.1080/01442872.2020.1723519.

Rosa, Hartmut. 2006. "Wettbewerb als Interaktionsmodus." Leviathan 34 (1): 82-104. doi:10.1007/s11578-006-0005-z.

2016a. Beschleunigung: Die Veränderung der Zeitstrukturen in der Moderne. 11. Auflage. Suhrkamp-Taschenbuch Wissenschaft 1760. Frankfurt am Main: Suhrkamp.

—. 2016b. Resonanz: Eine Soziologie der Weltbeziehung. 1. Aufl. Berlin: Suhrkamp. 
Rosenthal, Gabriele (Hrsg.). 2015. Interpretative Sozialforschung: Eine Einführung. 5., aktualisierte und ergänzte Auflage. Grundlagentexte Soziologie. Weinheim, Basel: Beltz Juventa.

Schaupp, Simon. 2016. "“Wir nennen es flexible Selbstkontrolle: Self-Tracking als Selbsttechnologie des kybernetsichen Kapitalismus." In Duttweiler, Gugutzer, Passoth, und Strübing 2016, 63-86.

Schütz, Alfred. 1971. Gesammelte Aufsätze I: Wissenschaftliche Interpretation und Alltagsverständnis menschlichen Handelns. Den Haag: Nijhoff.

Selke, Stefan (Hrsg.). 2016a. Lifelogging: Digitale Selbstvermessung und Lebensprotokollierung zwischen disruptiver Technologie und kulturellem Wandel. Wiesbaden: Springer VS.

. 2016b. “Quantified Self statt Hahnenkampf: Die neue Taxonomie des Sozialen.”. Bundesgesundheitsblatt, Gesundheitsforschung, Gesundheitsschutz $59 \quad$ (8): 963-69. doi:10.1007/s00103-016-2381-0.

. 2019. “Lifelogging.” In Friese, Nolden, Rebane, und Schreiter 2019, 323-34.

. 2020. "Zwischen Delirium der Rationalität und Verlust biografischer Imaginationsfähigkeit." In Datafizierung und Big Data: Ethische, anthropologische und wissenschaftstheoretische Perspektiven, Herausgegeben von Klaus Wiegerling, Michael Nerurkar, und Christian Wadephul, 229-59. Anthropologie - Technikphilosophie - Gesellschaft.

Sheldon, Pavica, und Katherine Bryant. 2016. "Instagram: Motives for its use and relationship to narcissism and contextual age." Computers in Human Behavior 58:89-97. doi:10.1016/j.chb.2015.12.059.

Stanfill, Mel. 2015. "The interface as discourse: The production of norms through web design." New Media \& Society 17 (7): 1059-74. doi:10.1177/1461444814520873.

Stark, Christopher. 2016. "Der neoliberale Zeitgeist als Närboden für die digitale Selbstvermessung: Selbstevaluation - allumfassend, 86.400 Sekunden am Tag, 365 Tage im Jahr.” In Selke 2016a, 287-308.

Unger, Hella von, Petra Narimani, und Rosaline M’Bayo (Hrsg.). 2014. Forschungsethik in der qualitativen Forschung: Reflexivität, Perspektiven, Positionen. Wiesbaden: Springer VS.

Vaterlaus, Mitchell J., Kathryn Barnett, Cesia Roche, und Jimmy A. Young. 2016. ""Snapchat is more personal": An exploratory study on Snapchat behaviors and young adult interpersonal relationship." Computers in Human Behavior (62): 594-601.

Virilio, Paul. 2015. Rasender Stillstand: Essay. Ungek. Ausg., 5. Aufl. Fischer-Taschenbücher Kultur \& Medien 13414. Frankfurt am Main: Fischer-Taschenbuch-Verl.

Vormbusch, Uwe. 2004. “Accounting. Die Macht der Zahlen im gegenwärtigen Kapitalismus.” BJfS 14 (1): 33-50. doi:10.1007/BF03204695.

- 2012. Die Herrschaft der Zahlen: Zur Kalkulation des Sozialen in der kapitalistischen Moderne. Frankfurter Beiträge zur Soziologie und Sozialphilosophie 15. Frankfurt am Main, New York: Campus Verlag. Habilitationsschrift.

. 2019. "Soziokalkulation: Zur Ausweitung des Bewertungshorizonts in der Gegenwartsgesellschaft.” In Nicolae, Endreß, Berli, und Bischur 2019, 23-45. 
Vormbusch, Uwe, und Karolin Kappler. 2016. "Leibschreiben: Zur medialen Repräsentation des Körperleibes im Feld der Selbstvermessung.” In Selke 2016a, 207-31.

Weber, Max. 2018. Verstehende Soziologie und Werturteilsfreiheit: Schriften und Reden 19081917. Gesamtausgabe Schriften und Reden Band 12. Tübingen: J.C.B. Mohr (Paul Siebeck).

Wiedemann, Lisa. 2016. "Datensätze der Selbstbeobachtung: Daten verkörpern und Leib vergessen?" In Selke 2016a, 65-90.

Zillien, Nicole, Gerrit Fröhlich, und Mareike Dötsch. 2015. “Zahlenkörper.” In Präsenzen 2.0: Körperinszenierung in Medienkulturen, Herausgegeben von Kornelia Hahn and Martin Stempfhuber, 77-94. Medienkulturen im digitalen Zeitalter. Wiesbaden: Springer VS.

Zillien, Nicole, Daniel Kofahl, und Gerrit Fröhlich. 2016. "Ernährungsbezogene Selbstvermessung. Von der Diätetik bis zum Diet Tracking." In Duttweiler, Gugutzer, Passoth, und Strübing 2016, 123-40.

Zuboff, Shoshana. 2018. Das Zeitalter des Überwachungskapitalismus. Frankfurt, New York: Campus Verlag. 


\section{Abbildungsverzeichnis}

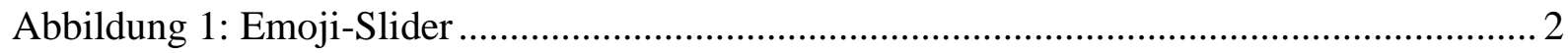

Abbildung 2: Startbildschirm Instagram (Screenshot erstellt am 18.09.2020) ....................... 44

Abbildung 3: Lokalisten (links) und TikTok (rechts); Screenshot erstellt am 18.09.2020) .... 45

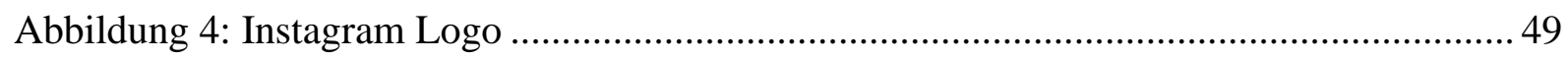

Abbildung 5: Screenshot Quickreactions (2020-06-28-11-47-49)........................................ 50

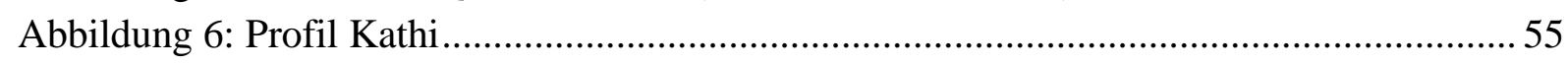

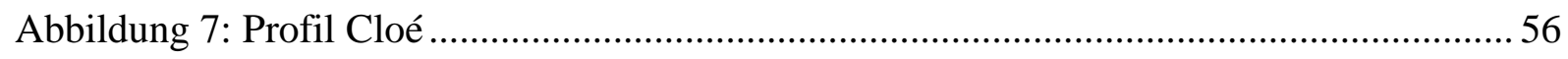

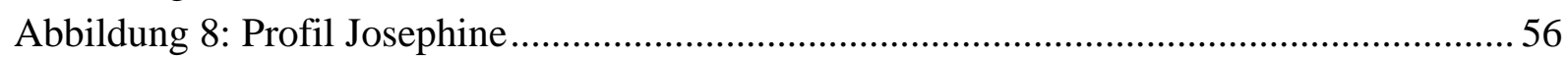

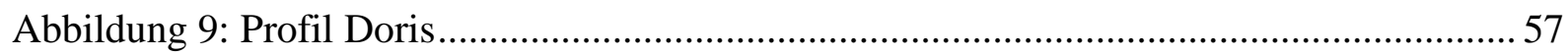

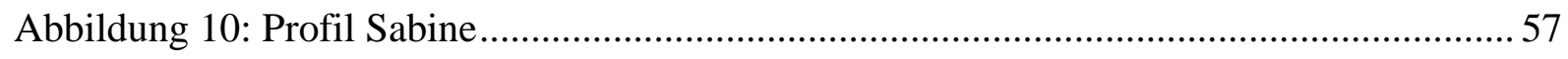

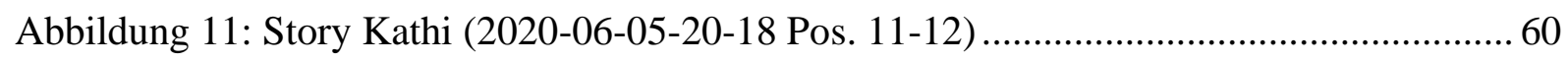

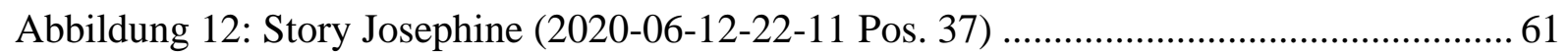

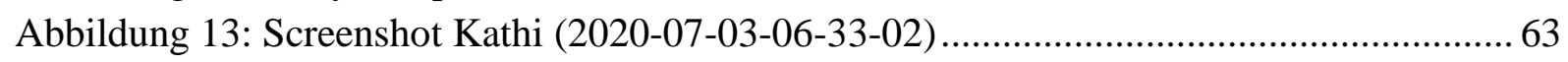

Abbildung 14: Bildunterschrift Post Kathi 02.07.2020 (Screenshot erstellt 06.10.2020)....... 63

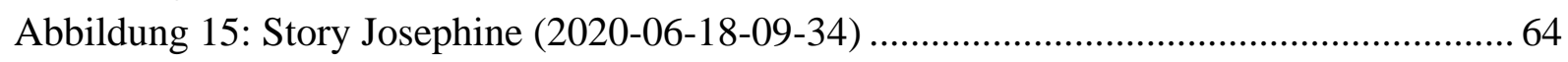

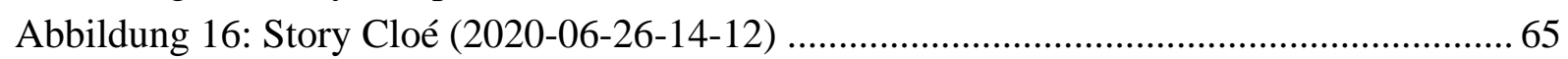

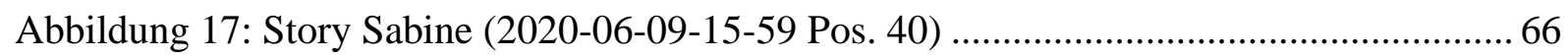

Abbildung 18: Sabine Post vom 30.08.2019 (Screenshot erstellt am 05.10.2020) ...................68

Abbildung 19: Doris Post (Screenshot erstellt am 18.09.2020) ............................................... 69

Abbildung 20: instarepeat Post (Screenshot erstellt am 31.08.2020) ....................................... 70

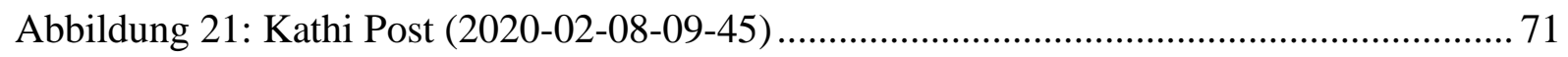

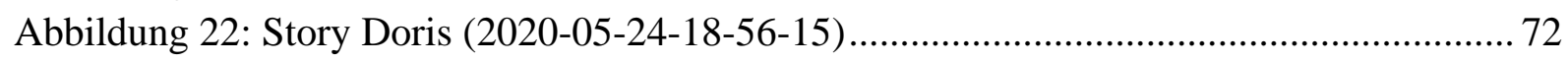

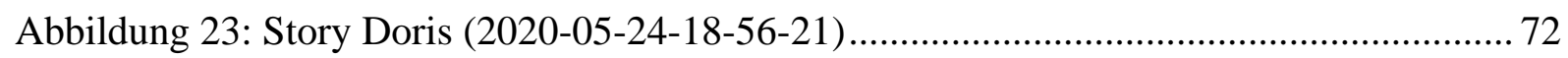

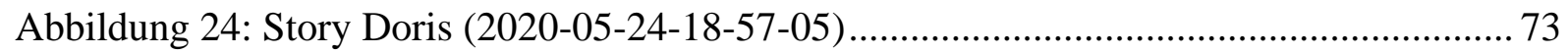

Abbildung 25: Ausschnitt Story Cloé (2020-06-29-11-06-37) .............................................. 74

Abbildung 26: Post Kathi 20.03.2019 (Screenshot erstellt am 02.08.2020) .......................... 75

Abbildung 27: Post Kathi 31.07.2020 (Screenshot erstellt am 02.08.2020) .......................... 75

Abbildung 28: Post Sabine 12.07.2020 (Screenshot erstellt 04.09.2020) ................................ 77

Abbildung 29: Fraktale Unterscheidung Selbstthematisierung (eigene Darstellung) ..............86 


\section{Eigenständigkeitserklärung}

Hiermit versichere ich, Magdalena Riedl (180416), dass ich diese Masterarbeit mit dem Thema: „Lifelogging auf Instagram - Digitale Selbstvermessung und -darstellung zwischen disruptiver Technologie und kulturellem Wandel“" selbstständig verfasst, keine anderen als die angegebenen Quellen und Hilfsmittel benutzt, sowie direkte und indirekte Zitate als solche kenntlich gemacht habe.

Jena, 20.10.2020

Ort, Datum

Unterschrift 